\title{
Applications of laser wakefield accelerator-based light sources
}

\author{
Félicie Albert \\ Lawrence Livermore National Laboratory, NIF and Photon Sciences, Livermore, CA 94550, USA \\ Alec G.R. Thomas \\ Department of Nuclear Engineering and Radiological Sciences and Department of Physics and \\ Center for Ultrafast Optical Science, University of Michigan, Ann Arbor, MI 48109 USA
}

\section{Contents}

1 Introduction $\quad 1$

2 Current performances of Laser wakefield accelerator-based light sources 2

2.1 Laser wakefield accelerators . . . . . . . . . . . . . . . 2

2.1.1 Scalings for maximum energy gain and accelerator length . . . . . . . 5

2.1.2 Injection into the wakefield . . . . . . . . . . . . . . . . 7

2.2 Betatron X-ray Radiation . . . . . . . . . . . . . . . . . 8

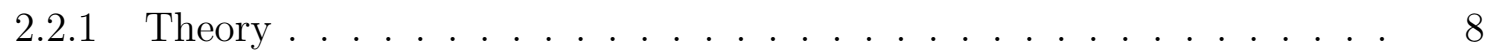

2.2.2 Experimental source properties . . . . . . . . . . . . . 10

2.2.3 Betatron as a diagnostic for LWFA electrons . . . . . . . . . . . . . 10

2.2.4 Source control and comparison with conventional synchrotrons . . . . 14

2.3 Compton Scattering . . . . . . . . . . . . . . . . . . . . . 14

2.3.1 Principle of Inverse Compton scattering with laser wakefield accelerators 14

2.3.2 Compton scattering experiments with laser wakefield accelerators . . 15

2.3.3 Comparison with Compton scattering from RF accelerators . . . . . 16

2.3 .4 Radiation Reaction . . . . . . . . . . . . . . . . . . 19

2.4 Undulator and XFEL radiation . . . . . . . . . . . . . . . . . . . . . . . . . . . . . . . . .

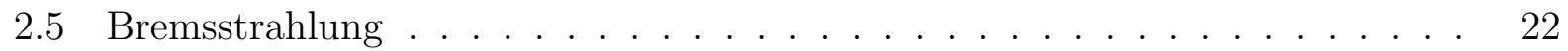

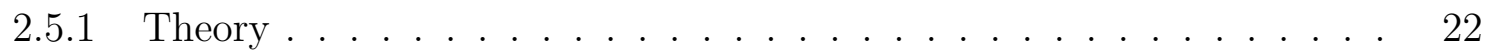

2.5.2 Bremsstrahlung produced by LWFA electrons . . . . . . . . . . . . 23

$2.6 \mathrm{THz}$ and coherent transition radiation . . . . . . . . . . . . . . 24 
3 Medical and biological applications $\quad 25$

3.1 Diagnostic radiology using LWFA X-ray sources . . . . . . . . . . . . . . 26

3.2 Nuclear medicine . . . . . . . . . . . . . . . . . . . . . . . . . . . . . . . . . . . . . 27

3.3 Biomedical applications of $\mathrm{THz}$ radiation . . . . . . . . . . . . . . . . . . . 29

4 Military, defense and industrial applications $\quad 29$

4.1 High resolution gamma-ray radiography . . . . . . . . . . . . . . . . . . . . . 29

4.2 Backscattered X-ray and gamma-ray inspection . . . . . . . . . . . . . . 30

4.3 Isotope-specific detection with nuclear resonance fluorescence . . . . . . . . . 31

4.4 Nuclear waste treatment and photo transmutation . . . . . . . . . . . . . 32

4.5 Energy storage: nuclear excitation by electron capture . . . . . . . . . . 34

4.6 Detection of explosives and drugs with $\mathrm{THz}$ spectroscopy . . . . . . . . . . . 35

4.7 Properties of semiconductors investigated with $\mathrm{THz}$ radiation . . . . . . . 35

5 Condensed matter and high energy density science 36

5.1 Condensed matter physics . . . . . . . . . . . . . . . . 36

5.2 Laser-driven shocks . . . . . . . . . . . . . . . . . . . . . . . . . 38

5.3 Electron-ion thermalization in warm dense matter . . . . . . . . . . . 38

5.4 Opacity in hot dense plasmas . . . . . . . . . . . . . . . . . . . . 39

5.5 Experimental techniques enabled by LWFA-driven sources . . . . . . . . . . 40

5.5.1 Radiography and X-ray phase contrast imaging . . . . . . . . . . . . 40

5.5 .2 X-ray absorption spectroscopy . . . . . . . . . . . . . . . . . . . 42

5.5.3 X-ray diffraction and scattering ............... 43

6 Conclusion and outlook $\quad 44$

7 Acknowledgements $\quad 46$ 


\begin{abstract}
Laser-wakefield accelerators (LWFAs) were proposed more than three decades ago, and while they promise to deliver compact, high energy particle accelerators, they will also provide the scientific community with novel light sources. In a LWFA, where an intense laser pulse focused onto a plasma forms an electromagnetic wave in its wake, electrons can be trapped and are now routinely accelerated to $\mathrm{GeV}$ energies. From terahertz radiation to gamma-rays, this article reviews light sources from relativistic electrons produced by LWFAs, and discusses their potential applications. Betatron motion, Compton scattering and undulators respectively produce X-rays or gamma-rays by oscillating relativistic electrons in the wakefield behind the laser pulse, a counterpropagating laser field, or a magnetic undulator. Other LWFA-based light sources include bremsstrahlung and terahertz radiation. We first evaluate the performance of each of these light sources, and compare them with more conventional approaches, including radio frequency accelerators or other laser-driven sources. We have then identified applications, which we discuss in details, in a broad range of fields: medical and biological applications, military, defense and industrial applications, and condensed matter and high energy density science.
\end{abstract}




\section{Introduction}

Modern particle accelerators use radio-frequency (RF) waves travelling in metal cavities to accelerate particles. These waves have a longitudinal electric field which propagates near the speed of light, $c$, allowing relativistic particles $(v \simeq c)$ to remain in the accelerating phase of the field. Ionisation of the walls at high voltages means they cannot support electric field gradients greater than $\sim 100 \mathrm{MVm}^{-1}$, though the operating limit is usually more like $\sim 10$ $\mathrm{MVm}^{-1}$. This limits the highest energies achievable through cost, as each GeV of energy requires $\sim 100 \mathrm{~m}$ of acceleration length.

One important offshoot of particle acceleration in synchrotrons was the use of the radiation generated by the accelerating particles for molecular crystallography, fluorescence studies, chemical analysis, medical imaging, and many other applications [1, 2]. A synchrotron producing X-rays requires more modest particle energies of a few $\mathrm{GeV}$, but this still means national laboratory scale facilities, which limits access. For example, the Diamond light source in the UK cost around $£ 300$ million to construct. Another source of $\mathrm{X}$-rays for science is the free-electron laser. In this scheme, a high energy electron bunch is propagated through an undulator - a periodic magnetic field structure - so that radiation is produced by the bunch in the forward direction. The effective 'lasing medium' in this case is the electron bunch, which interacts with the electromagnetic wave such that the resulting radiation is amplified over the propagation length. The free-electron laser LCLS at the SLAC national accelerator laboratory in the USA [3], the European XFEL at DESY in Germany, and SACLA at Spring-8 in Japan are examples of such facilities.

If the limiting factor on the scale of the accelerator is ionisation of the material, then an attractive alternative is to use a plasma. A plasma can support arbitrarily high electric fields, limited only by the obtainable charge density (and eventually, quantum limits). Longitudinal electric fields moving at the speed of light are supported in the form of electron plasma waves with relativistic phase velocity. Generating these relativistic phase velocity plasma waves requires a relativistic particle beam, including photons in the form of intense laser pulses. Current ultra-high-power laser systems capable of driving such waves are typically Ti:Sapphire based technologies with central wavelength $0.8 \mu \mathrm{m}$. The maximum electric field possible is therefore limited to $E_{\max }<1 \mathrm{TVm}^{-1}$ because there is a maximum plasma density that the light can propagate in. However, this is already $10,000 \times$ greater than the highest field of RF-based cavities, and therefore would allow substantial miniaturization of the accelerator; potentially a $\mathrm{km}$ scale facility may be realized on the $\mathrm{m}$ scale and as a consequence, the access of universities, research establishments and less developed nations to advanced photon sources may be increased dramatically. The $\sim$ few GeV sources needed for X-ray production realistically need only cm's of acceleration length, with the main bulk of the accelerator then being the laser itself rather than the accelerator.

In this review paper, we discuss the basic principles and properties of light sources from electrons accelerated in laser-driven plasmas, and we present their current and potential applications in medicine, industry and defense, and high energy density science. In Section 2, we briefly outline the physics of laser wakefield acceleration (we also refer the reader to a recent review paper on this subject [4]), and describe five different sources from laser wakefield accelerators: betatron radiation, Compton scattering, undulator and free electron laser

radiation, bremsstrahlung, and optical transition/terahertz radiation. While the objective 
of this paper is not to present a detailed theoretical study of such light sources (for this we refer the reader to recent reviews [5]), we give sufficient background in order to understand their current performances and possibilities for applications. The sources, described in more details in Section 2, are illustrated in Figure 1. The name "betatron radiation" or "betatron X-rays" has been adopted by the laser-plasma community to describe the radiation emitted by electrons performing betatron oscillations in the plasma wakefields, and hence in this paper we use the term in this context.

In Section 3 we review applications relevant to medicine, such as diagnostic radiology through imaging, radiotherapy and nuclear medicine. Then, in Section 4 we present applications for the industry and defense sectors, such as gamma-ray radiography, backscattered X-ray or gamma-ray inspection, isotope-specific detection, nuclear waste treatment and photofission, detection of explosives and drugs, and semiconductor imaging. In Section 5, we highlight applications for high energy density (HED) science, a growing field studying conditions found in extreme environments, such as fusion plasmas or planetary and solar interiors [6]. In particular, we discuss electron-ion equilibration mechanisms in these environments, shock physics, and opacity in hot, dense plasmas. Consequently, we present a few diagnostics techniques where light sources based on laser-plasma accelerators could offer a real alternative to conventional technology.

\section{Current performances of Laser wakefield accelerator- based light sources}

\subsection{Laser wakefield accelerators}

Laser plasma wakefield acceleration (LWFA) is a method of generating a plasma wave with relativistic phase velocity using an ultrashort pulse. It was first proposed in 1979 in a paper by T. Tajima and J.M. Dawson [7]. The rate of progress towards experimental demonstration of the various schemes has been determined by technological constraints. The coupling of laser momentum to the plasma is mediated by the ponderomotive quasi-force $F_{P}$, which arises due to the second-order motion of electrons in the intensity gradients of the light

pulse and can be expressed as $F_{P} \propto-\nabla \gamma$, where $\gamma=1 / \sqrt{1-\frac{v^{2}}{c^{2}}}$ is the electron relativistic factor [4]. To gain a reasonable electrostatic potential in the wake $\phi \sim \Phi_{P}$ on the order of the ponderomotive potential, the electron motion must be relativistic, which requires the product of intensity with wavelength squared to be $I \lambda^{2}>10^{17} \mathrm{Wcm}^{-2} \mu \mathrm{m}^{2}$. The gradient of the laser pulse must also be relatively short for the ponderomotive force to be significant in the longitudinal direction, with scale length $\sim c / \omega_{p}$, where $\omega_{p}=\sqrt{n_{e} e^{2} / m \epsilon_{0}}$ is the plasma frequency for a plasma of electron density $n_{e}$. Nd:Glass and $\mathrm{CO}_{2}$ Lasers in the early 1990's could achieve the necessary intensities, but were long pulses and were therefore not capable of coupling to the plasma efficiently.

The advent of chirped pulse amplification [8] allowed significantly shorter (ps), more pow$\operatorname{erful}(P>$ TW $)$ laser pulses to be produced. These pulses could have $I \lambda^{2} \gg 10^{18} \mathrm{Wcm}^{-2} \mu \mathrm{m}^{2}$ if focused to a small spot, but for the longer interaction lengths required for wakefield acceleration, the laser experienced strong non-linear optical self-focusing. This meant that for such 


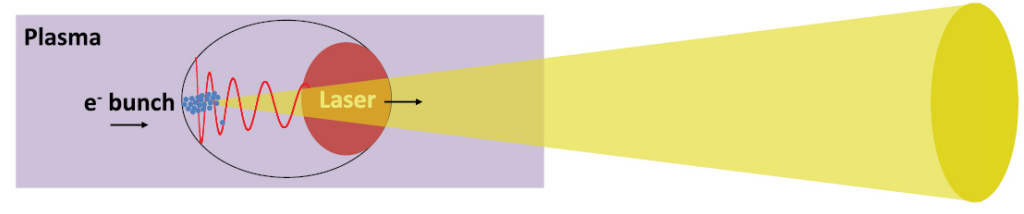

Betatron

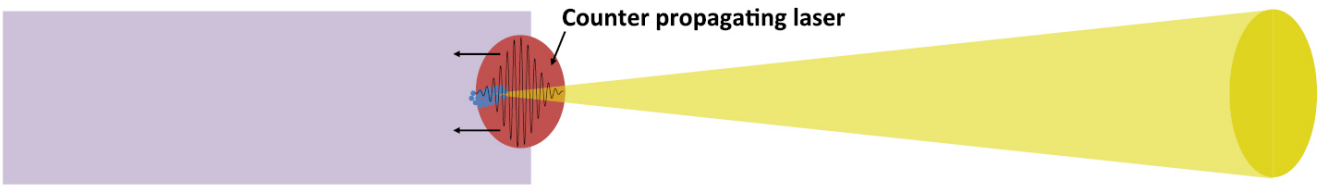

Compton
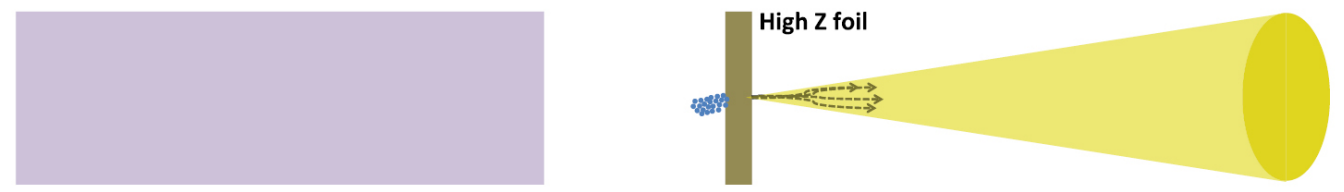

Bremsstrahlung
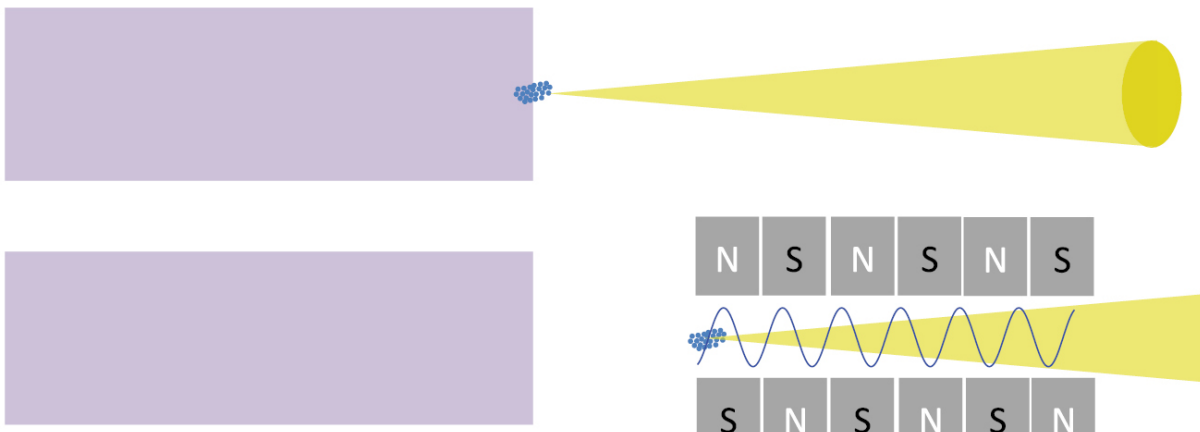

Terahertz/OTR

Figure 1: Illustration of the five principal LWFA-driven light sources described in this paper. Betatron radiation is produced by electrons accelerated and wiggled by the longitudinal and transverse wakefields at the back of the laser pulse. Compton scattered light is emitted when LWFA electrons are wiggled by a transverse laser field, either reflected from a plasma mirror or from a secondary pulse. Bremsstrahlung is produced when relativistic electrons interact with a high- $\mathrm{Z}$, solid material and $\mathrm{THz}$ /optical transition radiation is emitted when the LWFA electrons traverse the plasma/vacuum or vacuum/solid interface with a dielectric constant discontinuity. Undulator and FEL radiation is produced when LWFA electrons are wiggled by external magnetic field, like in a synchrotron or FEL. 
lasers, the duration must be relatively long, $\tau>1 / \omega_{p}$, for densities where self-focusing of the pulse could overcome diffraction. For efficient wakefield generation, the duration of the laser pulse should ideally be less than the plasma period $2 \pi c / \omega_{p}$, which for atmospheric density Hydrogen plasma is of order 10-100 fs. In early experiments, such lasers were not available, but electrons were still accelerated by self-modulated LWFA $[9,10,11,12]$. If a pulse longer than $2 \pi c / \omega_{p}$ is used, but having sufficiently high intensity, relativistic self-phase modulation of the pulse can result [13]. Frequency shifts induced by a seed plasma wave results in a variation in group velocity along the pulse, causing modulation of the pulse envelope at the plasma period, which increases the ponderomotive force and thereby reinforces the original perturbation.

The self-modulated laser wakefield accelerator (SM-LWFA) [14, 15, 10, 16, 17] occurs when the pulse is of sufficient power that non-linear optical effects dominate the pulse propagation, but the pulse is very long compared with $1 / \omega_{p}$. The result is that the pulse is longitudinally and transversely modulated into a train of pulses which then drive a wake through the ponderomotive force. The advantage over the beatwave scheme is that resonance is enforced by the self-modulation. This can result in large amplitude wave growth $[9,18]$, and beyond the wavebreaking limit, self-trapping of electrons from the background plasma. The first experimental evidence for electron trapping and acceleration to $44 \mathrm{MeV}$ in a SM-LWFA was reported by Modena et al [11]. Other experiments subsequently reported self-trapping in a SM-LWFA [19, 20, 12].

A related mechanism to SM-LWFA is "direct laser acceleration" [21, 22, 23, 24], which occurs when the pulse is long but sufficiently intense to generate a channel void of electrons with strong radial fields. When electrons experience the combination of channel and laser fields, coupled oscillator behaviour results, with characteristic chaotic orbits and large forward directed energies achievable. This can be a source of very large currents of electrons and potentially a high flux X-ray source [25]. In the case of self-modulated LWFA, because of the long duration, the electrons interact with both laser and plasma fields in a similar manner, leading to chaotic orbits and therefore exponential particle energy distributions result.

An important milestone in experimental LWFA studies was the first observations of self-trapped quasi-monoenergetic beams of electrons [26, 27, 28, 29]. Previous experiments on laser wakefield acceleration had reported electron beams with a maxwellian electron spectrum, which was the result of performing the experiments at necessarily high densities. Due to the slower group velocity of the laser, the electrons eventually outrun the pulse and gain a large spread in energy as they reach the decelerating part of the potential. The advent of $<50 \mathrm{fs},>10 \mathrm{TW}$ lasers allowed the pulse to be focused to a larger spot, but still with $I \lambda^{2} \gg 10^{18} \mathrm{Wcm}^{-2} \mu \mathrm{m}^{2}$. This allowed self-trapping to occur at a lower density than previously, so that the dephasing limit was not exceeded and monoenergetic electron beams could be extracted [30]. However, to obtain a single monoenergetic spike the laser intensity should be sufficient that only a single wave period trails the pulse, due to high amplitude wave oscillations in three-dimensions [31].

Following experimental results generating $~ 100 \mathrm{MeV}$ electrons, LWFAs demonstrated a rapid increase in maximum energy, with the $\mathrm{GeV}$ threshold being quickly reached [32, 33, 34, 35, 36]. Electrons approaching $5 \mathrm{GeV}$ have been recently been measured [37]. Since such energies are already comparable to those in 3rd generation synchrotron facilities, the use of wakefield accelerators for photon light source applications is a near-term prospect. 


\subsubsection{Scalings for maximum energy gain and accelerator length}

The longitudinal electric field of a wakefield can be used to accelerate electrons or positrons, but there are physical limitations to the maximum energy that can be achieved. Deriving simple scalings for both the maximum energy gain and the accelerator length can provide important guidelines to be able to understand potential limitations and to advance laserplasma based accelerator designs.

A wakefield in a uniform plasma of electron number density $n_{e}$ has a phase velocity $v_{p}$ dictated (to first order) by the group velocity $v_{g}$ of the driver laser pulse, with central wavelength $\lambda_{0}$. This is because a laser pulse exerts a time averaged force on the plasma electrons, known as the ponderomotive force, that is proportional to the gradient of the laser envelope. The strength of the laser field $\mathcal{E}$ is characterized by the work done by this field over a wavelength relative to the electron rest mass energy, $a_{0}=e \mathcal{E} \lambda_{0} /\left(2 \pi m_{e} c^{2}\right)$. Perturbed plasma electrons will oscillate at a frequency $\omega_{p}=\sqrt{e^{2} n_{e} / m_{e} \epsilon_{0}}$ due to the electric field resulting from the charge separation relative to the ion background. In the $1 \mathrm{D}$ linear regime, the group velocity is $v_{g}=\sqrt{1-n_{e} / n_{c}}$, where $n_{c}=4 \pi^{2} m_{e} c^{2} \epsilon_{0} / e^{2} \lambda_{0}^{2}$ is the electron number density for which the light is no longer able to propagate in the plasma. Note that in 3D nonlinear regime, the laser group velocity is not easily defined. For a sufficiently low density plasma, a relativistic particle initiated in an accelerating phase of the electric field will gain energy as it stays in phase with the wave for long distances.

A relativistic particle with $v \simeq c$ will eventually outrun the accelerating phase of the plasma wave, which in the linear regime has a phase velocity equal to $v_{g}<c$. As a consequence, there is a maximum energy that can be extracted from the wakefield for a given laser wavelength and plasma density. This phenomenon is known as dephasing. It can be shown that in the $1 \mathrm{D}$ linear regime, this maximum energy is $E_{\max } \simeq\left(n_{c} / n_{e}\right) m_{e} c^{2}[7,4]$. The distance over which this dephasing occurs is $L_{\text {deph }} \simeq\left(n_{c} / n_{e}\right) \lambda_{p} / 2 \propto\left(n_{c} / n_{e}\right)^{3 / 2}$. In the 3D nonlinear regime $[38,39,40]$, it has been shown that the transverse size of the structure is dictated by self-focusing of the laser pulse and scales as $1 / \omega_{p}$. This means that to go to lower plasma density to achieve higher energy electrons, as these scalings suggest, the laser pulse energy must also increase. This a technical challenge, since generating high energy, ultrashort pulses is limited by optical component damage thresholds. Under these conditions and at higher intensities, the wake field shape resembles that of a spherical "bubble" of radius $r_{b} \simeq 2 \sqrt{a_{0}} c / \omega_{p}$ and this regime is consequently often referred to as the "bubble regime" of LWFA [38]. An illustration of a typical laser wakefield accelerator in this regime is shown in Figure 2.

To find scalings for the accelerator length and maximum energy achievable, let us assume that the wake, regardless of the details of the plasma wakefield structure, has a potential $\phi(\xi, \vec{r})$, where $\xi=z-v_{g} t$ is the wake phase coordinate traveling with velocity $v_{g}$ (this implies a non-evolving wakefield). Then, in the rest frame (denoted by primed quantities) of the wakefield, the potential will be $\phi^{\prime}=\gamma_{p}\left(\phi-v_{g} A_{z}\right)=\gamma_{p} \phi$ in the Lorenz gauge, where $\gamma_{p}$ is the Lorentz factor associated with the boost. We assume that the difference $c-v_{g}$ is proportional to the plasma density so that $\gamma_{p}=\sqrt{\eta n_{c} / n_{e}}$, where $\eta$ is some constant (for linear dispersion this is exact, with $\eta=1$ ). The maximum energy gain of the particle in this frame is $\Delta T^{\prime}=e \Delta \phi^{\prime}$, where $\Delta \phi^{\prime}$ is the difference between the maximum and minimum wake potential. 


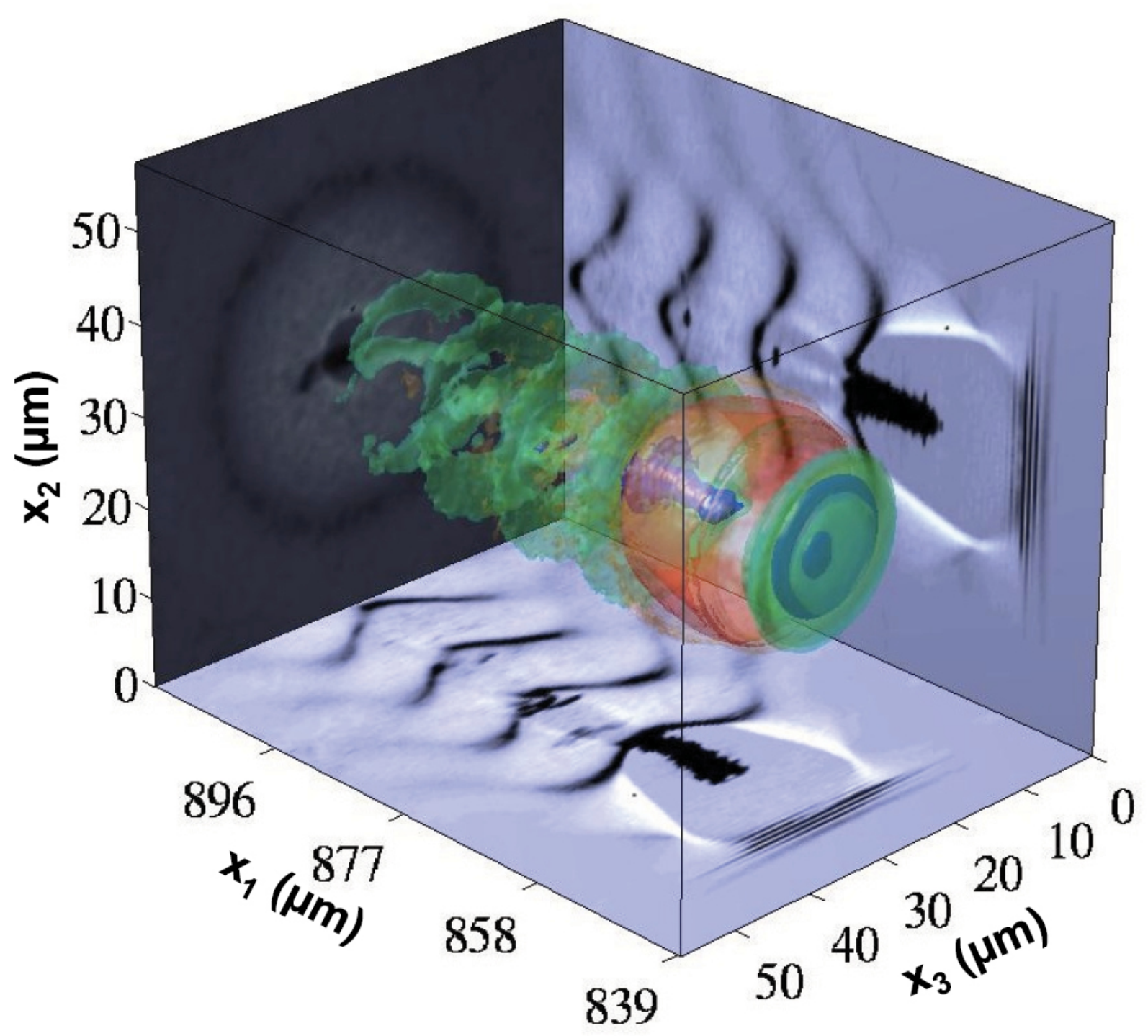

Figure 2: Illustration of laser wakefield acceleration in the bubble regime: isosurface plots calculated from the 3D particle in cell code OSIRIS 2.0, in a box moving at the speed of light, taken after $0.84 \mathrm{~mm}$ propagation. It models the propagation of a gaussian laser pulse with pulse duration $29 \mathrm{fs}$ FWHM, beam waist $w_{0}=10 \mu \mathrm{m}$, and normalized vector potential of $a_{0}=4$ in a Helium plasma with a fully ionized electron density of $0.005 n_{c}$. Side panels display slices through the center of the box. The isosurfaces correspond to: blue, $10 \%$ and green, $2 \%$ of minimum and red, $25 \%$ and orange, $50 \%$ of maximum current. 
We can transform the energy gained by an electron in this static potential back to the laboratory frame using the transform $\Delta T=\gamma_{p}\left(\Delta T^{\prime}+v_{g} \Delta p^{\prime}\right) \approx 2 \gamma_{p} \Delta T^{\prime}$, where we have used the limit $\gamma_{p} \Delta \phi \gg 1$ so that $\Delta p^{\prime} c \approx \Delta T^{\prime}$. Hence, the energy gain in the laboratory frame is $\Delta T=2 \gamma_{p}^{2} e \Delta \phi$, or

$$
\Delta T \simeq C_{1}\left(\frac{n_{c}}{n_{e}}\right) m_{e} c^{2},
$$

where $C_{1}=2 \eta\left(e \Delta \phi / m c^{2}\right)$ is a scaling factor that depends on the geometry and amplitude of the wakefield. Since by dimensional analysis the longitudinal electric field has a magnitude of order $E_{0}=m c \omega_{p} / e$, the length over which this acceleration occurs is

$$
L_{\text {deph }} \simeq C_{2}\left(\frac{n_{c}}{n_{e}}\right)^{3 / 2} \lambda_{0} .
$$

where $C_{2}=C_{1} / 2 \pi \alpha$ and the electric field averaged between the maximum and minimum potentials is $\left\langle E_{z}\right\rangle=\alpha E_{0}$. Determining the exact values of the scaling constants for 3D nonlinear plasma waves requires numerical simulation. Note that since a non-evolving wakefield has been assumed, these strictly only apply for uniform plasma density and a non-evolving laser pulse and under different conditions, different scalings will apply.

In Ref. [40], the 3D scalings were studied using a mixture of analysis and large scale numerical simulations. These result in

$$
\begin{aligned}
\Delta E & \simeq \frac{2 a_{0}}{3}\left(\frac{n_{c}}{n_{e}}\right) m_{e} c^{2}, \\
L_{\text {deph }} & \simeq \frac{2 \sqrt{a_{0}}}{3 \pi}\left(\frac{n_{c}}{n_{e}}\right)^{3 / 2} \lambda_{0} .
\end{aligned}
$$

These scalings are in agreement with experimental results [41, 42].

\subsubsection{Injection into the wakefield}

One very important consideration in LWFAs is the process for injecting the electrons into the correct phase of the wake to be accelerated. Electrons can be injected externally, but obtaining a sufficiently short duration electron bunch is nontrivial. The electron bunch to be injected should ideally be temporally short compared with $1 / \omega_{p}$, so that the electrons experience similar magnitude electric field, and therefore uniform acceleration, although it has been suggested that the wakefield can naturally bunch an electron beam [43]. When the wakefield amplitude is sufficiently large that the oscillating electron velocities in the plasma fluid oscillation exceed the phase velocity of the wave, they will be self-trapped under certain conditions $[44,45,46,30,47]$. This generally requires $I \lambda^{2} \gg 10^{18} \mathrm{Wcm}^{-2} \mu \mathrm{m}^{2}$. The selfinjection of electrons can be increased in effectiveness by having a non-static bubble [48] or a thermal plasma [49]. Other methods are using a second laser to perturb electron orbits and allow them to cross the separatrix (between trapped and untapped orbits) in the phase space diagram. For example, all optical injection $[50,51,52,53,29,54]$, demonstrates the most control of the phase space distribution [55, 56, 57], while the use of density transitions [58], has demonstrated outstanding stability in experiments [59,60,61, 62, 63]. 
One notable mechanism is the use of ionization of inner shell electrons near the peak of the laser intensity to perturb electron orbits from the fluid motion [64, 65, 66, 34, 67, 68, 69, 70]. This requires adding a small amount of higher- $Z$ gas to the gas used for acceleration. This technique has shown great stability in gas cells [71], but also has a great outlook for control of the electron bunch phase space distribution, since the ionization process can be decoupled from the drive laser pulse by the introduction of a second laser pulse [67] and would be very useful for plasma based light sources.

\subsection{Betatron X-ray Radiation}

Simultaneous to laser wakefield acceleration, electrons can also undergo transverse oscillations if they are trapped away from the laser and plasma wave longitudinal axis. This motion of relativistic electrons, called betatron oscillations, produces, as in a synchrotron, a collimated beam of broadband X-rays with a great potential for applications. The first observation of betatron radiation was reported in a beam-driven wake at SLAC [72], where $28.5 \mathrm{GeV}$ electrons wiggled in a $1.4 \mathrm{~m}$ Lithium plasma to produce $\sim 10^{6}$ photons around $14.5 \mathrm{keV}$, which was followed by its characterization in a laser-driven wake [73], where $\sim 10^{8}$ photons were produced in the 1-10 keV energy range. The research on betatron X-ray source characterization and development in LWFAs has been very prolific over the past decade, and there are several excellent theoretical and experimental reviews of betatron X-ray radiation in the literature $[74,5]$. Here we describe some of the most relevant properties of the source for applications.

\subsubsection{Theory}

In the blowout regime, the equation of motion for an electron performing betatron oscillations while being accelerated by the longitudinal fields of the wake can be described by simplified expressions assuming a spherical ion cavity [75, 76]. In this structure, the electric field in the direction of propagation is given by $E_{z}=m_{e} \omega_{p}^{2} \xi / 2 e$, where $\xi=z-v_{g} t$, which has a maximum amplitude at the edge of the bubble at $r_{b}=2 \sqrt{a_{0}} c / \omega_{p}$ of $\left|E_{z}\right|_{\max }=\sqrt{a_{0}} m_{e} c \omega_{p} / e$, is zero at the center of the bubble where $\xi=0$ and therefore the average field strength is $\left\langle E_{z}\right\rangle=\left|E_{z}\right|_{\max }=\left(\sqrt{a_{0}} / 2\right) m_{e} c \omega_{p} / e$. The transverse electric fields are $E_{x}=m_{e} \omega_{p}^{2} x / 4 e$ and $E_{y}=m_{e} \omega_{p}^{2} y / 4 e$ and the magnetic field is only azimuthal, given by the components $B_{x}=m_{e} \omega_{p}^{2} y / 4 e$ and $B_{y}=-m_{e} \omega_{p}^{2} x / 4 e$. Since the components of the force are $F_{z}=-e E_{z}$, $F_{x}=-e\left(E_{x}-v_{z} B_{y}\right)$ and $F_{y}=-e\left(E_{y}+v_{z} B_{x}\right)$, and taking $v_{z} \simeq c$, the force on an electron within the bubble can be written in the compact form

$$
\frac{d \vec{p}}{d t}=-\frac{1}{2} m_{e} \omega_{p}^{2} \vec{s}
$$

where $\vec{s}=(x, y, \xi)=\vec{r}-v_{g} t \hat{z}$ are the coordinates comoving with the bubble at velocity $v_{g}$. As shown in [75], because the longitudinal momentum is slowly varying (the equation of motion can be integrated to obtain $p_{z} \simeq \gamma_{p}^{2} a_{0} m_{e} c\left(1-\xi^{2} / r_{b}^{2}\right)$, for $\left.v_{z} \simeq c\right)$, the transverse equations of motion can be treated as a slowly varying oscillator, with betatron frequency 
$\omega_{b}=\omega_{p} / \sqrt{2 p_{z}}$, and WKB solutions:

$$
p_{x}=\frac{p_{x 0}}{\sqrt{p_{z}(\xi)}} \cos \left[\int \omega_{b}(\xi) d \xi\right] .
$$

From the position and momentum of the electron, it is possible to calculate the intensity emitted by the particle per unit frequency $d \omega$ and solid angle $d \Omega$, derived by Jackson [77] as:

$$
\frac{d^{2} I}{d \Omega d \omega}=\frac{e^{2} \omega^{2}}{4 \pi c}\left|\int_{-\infty}^{\infty} \vec{n} \times(\vec{n} \times \vec{\beta}) e^{i \omega\left(t-\frac{\vec{n} \cdot \vec{r}}{c}\right)} d t\right|^{2},
$$

where $\vec{n}$ is the vector corresponding to the direction of observation and $\beta=v / c$ the normalized electron velocity. Equation 7 can be simplified if the electrons undergo very strong oscillations, similarly to a synchrotron wiggler. It means that the wiggler parameter, $a_{\beta}=\gamma k_{\beta} r_{\beta}$ [74] is larger than unity. Here, $\gamma$ is the electron relativistic factor, $r_{\beta}$ the oscillation radius, and $k_{\beta}=k_{p} /(2 \gamma)^{1 / 2}$. This asymptotic limit describes the spectrum emitted by an electron, as observed at an angle $\theta$ from the plane in which the particle oscillates [77, 74]:

$$
\frac{d^{2} I}{d \Omega d \omega} \propto \frac{\gamma^{2} \xi^{2}}{1+\gamma^{2} \theta^{2}}\left[K_{2 / 3}^{2}(\xi)+\frac{\gamma^{2} \theta^{2}}{1+\gamma^{2} \theta^{2}} K_{1 / 3}^{2}(\xi)\right],
$$

where $K_{2 / 3}$ and $K_{1 / 3}$ are modified Bessel functions. Here, $\xi=\frac{\omega}{\omega_{c}}\left(1+\gamma^{2} \theta^{2}\right)^{3 / 2}$, where the critical frequency $\omega_{c} \simeq 3 a_{\beta} \gamma^{2} \omega_{\beta}$ and $\omega_{\beta}=\omega_{p} /(2 \gamma)^{1 / 2}$ is the betatron frequency. For $\theta=0$, the function $\xi^{2} K_{2 / 3}^{2}(\xi)$ peaks at $\xi \sim 1 / 2\left(\omega \sim 0.5 \omega_{c}\right)$ [74]. The spectrum, integrated over the full spatial extent of the betatron beam, is also derived from Equation 7:

$$
\frac{d I}{d \omega}=\sqrt{3} \frac{e^{2}}{c} \gamma \frac{\omega}{\omega_{c}} \int_{\frac{\omega}{\omega_{c}}}^{\infty} K_{5 / 3}(x) d x,
$$

where $K_{5 / 3}$ is another modified Bessel function. Equation 8 peaks at $\omega \sim 0.45 \omega_{c}$ for $\theta=0$ while Equation 9 peaks at $\omega \sim 0.3 \omega_{c}$.

The calculation of betatron radiation spectra or beam profiles has been widely used in conjunction with single electron models to explain the shape of the beam profile [78] or the radiation spectrum $[79,80]$. Several analytical studies of the radiation emitted by electrons oscillating in an ion channel have been benchmarked against Particle In Cell (PIC) simulations $[81,82,83]$. However, the above methods assume electron trajectories with specific initial conditions (injection radius or angular momentum) without taking into account the full description of the interaction itself. Scaling laws using electron trajectories calculated in a Lorentz boosted frame with simplified fields attempting to remove this assumption are also in reasonable agreement with experiments [46]. Additionally, betatron oscillations do not occur in a unique plane [84], and electrons trajectories can be influenced by the presence of the laser field in the wake [85, 82], especially after they dephase. PIC simulations can now be post-processed to track electron trajectories and calculate their radiated spectrum and power for very accurate results $[86,87,88]$. 


\subsubsection{Experimental source properties}

Early observation of betatron radiation was in a beam-driven plasma wakefield accelerator [72]. Some work has been done in the direct laser acceleration regime with a picosecond laser system [25], where conversion efficiencies (from electrons to X-rays) of up to $5 \%$ and X-rays up to $50 \mathrm{keV}$ have been reported, but data are still lacking in this regime.

Following its first observation in a laser-driven wake [73], most of the experimental work on betatron radiation has been done in the blowout regime of LWFA for laser energies between 1-10 joules and pulse durations of typically less than 100 fs. Betatron X-ray properties, produced at a number of facilities worldwide [73, 89, 80, 84, 36, 90, 91], are described in Table 1 , along with the laser and plasma parameters used in the experiments. Figure 4 summarizes the peak brightness of these sources and Figure 3 shows some of its most notable features. In this regime, the highest betatron energies to date from the blowout regime (peaking at $\sim 20$ $\mathrm{keV}$ ) have been reported from $2 \mathrm{GeV}$ electron beams accelerated at the Texas Petawatt Laser [36]. Betatron spectra peaked at $150 \mathrm{keV}$ produced by $700 \mathrm{MeV}$ electron beams have been reported in a slightly different regime at the Astra-Gemini laser facility [92]. These higher than usual photon energies are reported as being due to the fact that electron oscillation amplitudes are resonantly enhanced by their interaction with the back of the laser pulse [85].

Although a direct measurement of the source duration has yet to be done, PIC simulations suggest that it is on the order of or less than the laser pulse width, and the ultrafast nature of the source was demonstrated by detecting nonthermal melting in InSb [93].

\subsubsection{Betatron as a diagnostic for LWFA electrons}

For the past ten years, betatron radiation has been used as a compelling diagnostic of LWFAs, because the X-rays produced directly depend on the electron trajectories. While the electron energy can be measured with a magnetic spectrometer, and the electron plasma density with interferometry, there is no direct technique to measure the oscillation radii of the electron trajectories. All the following methods consistently agree with oscillation radii of $0.5-5 \mu \mathrm{m}$. The spectrum of the source, measured with filters and crystal spectrometers [79], or with single photon counting techniques [80, 95] is a good indicator of the electron injection radius $r_{0}$ into the wake. In this case, the radiation spectrum is calculated from electron trajectories using Equation 5, where $n_{e}$ and $\gamma$ are known, and where $r_{0}$ is left as a free parameter until the calculated spectrum matches the measured spectrum. The beam profile, usually measured on large indirect detection CCD cameras or image plates, has been used to retrieve the injection radius, but also the initial angular momentum [78, 100], the anisotropy of the oscillations [84, 101], or the nature (transverse or longitudinal) of electron self-injection into the LWFA [102]. The X-ray source size has been directly measured with Fresnel diffraction techniques [103, 89, 104]. A clean edge (preferentially an opaque, cleaved diffraction crystal) is placed in half of the beam, close (a few $10 \mathrm{~cm}$ ) to the source, and its shadow is cast on a detector further downstream (a few meters) to ensure a good resolution. The profile of this shadow is fitted with Fresnel diffraction integrals to retrieve the only unknown parameter, the source size. A similar imaging technique can be used to estimate the longitudinal extent of the source [105], typically a few 100 microns for TW laser systems. 


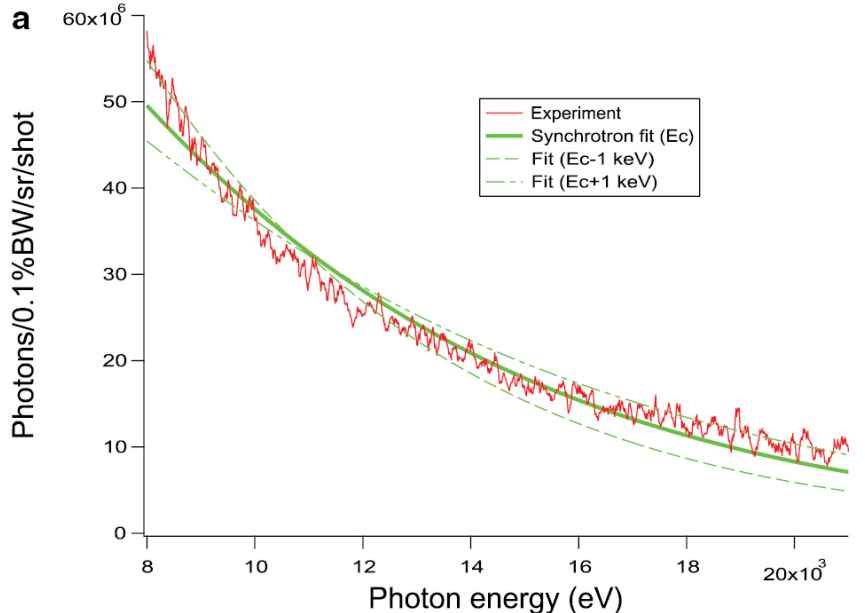

C

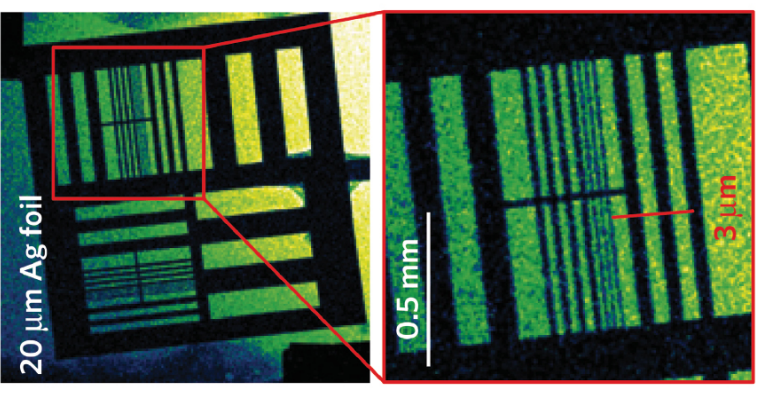

b

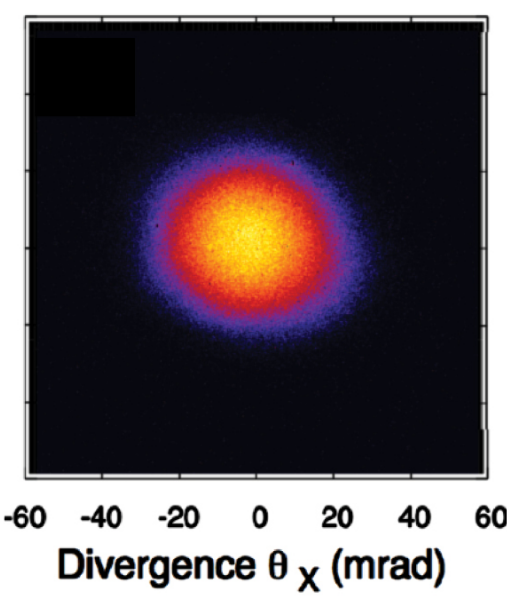

d

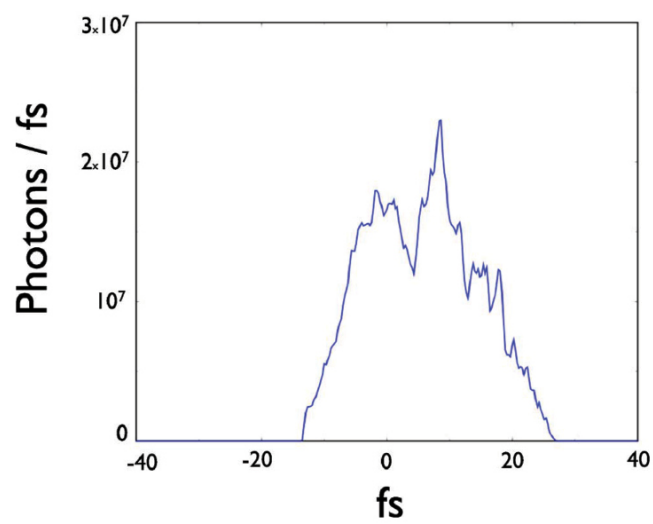

Figure 3: Notable features of betatron X-ray radiation. (a) Experimental synchrotron-like X-ray spectrum with $5.6 \mathrm{keV}$ critical energy (from Fourmaux et al [90]), (b) beam profile for photon energies $>2 \mathrm{keV}$ (from Ta Phuoc et al [78]), (c) radiographic image of a resolution target, demonstrating the micrometer size of the source (from Kneip et al [89]), and (d) pulse duration calculated from a PIC simulation (from Ta Phuoc et al [93]) 
Table 1: Betatron X-ray source and corresponding laser and plasma parameters obtained at a number of facilities worldwide

\begin{tabular}{|l|l|l|l|l|l|l|l|l|}
\hline $\begin{array}{l}\text { Laser fa- } \\
\text { cility }\end{array}$ & $\begin{array}{l}\text { Pulse } \\
\text { energy } \\
(\mathbf{J})\end{array}$ & $\begin{array}{l}\text { Pulse } \\
\text { dura- } \\
\text { tion } \\
(\mathbf{f s})\end{array}$ & $\begin{array}{l}\text { Spot } \\
\text { size } \\
(\mu \mathbf{m})\end{array}$ & $\mathbf{a}_{\mathbf{0}}$ & $\begin{array}{l}\text { Electron } \\
\text { density } \\
\left(\mathbf{c m}^{-3}\right)\end{array}$ & Photons & $\begin{array}{l}\text { Energy } \\
\text { at } \\
\text { in- } \\
\text { tensity } \\
\text { peak } \\
\mathbf{( k e V )}\end{array}$ & Year \\
\hline $\begin{array}{l}\text { Salle } \\
\text { Jaune }\end{array}$ & 1 & 30 & 18 & 1.2 & $8 \times 10^{18}$ & $10^{8}$ & 2 & $2004[73]$ \\
\hline $\begin{array}{l}\text { Astra- } \\
\text { Gemini }\end{array}$ & $10-15$ & $45-55$ & 20 & $2.5-3.5$ & $\begin{array}{l}3-10 \times \\
10^{18}\end{array}$ & $10^{9}$ & 33 & $2014[94]$ \\
\hline Hercules & 2.3 & 32 & 11.2 & 4.7 & $\begin{array}{l}4-22 \times \\
10^{18}\end{array}$ & $10^{6}-10^{8}$ & 10 & $2010[89]$ \\
\hline T-REX & 1.3 & 24 & 28 & 1 & $\begin{array}{l}4-10 \times \\
10^{18}\end{array}$ & $N . A$. & 4 & $2012[80]$ \\
\hline Callisto & $4-8$ & 60 & 12 & 2 & $5 \times 10^{18}$ & $10^{8}$ & 8 & $2013[84]$ \\
\hline $\begin{array}{l}\text { Texas } \\
\text { Petawatt }\end{array}$ & 170 & 150 & 60 & 2 & $2 \times 10^{17}$ & $10^{8}-10^{9}$ & 25 & $2013[36]$ \\
\hline ALLS & 2.5 & 30 & 24 & 1.2 & $5.4 \times 10^{18}$ & $3.6 \times 10^{7}$ & $2-3$ & $2013[90]$ \\
\hline JETI & 0.73 & 27 & 12 & 1.9 & $\begin{array}{l}2-20 \times \\
10^{18}\end{array}$ & $5 \times 10^{8}$ & 6 & $\begin{array}{l}2013 \\
95]\end{array}$ \\
\hline Lund & 0.65 & 40 & 19.7 & 1.6 & $\begin{array}{l}8-15 \times \\
10^{18}\end{array}$ & $10^{9}$ & 2 & $2013[96]$ \\
\hline XLII & 0.73 & 27 & 12 & 1.9 & $8 \times 10^{18}$ & $5 \times 10^{8}$ & $<0.5$ & $2013[97]$ \\
\hline ATLAS & 1.6 & 28 & 22 & 2.1 & $\begin{array}{l}8-11 \times \\
10^{18}\end{array}$ & $1.6 \times 10^{7}$ & 4.9 & $2013[98]$ \\
\hline Vulcan & 0.73 & 27 & 12 & 1.9 & $\begin{array}{l}2-20 \times \\
10^{18}\end{array}$ & $5 \times 10^{8}$ & 18 & $2013[25]$ \\
\hline
\end{tabular}




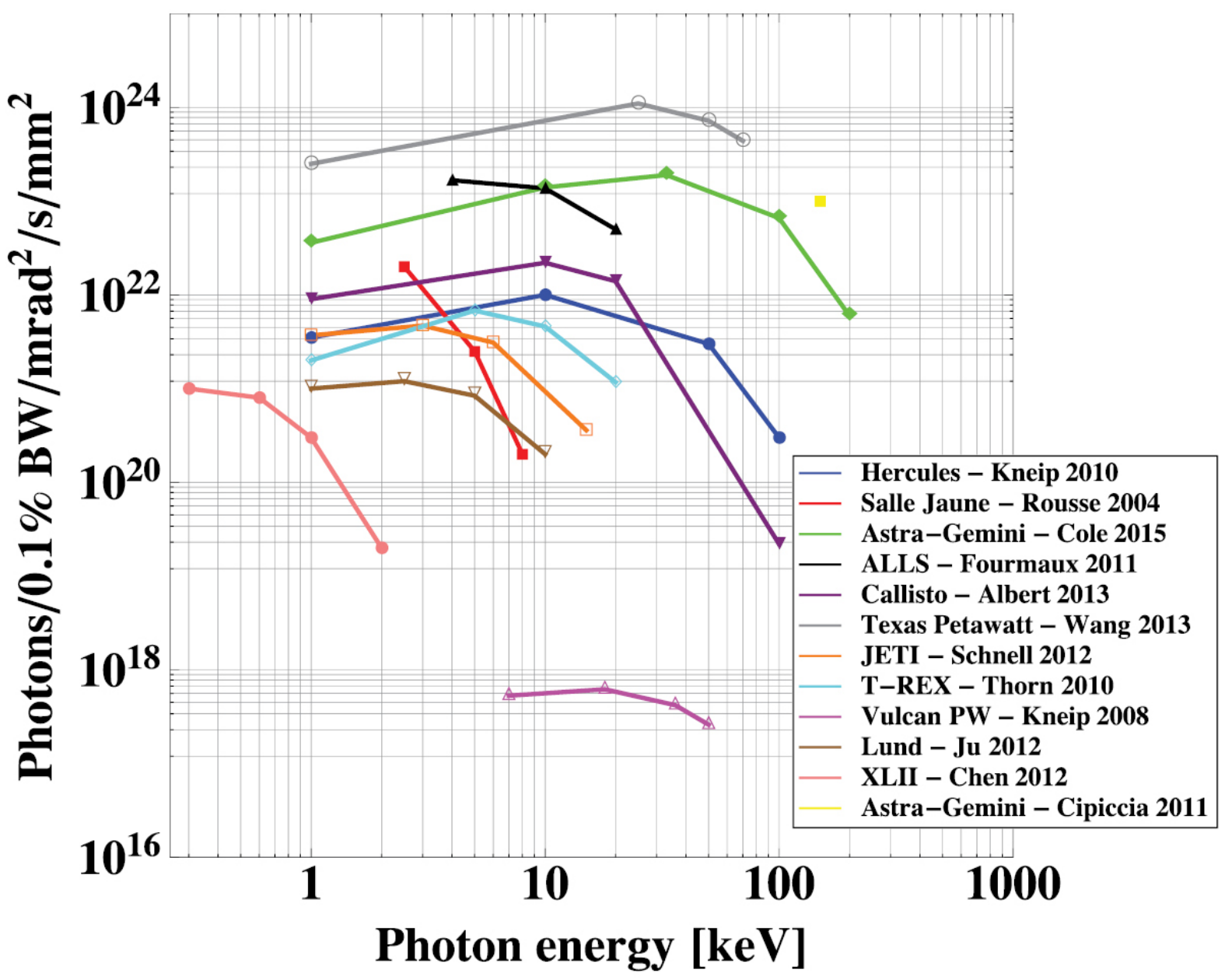

Figure 4: Peak brightness of betatron $\mathrm{X}$-ray sources produced at the facilities listed in Table 1. The publications of Rousse [73], Kneip 2010 [89], Kneip 2008 [25], Ju [96], Cipiccia [92], Schnell [91], Wenz [98] and Chen [97] directly provide this quantity, which is reported on the figure. We estimated the peak brightness based on the source parameters given in the other references: Cole [94] gives the average brightness and flux, Foumaux [90] the number of photons per unit solid angle and spectral bandwidth, Plateau/Thorn [80, 99] and Albert [84] the total number of photons, the divergence, spectrum and upper source size limit, Wang [36] the total number of photons, the divergence and spectrum (we assumed a micron-scale source size). The lower brightness quoted by Kneip et al 2008 [25] is due to the fact that the betatron X-rays where produced with longer (ps) petawatt-class laser pulses. The experiment of Chen et al [97] was performed using cluster argon targets of higher densities, in the selfmodulated wakefield acceleration regime. The experiments of Plateau [80,99] and Schnell [91] used betatron radiation as a diagnostic of the electron beam properties and were not specifically optimizing the source yield. 


\subsubsection{Source control and comparison with conventional synchrotrons}

Although betatron X-ray radiation has tremendous potential for applications, some of which have begun, it still needs to be improved. The source needs to be better controlled and tuned, produce more photons and be more reproducible from shot to shot to be routinely used for applications. Its poor reproducibility is due to the highly nonlinear nature of the blowout regime of laser-wakefield acceleration and its sensitivity to nonideal laser beam and plasma density profiles [106]. In current blowout LWFA experiments, electron beam spectra typically have less than $10 \%$ energy spread and fluctuations between 1-10 MeV per shot. Several methods to control the stability and injection of electron beams have been proposed and some of them demonstrated experimentally. These include colliding-pulse injection [50, 29], density gradient injection [59, 61], inner-shell ionization-induced trapping [65, 66, 69], cold injection [55], and transverse control of self-injection using counter-propagating lasers [56] or external magnetic fields [57]. For the case of colliding-pulse injection, it yields more controllable and tunable betatron radiation [107]. Betatron radiation should be produced and observed in these experimental conditions to understand if the methods to control the injection and stability of the electron beams have an effect on the output reproducibility. Eventually, most of the betatron source parameters should be controlled with careful laser settings. The polarization can be controlled by tailoring the laser intensity profile and pulse front tilt $[108,91]$, and the spectrum by tailoring the laser wavefront with coma [109].

\subsection{Compton Scattering}

\subsubsection{Principle of Inverse Compton scattering with laser wakefield accelerators}

Inverse Compton scattering [110] is a process whereby a laser photon scatters off a relativistic electron to have its energy upshifted. In the literature several experiments are referred to as Thomson scattering, which is the classical limit where the wavelength is much greater than the Compton wavelength $\left(\lambda_{c}=h / m c=2.426 \times 10^{-12} \mathrm{~m}\right.$ for the electron, where $h$ is the Planck constant). While a number of experiments described in this review have been realized in the Compton and Thomson regimes (photon energies respectively above and below 511 keV), we will only use "Compton scattering" throughout the rest of the manuscript. In their rest frame, electrons see the Doppler upshifted electromagnetic field from the laser, and scatter the light in a classic dipole radiation pattern. A second upshift arises when returning to the lab frame. From energy-momentum conservation, one can derive the relativistic Doppler shifted energy of Compton scattered X-rays:

$$
E_{x}=\frac{2 \gamma^{2}(1-\cos \phi)}{1+\gamma^{2} \theta^{2}+a_{0}^{2} / 2+2 \gamma k_{0} \lambda_{c}} E_{p h}
$$

where $\gamma$ is the electron relativistic factor, $\phi$ is the angle between the incident laser and electron beams, $k_{0}=2 \pi / \lambda$ is the laser wavenumber, $a_{0}$ the laser normalized vector potential, and $E_{p h}$ is the laser photon energy. The electron recoil, $2 \gamma k_{0} \lambda_{c}$, can be neglected for modest LWFA energies and laser intensities. If the scattering laser vector potential is such that

$a_{0}<<1$, Compton scattering emission is mostly fundamental (in the case of a head-on collision, $\phi=180^{\circ}$, the scattered energy observed on-axis roughly scales as $4 \gamma^{2} E_{p h}$ ). At 
higher laser intensity $a_{0}>1$, the Lorentz force due to the magnetic field begins to become significant, the electron motion is more complex, and the radiation spectrum will contain higher harmonics of the laser frequency. Compton scattering sources are very attractive because one can obtain high-energy (beyond $100 \mathrm{keV}$ ) scattered photons with relatively modest electron beam energies, when compared to betatron X-ray radiation produced in the same regime $[89,111]$.

An important feature of Compton scattering sources is that the Compton scattering crosssection is very small $\left(\sigma=6.65 \times 10^{-25} \mathrm{~cm}^{-2}\right)$, so a high density of electrons and photons (and thus very high-quality beams) are required at the interaction point. In the case where the laser focal spot and electron bunch focus have similar size, $w_{0}$, the number of X-rays produced can be approximated by $N_{x}=\left(\sigma / \pi \omega_{0}^{2}\right) N_{L} N_{e}$, where $N_{L}$ and $N_{e}$ are respectively the number of laser photons and the number of electrons in the bunch. As the electron beam focal spot size scales as $1 / \gamma$, the X-ray yield varies as $\gamma^{2}$. Among LWFA-driven light sources, betatron radiation provides the highest brightness in the 10-100 keV range, but Compton scattering sources become a more efficient option at higher energies.

The advent of high intensity laser systems rapidly prompted many theoretical studies of Compton scattering in the nonlinear regime [112, 113, 114, 115, 116, 117], but not necessarily from LWFA electron beams. In the first theoretical Compton-LWFA source study, simulations (using 3D Compton scattering codes for the radiation and PIC codes for the electron beam phase space) predicted that with $300 \mathrm{MeV}, \mathrm{nC}$ electron beams with a $5 \%$ energy spread, $10^{7} \mathrm{X}$-ray photons per pulse could be produced, with energies up to $1 \mathrm{MeV}$ [118]. In this scheme, nonlinear spectral broadening of the scattered X-ray can be reduced by chirping the scattering laser pulse, which yields a significant increase in source brightness [119]. More recent simulations predict, for a $100 \mathrm{pC}, 200 \mathrm{MeV}$ electron beam, $10^{11}$ photons in a broad spectrum peaking at $10 \mathrm{MeV}$, with approximately $2 \%$ conversion efficiency of laser energy into gamma rays, in a beam collimated to less than 10-mrad divergence [120].

\subsubsection{Compton scattering experiments with laser wakefield accelerators}

Producing Compton scattering X-ray and gamma-ray sources from LWFA electrons is extremely challenging because it requires the synchronization of electron and laser beams to within a few femtoseconds and a few microns. Figure 5 and Table 2 summarize the properties of LWFA-driven Compton light sources at various laser facilities. Some of their notable features are also illustrated in Figure 6. The first experimental demonstration was done with a $10 \mathrm{TW}$ laser and a $180^{\circ}$ geometry, which produced Maxwellian electron distributions with temperatures around $6 \mathrm{MeV}$ and scattered photons with detectable energies up to $2 \mathrm{keV}$ [121]. The first Compton scattering experiment producing hard X-rays (up to $300 \mathrm{keV}$ ) was done with a single laser pulse and a foil placed at the exit of the LWFA to reflect the laser back onto the $\sim 100 \mathrm{MeV}$ electrons via a plasma mirror effect [111]. This scheme produced $10^{8}$ photons per pulse, and more recent studies have demonstrated the tunablility (75-200 $\mathrm{keV}$ ) of this method, as well as a quasi-monoenergetic (50\%) spectrum [122]. The main drawback remains the laser-to-X-ray photon conversion efficiency, on the order of $10^{-12}$.

Technological difficulties were recently overcome to produce gamma-ray photons between 1-4 MeV with two laser beams overlapping at $170^{\circ}$ [97]. Here, $\sim 300 \mathrm{MeV}$ electrons were produced by a $1.9 \mathrm{~J}, 35 \mathrm{fs}$ pulse focused onto a $2 \mathrm{~mm}$ gas target $\left(1 \times 10^{19} \mathrm{~cm}^{-3}\right.$, with $99 \%$ 
Table 2: Compton scattering $\mathrm{X}$-ray source and corresponding laser and plasma parameters obtained at a number of facilities worldwide. Quantities $E_{1}, \tau_{1}, w_{0,1}, a_{0,1}$ and $E_{2}, \tau_{2}, w_{0,2}, a_{0,2}$ are for the main laser and scattering laser pulse, respectively. For experiments using a foil, only values for the drive laser are given. Most of these sources can operate at a $10 \mathrm{~Hz}$ repetition rate at best.

\begin{tabular}{|l|l|l|l|l|l|l|l|l|}
\hline $\begin{array}{l}\text { Laser fa- } \\
\text { cility }\end{array}$ & $\begin{array}{l}\text { Pulse } \\
\text { energy } \\
E_{1}, \quad E_{2} \\
(\mathbf{J})\end{array}$ & $\begin{array}{l}\text { Pulse } \\
\text { dura- } \\
\text { tion } \\
\tau_{2}(\mathbf{f s})\end{array}$ & $\begin{array}{l}\text { Spot } \\
\text { size } \\
(\mu \mathbf{m})\end{array}$ & $a_{0,1}, a_{0,2}$ & $\begin{array}{l}\text { Electron } \\
\text { density } \\
\left(\mathbf{c m}^{-3}\right)\end{array}$ & $\begin{array}{l}\text { Photons } \\
\text { /shot }\end{array}$ & $\begin{array}{l}\text { X-ray } \\
\text { energy } \\
(\mathbf{k e V})\end{array}$ & Year \\
\hline $\begin{array}{l}\text { Salle } \\
\text { Jaune } \\
\text { (LOA) }\end{array}$ & 1 & 35 & 17 & 1.2 & $10^{19}$ & $10^{8}$ & $50-200$ & $\begin{array}{l}2012 \\
{[111]}\end{array}$ \\
\hline $\begin{array}{l}\text { DIOCLES } \\
\text { (U. Ne- } \\
\text { braska) }\end{array}$ & $1.9,0.5$ & 35,90 & 20,22 & $1.9,0.4$ & $10^{19}$ & $10^{7}$ & $70-9000$ & $\begin{array}{l}2013 \\
123,124]\end{array}$ \\
\hline $\begin{array}{l}\text { ATLAS } \\
\text { (MPQ) }\end{array}$ & $1.2,0.3$ & 28,28 & 13,25 & $4.4,0.9$ & $5 \times 10^{19}$ & $N . A$. & $5-42$ & $\begin{array}{l}2015 \\
{[125]}\end{array}$ \\
\hline $\begin{array}{l}\text { JETI } \\
\text { (Jena) }\end{array}$ & 0.333, & 85,85 & $\sim 3,3$ & $3,0.8$ & $6 \times 10^{19}$ & $3 \times 10^{4}$ & $0.4-2$ & $\begin{array}{l}2006 \\
{[121]}\end{array}$ \\
\hline $\begin{array}{l}\text { UT3 (UT } \\
\text { Austin) }\end{array}$ & 0.8 & 30 & 12 & 1.6 & $\begin{array}{l}1.4-2.2 \times \\
10^{19}\end{array}$ & $2 \times 10^{7}$ & $75-200$ & $\begin{array}{l}2015 \\
{[122]}\end{array}$ \\
\hline $\begin{array}{l}\text { Astra- } \\
\text { Gemini } \\
\text { (RAL) }\end{array}$ & 18,18 & 42,42 & 27, N.A. & $5.4,2$ & $3.2 \times 10^{18}$ & $10^{7}$ & $\begin{array}{l}6000- \\
18000\end{array}$ & $\begin{array}{l}2014 \\
{[126]}\end{array}$ \\
\hline
\end{tabular}

He and $1 \% \mathrm{~N}_{2}$ ), and a portion of the main beam was split to be focused onto the electrons at intensities around $10^{17} \mathrm{~W} / \mathrm{cm}^{2}$. The $4 \gamma^{2}$ scaling law of this source was subsequently demonstrated by the same group at X-ray energies between $100 \mathrm{keV}$ and $1 \mathrm{MeV}$ [123], as well as $9-15 \mathrm{MeV}$ gamma-rays $\left(3 \times 10^{5}\right.$ photons/shot) with a scattering laser doubled in frequency by a KDP crystal [124]. Tunable (5-42 keV), quasi-monochromatic and reproducible X-rays have also been reported from a more modest laser system (60 TW), with the presence of spectral nonlinear features [125]. At present, the highest photon energy obtained from a LWFA Compton source is $18 \mathrm{MeV}$ (approximately $10^{7}$ photons with energies exceeding 6 $\mathrm{MeV}$ per shot), form the $18 \mathrm{~J}, 40$ fs Astra-Gemini laser facility [126].

\subsubsection{Comparison with Compton scattering from RF accelerators}

The development of Compton scattering X-ray and gamma-ray sources with conventional accelerators is also a very active field of research. Characteristics of some of these current sources are described in Table 3. The first Compton machine producing gamma-rays was demonstrated at SLAC in the late 60s [127], and the first source specifically conceived for applications of Compton-scattered gamma-rays was built in Italy in the late 70s [128]. Several sources have been built by utilizing free-electron laser (FEL) radiation from an electron bunch and by interacting it with the next electron bunch in a storage ring, but conventional optical lasers offer much better beam quality. Similarly, sources rely on many different accelerator technologies: Linac, energy recovery linac, or storage rings. Currently, the narrower bandwidth of Compton sources from RF accelerators makes them more desirable for precision nuclear physics applications. A Compton source with photon energies up to $19.5 \mathrm{MeV}$ 


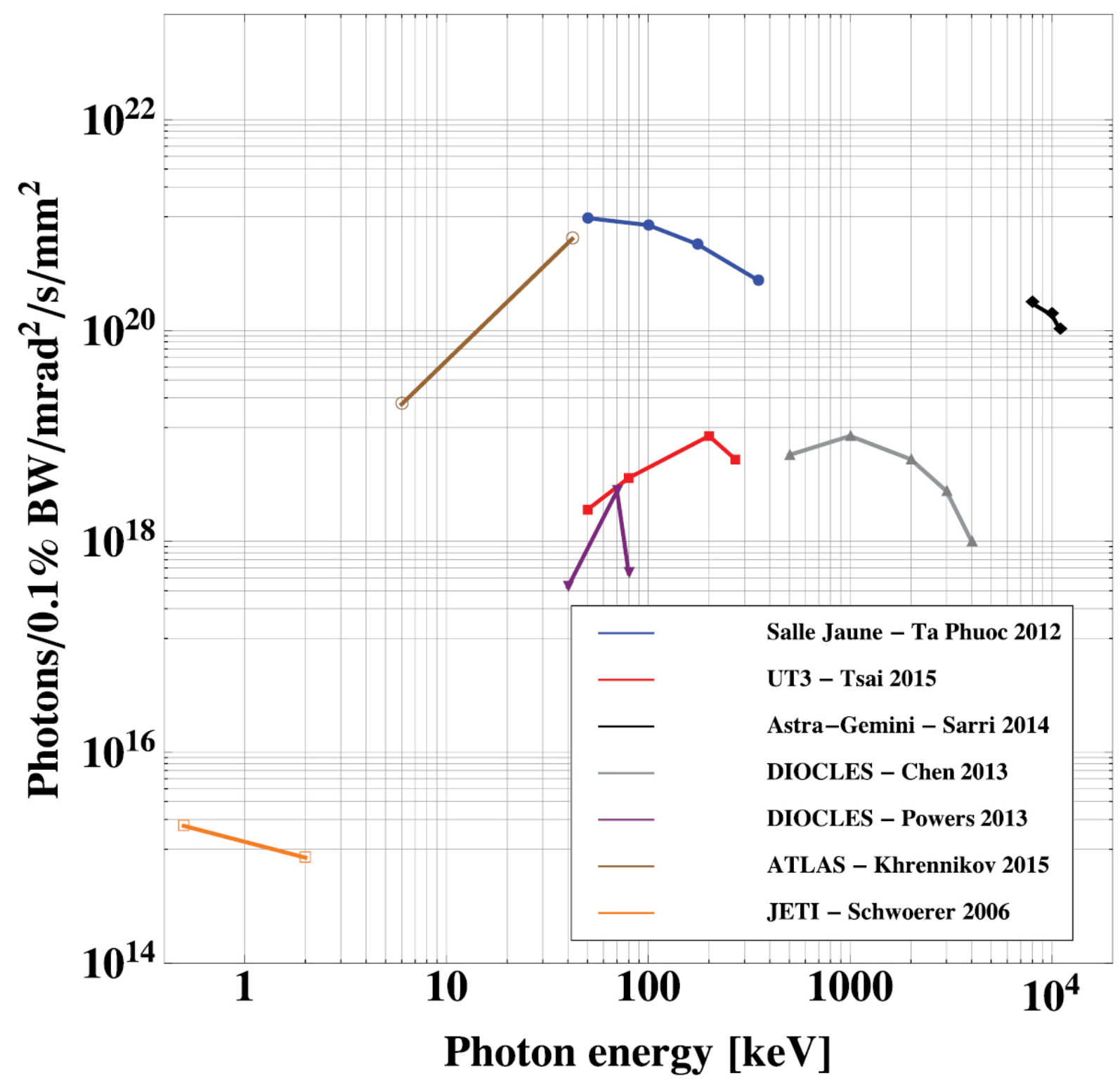

Figure 5: Peak brightness of Compton scattering X-ray sources produced at the facilities listed in Table 2. Here LWFA electrons oscillate in the electrical field of a laser pulse to emit high photon energy x-rays or gamma-rays. The experiments of Ta Phuoc [111] and Tsai [122] were performed using a foil acting as a plasma mirror to reflect the laser pulse onto the accelerated electrons. In the experiments of Chen [97], Powers [123], Khrennikov [125], Sarri [126] and Schwoerer [121] the electrons oscillate in a second laser pulse, which counter propagates with respect to the LWFA driver. 

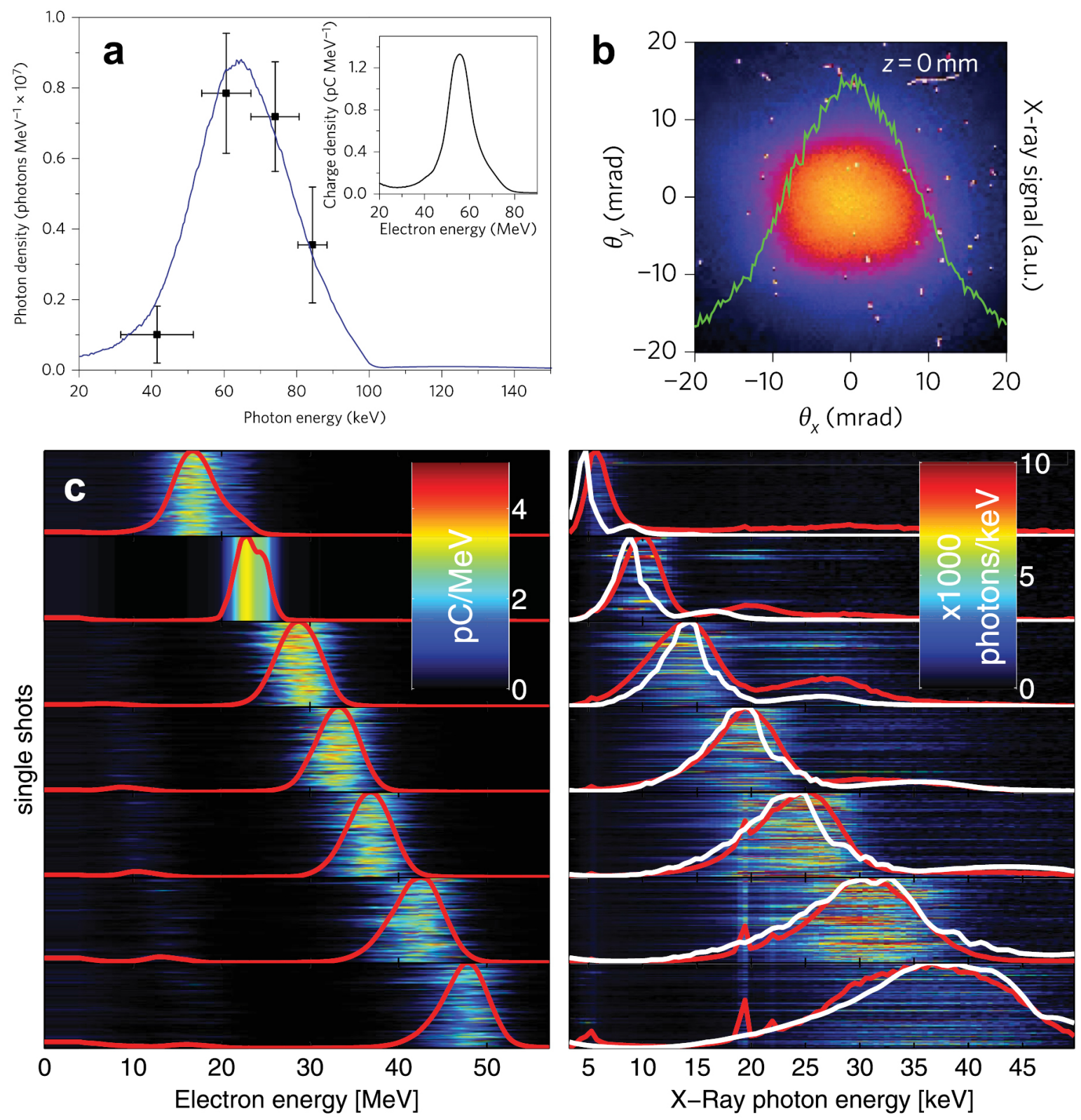

Figure 6: Notable features of Compton scattering light sources from LWFA. a: narrow band spectrum obtained from quasi-monoenergetic electron beams, from Powers et al [123]. b: collimated, hard (>100 keV) X-rays, from Ta Phuoc et al [111]. c: $4 \gamma^{2}$ on-axis scaling of the X-ray energy with the electron relativistic factor $\gamma$, from Khrennikov et al [125]. 
Table 3: Recent Compton scattering $\mathrm{X}$-ray and $\gamma$-ray sources with RF accelerator technology. FB = Fabry-Perot cavity. CW = Continuous Wave. OR = Optical Recirculator.

\begin{tabular}{|c|c|c|c|c|c|c|c|c|}
\hline Facility & Accelerator & $\begin{array}{l}\text { Electron } \\
\text { energy } \\
{[\mathrm{MeV}]}\end{array}$ & Laser & $\begin{array}{l}\text { Laser } \\
\text { energy } \\
{[\mathbf{J}]}\end{array}$ & $\begin{array}{l}\text { Photon } \\
\text { energy } \\
{[\mathrm{MeV}]}\end{array}$ & $\begin{array}{l}\text { Spectral } \\
\text { band- } \\
\text { width } \\
{[\%]}\end{array}$ & $\begin{array}{l}\text { Flux } \\
{[\text { pho- }} \\
\text { tons } / \mathrm{s}]\end{array}$ & Year \\
\hline $\mathrm{Hi} \gamma \mathrm{S}$ & Storage ring & 1200 & FEL & 0.15 & $1-158$ & $1-10 \%$ & $10^{8}$ & 1996- [131] \\
\hline $\begin{array}{l}\text { PLEIADES } \\
\text { (LLNL) }\end{array}$ & S-band Linac & $54-57$ & $\begin{array}{l}\mathrm{Ti} \quad: \\
\mathrm{Al}_{2} \mathrm{O}_{3}\end{array}$ & 0.4 & $0.04-0.08$ & $\sim$ few $\%$ & $10^{8}$ & $\begin{array}{l}\text { 2000-2004 } \\
{[132]}\end{array}$ \\
\hline $\begin{array}{l}\text { T-REX } \\
\text { (LLNL) }\end{array}$ & S-band Linac & 120 & $\begin{array}{l}N d: \\
Y A G\end{array}$ & 0.15 & $0.075-0.9$ & $\sim 10 \%$ & $10^{6}$ & $\begin{array}{l}2005-2008 \\
{[133]}\end{array}$ \\
\hline $\begin{array}{l}\text { NewSUBARU } \\
\text { (Japan) }\end{array}$ & Storage Ring & 1000 & $\begin{array}{l}\mathrm{Nd}: \\
\mathrm{YVO}_{4}\end{array}$ & $\mathrm{CW}$ & $6.6-17.6$ & $17 \%$ & $10^{5}$ & 2003- [134] \\
\hline THOM-X & Storage Ring & $50-70$ & $\begin{array}{l}\text { Fiber- } \\
\text { FB }\end{array}$ & $2.5 \times 10^{-6}$ & 0.05-0.09 & N.A. & N.A. & $\begin{array}{l}\text { In construction } \\
{[135]}\end{array}$ \\
\hline PHOENIX & $\begin{array}{l}\text { Superconducting } \\
\text { linac }\end{array}$ & 22.5 & $\begin{array}{l}\mathrm{Ti}: \\
\mathrm{Al}_{2} \mathrm{O}_{3}\end{array}$ & 0.1 & $\begin{array}{l}0.08- \\
0.013\end{array}$ & $4.5 \%$ & $10^{5}$ & 2013- [136] \\
\hline ELI-NP & S/C-band linac & 19.5 & $\begin{array}{l}\mathrm{Ti} \quad: \\
\mathrm{Al}_{2} \mathrm{O}_{3} \\
\text {-OR }\end{array}$ & 0.3 & $6.6-17.6$ & $0.5 \%$ & $\begin{array}{l}>5 \times \\
10^{8}\end{array}$ & $\begin{array}{l}\text { In construction } \\
{[129]}\end{array}$ \\
\hline
\end{tabular}

and a spectral bandwidth better than $0.5 \%$ is currently being constructed in Europe specifically for nuclear physics [129]. Although the precision of a LWFA-driven Compton scattering source is not yet at the level of RF accelerator sources, several projects in the US, which are showing very promising results, are specifically targeting nuclear forensics applications at LBNL [130] and the University of Nebraska [123].

\subsubsection{Radiation Reaction}

When a charged particle emits radiation, that radiation carries momentum and therefore this momentum must be accounted for. This is true whether the physical model being considered is a classical or quantum one. The loss of momentum by an emitting particle is known as radiation reaction, and as suggested by Equation 10, it can play a role in the photon energy of Compton scattering sources. We may assume that however difficult it may be to perform the calculation, radiation reaction is self-consistently contained within the framework of quantum electrodynamics. However, in classical dynamics, the force equation must be modified to include the effect of the radiation. The problem cannot be solved by simply taking the classical limit of the quantum equations in a straightforward way [137].

The radiation-reaction force was first formulated nonrelativistically by Lorentz, relativistically by Abraham and then self consistently by Dirac. The resulting equation has been a source of much controversy because it is a third-order differential equation that allows, for example, for self-accelerating solutions that do not conserve energy. Many authors have offered modified solutions [138]. These are generally identical to first order in the expansion proposed by Landau and Lifshitz [139],

$$
\frac{d}{d \tau} v^{\mu}=-\frac{e}{m_{e}} F_{\alpha}^{\nu} v_{\nu}\left[\eta^{\alpha \mu}-\tau_{0} \frac{e}{m_{e} c^{2}} v_{\beta} v^{\mu} F^{\alpha \beta}\right],
$$


where $v^{\mu}$ is the four-velocity, $\tau$ proper time, $F^{\alpha \beta}$ is the electromagnetic field tensor, $\tau_{0}=$ $e^{2} / 6 \pi \epsilon_{0} m_{e} c^{3}$ and $\eta^{\mu \nu}$ is the Minkowski metric tensor with trace -2. Quantum radiation reaction is essentially the overall recoil experienced by an electron undergoing multiple simultaneous incoherent photon emission events [140].

The importance of radiation reaction in general can be determined from the relative magnitude of the radiation force in Eq. 11 as

$$
\psi=\gamma^{2} a_{0} \frac{2 r_{e} \omega}{3 c},
$$

where $r_{e}$ is the classical electron radius, $\omega$ is the inverse time scale over which the particle is accelerated and $a_{0}=e A / m_{e} c$. Quantum radiation reaction is important when the parameter $\chi \gg 1$, where

$$
\chi=\frac{\left\|F_{\mu \nu} v^{\nu}\right\|}{c E_{c r}}
$$

[141], which corresponds to an electric field that is large compared with $m_{c}^{2} / e \lambda_{c}$.

Radiation reaction is intrinsically linked to light sources; radiation damping is well known in storage rings and synchrotrons. The inverse Compton energy loss in a single electron interaction with a single photon is similarly well known.

In laser-plasma interactions radiation reaction can be significant because $\omega$ is large, $a_{0}$ is large and $\gamma$ is large. The most significant manifestation of radiation reaction is in nonlinear inverse Compton scattering where the field strength in the rest frame of the electron is maximized and the acceleration timescale minimized. Under these conditions, where a relativistic electron collides head on with an intense $\left(a_{0}>1\right)$ laser field, the quantum parameter, $\chi$, can be written as [141]

$$
\chi=\frac{2 \hbar}{m_{e} c^{2}} \omega_{0} \gamma_{0} a_{0} .
$$

Therefore by controlling the values of $a_{0}$ and $\gamma$, different regimes can be explored. In the classical radiation reaction, calculations have shown that significant energy losses due to radiation reaction can be achieved with the highest intensity lasers colliding with electron beams accelerated to $\sim 100 \mathrm{MeV}$ energies $[142,120]$. For GeV energy beams, quantum radiation reaction $[143,140,144,145,146,147,148,149]$ starts to dominate, and generation of electron-positron pairs starts to ensue as $\chi$ approaches 1 through the multiphoton BreitWheeler effect $[141,150]$. This is where the energetic inverse Compton scattered photons absorb a large number of laser photons and decay into an electron positron pair. This process was first observed on the SLAC facility [151]. Experimental verifications of nonlinear QED predictions will be likely to be undertaken simultaneously with laser wakefield driven inverse Compton schemes. In addition, understanding of quantum effects may allow new sources for applications, such as the demonstration of a quasi-monochromatic very bright source of photons from an ultrarelativistic electron beam colliding with a thermonuclear plasma due to kinematic pileup [152].

\subsection{Undulator and XFEL radiation}

Since X-ray free electron lasers (XFEL) became available to users [3], they have revolutionized many scientific fields by allowing the observation of ultrafast and ultra small phenomena. 
However such facilities require kilometer-long accelerators to operate. LWFAs now produce electron energies [37] comparable to what is needed for an XFEL, and are being considered as a desirable approach to produce XFELs with a compact footprint. A lot of progress has been made to produce LWFA beams with small emittances and large peak current, however the large electron energy spread (a few \%) severely hinders the prospect of a LWFA-based XFEL. This can be understood by looking a the basic principles of a XFEL [153]. When an electron passes through a periodic undulator with a magnetic field $B_{0}$ and period equal to $\lambda_{0}$, then it emits radiation in the forward direction at the wavelength:

$$
\lambda=\frac{\lambda_{0}}{2 \gamma^{2}}\left(1+\frac{K_{0}^{2}}{2}\right),
$$

where $K_{0}=0.934 B_{0}[$ Tesla $] \lambda_{0}[\mathrm{~cm}]$ is the undulator strength, and $\gamma$ the electron relativistic factor. As the undulator strength increases beyond unity, harmonics of the fundamental radiation are emitted, as illustrated in Figure 7. A plane electromagnetic wave with wavelength $\lambda$ can thus exchange energy with the electron beam. Depending on their phase with respect to the wave, electrons either gain or lose energy, leading the electron beam to be microbunched with $\lambda$ periodicity and to consequently emit coherent radiation. When the wave gains energy, XFEL amplification occurs until saturation is reached. From Eq. 15, a spread in electron beam energy means a spread in $\lambda$, which prevents microbunching and thus XFEL gain. In a high gain FEL, the Pierce parameter [153]

$$
\rho=\frac{1}{4 \gamma}\left(\frac{I}{I_{A}} \frac{K_{0}^{2}[J J]^{2} \lambda_{0}^{2}}{\pi^{2} \sigma_{x}^{2}}\right)^{1 / 3}
$$

limits the acceptable electron beam energy spread $\sigma_{\gamma}$ to $\sigma_{\gamma} / \gamma<<\rho$. Here, $I$ is the electron beam peak current, $I_{A} \sim 17 \mathrm{kA}$ the Alfvén current, $\sigma_{x}$ the rms beam size and $[J J]=$ $J_{0}(Y)-J_{1}(Y)$ the field coupling. $J_{0}$ and $J_{1}$ are Bessel functions and $Y=K^{2} /\left(4+2 K^{2}\right)$.

Because current LWFA electron beam properties do not satisfy the requirements for an XFEL, several undulator schemes have been numerically investigated to overcome the large energy spread of LWFA beams. A detailed discussion on this subject is presented in Reference [5]. A transverse field variation into the FEL undulator reduces the effect of beam energy spread and jitter, and this method shows that it can produce soft X-rays from $1 \mathrm{GeV}$ electron beams with a $1 \%$ rms energy spread, for a $5 \mathrm{~m}$ long undulator with a periodicity of $1 \mathrm{~cm}$ [155]. In this scheme, the electron beam energy and the undulator parameter are given a transverse linear dependence so that every electron satisfies the resonance condition (Equation 15). Another concept has shown that FEL gain can be obtained for EUV rays with an electron beam energy spread of $1 \% \mathrm{rms}$ and a bunch charge of $5 \mathrm{pC}$ by using a large undulator strength $>1$ and bunch decompression [156]. The electron energy considered was $300 \mathrm{MeV}$ and the FEL wavelength was calculated to $\lambda=134 \mathrm{~nm}$. A chicane can also be used as a bunch stretcher [157], which reduces the gain length for an XFEL operating with an energy spread on the order of the Pierce parameter. Space-charge effects due to the high peak beam currents (10-100 kA) of LWFAs are a problem as well. Solutions include the generation of electron beams with a negative energy chirp (the energy in the front of the beam is lower than at the tail) or the use of a tapered (variable period) undulator [158]. Thomson scattering was proposed as a method for conditioning FEL electron beams [159]. 

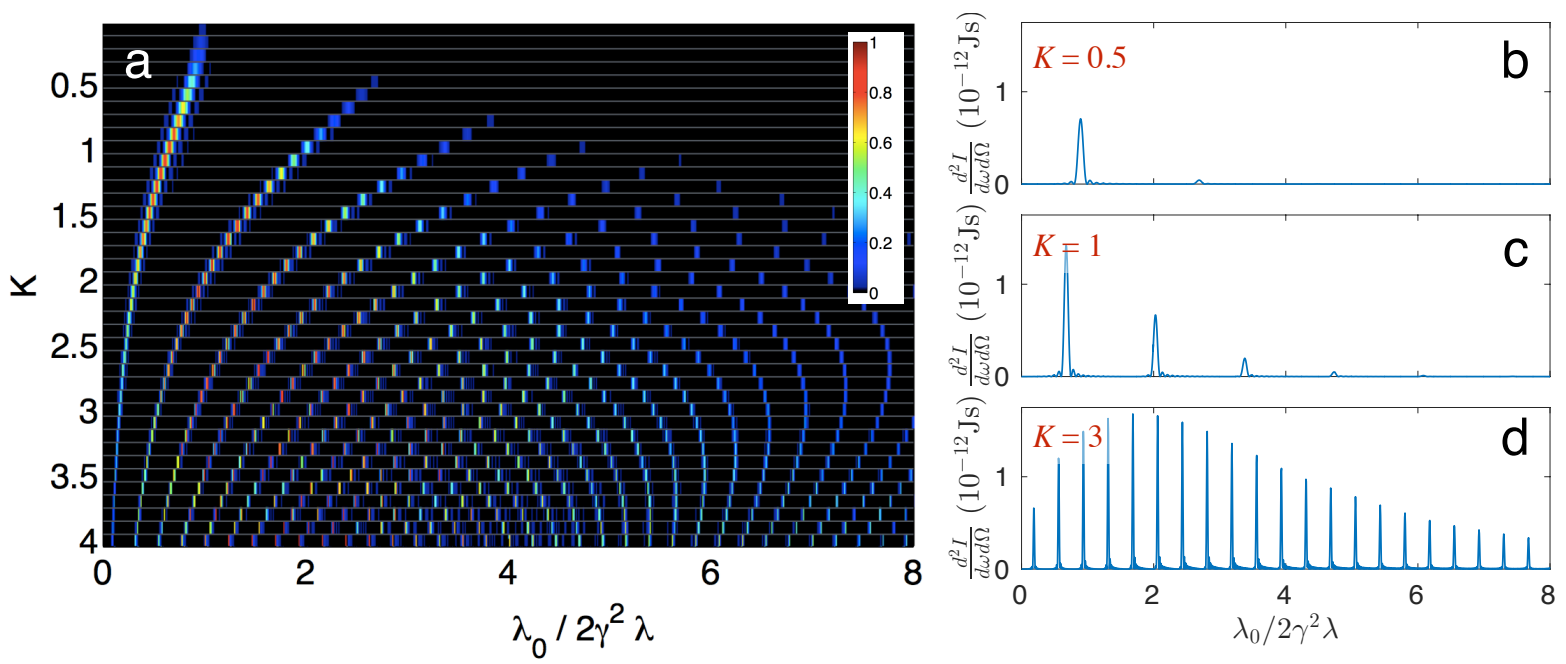

Figure 7: Illustration of undulator radiation as a function of the wiggler strength. For $K<<1$, a relativistic electron oscillating through the wiggler emits at the fundamental wavelength $\left(\lambda=\lambda_{0} / 2 \gamma^{2}\right)$. As $K$ increases beyond unity, higher order harmonics are emitted, with the fundamental redshifting to longer wavelengths, until they ultimately merge into a continuous broadband spectrum. (a) On axis photon energy spectrum $d^{2} I / d \omega d \Omega$ as a function of inverse undulator wavelength relative to $2 \gamma^{2} / \lambda_{0}$ and wiggler parameter $K$ for an electron traversing a 3 period magnetic undulator with Lorentz factor $\gamma$. (b-d) The same data for 3 different values of $K$. Calculated using RDTX [154].

In this case, Thomson scattering of an intense focused laser pulse produces a correlation between the energy loss by an electron and its transverse location in the laser field.

Unfortunately, experiments have not yet demonstrated an XFEL driven by LWFA electron beams. So far, only undulator radiation has been produced, with $55-75 \mathrm{MeV}, 1 \%$ energy spread electron beams in the visible wavelengths [160], and with $200 \mathrm{MeV}$ electron beams in the soft X-ray range, down to a few nm [161]. Although there are still many experimental and technological challenges to overcome, several institutions worldwide are actively pursuing the design of LFWA XFELs at the SOLEIL synchrotron [162], at LBNL [163] and at SACLA.

\subsection{Bremsstrahlung}

\subsubsection{Theory}

As hot electrons propagate through a target, collisions with the background particles, in particular ions, generate high energy radiation emission by bremsstrahlung. Bremsstrahlung generally means radiation emission by a deflection in particle momentum over a short period of time, with a characteristic flat spectrum for a single deflection for frequencies short compared with the inverse collision time and exponentially falling off for higher frequencies.

When considering Coulomb collisions, the differential radiation cross section is [77]:

$$
\frac{d^{2} \chi}{d \omega d Q}=\frac{d I(\omega, Q)}{d \omega} \frac{d \sigma_{S}}{d Q}
$$


where $d I(\omega, Q) / d \omega$ is the spectral energy emitted per unit frequency interval for a collision with momentum transfer $Q=\sqrt{\Delta p^{2}}$ and $\sigma_{S}$ is the Rutherford scattering cross section.

When integrated over all possible momentum transfers (equivalent to different impact parameters) this can be expressed as

$$
\frac{d \chi}{d \omega}=\frac{16}{3} \frac{Z^{2} e^{2}}{c}\left(\frac{e^{2}}{m_{e} c^{2}}\right)^{2} \frac{1}{\beta^{2}} \ln \left(\frac{Q_{\max }}{Q_{\min }}\right),
$$

where $Q_{\max }$ and $Q_{\min }$ are the maximum and minimum momentum transfers in a collision. For an electron traveling with velocity $\beta c$ in a field of ions with density $n_{i}$, the spectrum of photons emitted per unit time is therefore:

$$
\frac{d P}{d \omega}=\frac{16}{3} Z^{2} n_{i} e^{2}\left(\frac{e^{2}}{m_{e} c^{2}}\right)^{2} \frac{1}{\beta} \ln \left(\frac{Q_{\max }}{Q_{\min }}\right)
$$

Bethe and Heitler [164] first calculated an expression for the radiation cross section for non relativistic quantum emission, and an ultra relativistic $E \gg \hbar \omega$ cross section can also be found under the Born approximation.

The angular distribution of the bremsstrahlung is a dipole field in the plane of the collision for a non relativistic collision, and for a relativistic collision is peaked in the direction of motion. An angular distribution that is consistent with both the relativistic and non relativistic cases is

$$
\frac{d^{2} P}{d E d \Omega}=\frac{1}{\hbar} \frac{d P}{d \omega}\left[\frac{3}{16 \pi} \frac{1}{\gamma^{2}(1-\beta \cos \theta)^{2}}\left(2+\frac{\cos ^{2} \theta-1}{\gamma^{2}(1-\beta \cos \theta)^{2}}\right)\right] .
$$

This is the radiated power in bremsstrahlung photons per unit photon energy per unit solid angle for a single electron propagating through a homogenous plasma of ion density $n_{i}$. Screening can also be an important effect in some materials; atomic electrons shield the nuclei and modify the collision.

\subsubsection{Bremsstrahlung produced by LWFA electrons}

LWFA electrons have been employed to produce gamma-rays by passing through high-Z material converters. This technique has distinct advantages over direct laser irradiation of solid targets. One of the first experiments reporting on the generation of bremsstrahlung gamma rays from a LWFA was conducted at the Rutherford Appleton Laboratory in the self-modulated regime [165]. Picosecond laser pulses were focused to intensities $\sim 5 \times 10^{19}$ $\mathrm{W} / \mathrm{cm}^{2}$ either directly onto a Tantalum target or onto a gas jet to accelerate electrons that subsequently collided with the solid target. The main observation was that in the LWFAdriven case, the source is more collimated $\left(5^{\circ}\right)$ than in the direct drive case $\left(>45^{\circ}\right)$. Similar experiments were later reproduced with a femtosecond laser system [166]. Electron beams (20-200 MeV), with Maxwellian temperatures around $40 \mathrm{MeV}$, interacting with a Tantalum converter, were used to produce gamma-rays with a small source size $(320 \mu \mathrm{m})$ and low divergence (a few degrees). The source duration is expected to be on the order of the laser pulse duration (30 fs), and the photon dose is on the order of 1 Gy a few centimeters from 
the source. As a comparison, tumors are typically treated with photon doses on the order of $10^{\prime} s$ of Gy.

The source size can be further reduced to $30 \mu \mathrm{m}$ with proper optimization of the electron beam parameters [167]. Approximately $10^{8}$ photons were generated in the 8-17 MeV gammaray region by 10-45 MeV LWFA electron beams produced with a $10 \mathrm{TW}$ laser system and crossing a $2 \mathrm{~mm}$ Tantalum slab [168]. The yield of gamma-rays produced by LWFA electrons from a similar laser system exceeds the yield of gamma-rays from direct irradiation of solid targets by two orders of magnitude [169]. Progress made on LWFA electron beams now permits the production of tunable gamma-ray sources based on bremsstrahlung. A $\sim 15 \mathrm{pC}$, $220 \mathrm{MeV}$, quasi-monoenergetic electron beam can produce $10^{9}$ photons around $10 \mathrm{MeV}$ [170]. A $300 \mathrm{pC}, 1 \mathrm{GeV}$, electron beam traversing a $1 \mathrm{~mm}$ Tungsten target would produce $6 \times 10^{11}$ photons/shot, with $0.1 \%$ of these having an energy greater than $15 \mathrm{MeV}$.

\section{6 $\mathrm{THz}$ and coherent transition radiation}

Transition radiation occurs when electrons traverse a medium with a discontinuity in dielectric properties. It means that the electrical fields are different in the two media: these must reorganize and this discrepancy is released as transition radiation. The radiation is coherent if the electron bunch is shorter than the emission wavelength, in which case the intensity of the radiation scales quadratically (as opposed to linearly for incoherent radiation) with the electron bunch charge. In a LWFA, coherent transition radiation (CTR) is generated by the electron beam induced polarization currents at the boundary of the plasma/vacuum interface (typically in the $\mathrm{THz}$ regime, with submillimeter wavelengths), before the bunch can spread and lengthen [171]. In the limit of a semi-infinite plasma, with dielectric constant $\epsilon=1-\omega_{p}^{2} / \omega^{2}$, the energy of CTR scales as $W \propto N^{2}\left(u \times \theta_{0}\right)^{4}$, where $N$ is the electron bunch charge, $u$ the electron energy, and $\theta_{0}$ the opening angle collecting the radiation.

CTR in the terahertz region from a LWFA was observed for the first time in 2003 in the self-modulated regime, with electron spectra of temperatures equal to 4.6 MeV [172]. Here coherent radiation in the $0.3-3 \mathrm{THz}$ range was generated, with $0.3 \mu \mathrm{J}$ per pulse within a 100 mrad angle. Since then, CTR has been mainly used as a diagnostic for LWFA electrons. Of particular interest we note the electron bunch duration and longitudinal profile, which have been measured with various techniques. Examples include phase retrieval of CTR spectra [173], single-shot $\mathrm{THz}$ time-domain interferometry [174], electro-optic sampling, where the $\mathrm{THz}$ field induces a change in birefringence in an electro-optic (EO) crystal $[175,176,177]$ and single-shot 2D spatial THz imaging [178].

Measurements of submicron structures in the electron beam can only be made if the radiation is in the visible domain. In LWFAs, optical CTR can be obtained by placing a radiator (typically a thin foil with solid density) in the path of the electron beam. Optical CTR is produced at the boundary of the foil. Very fine structures, attributed to microbunching of the beam, have been measured with optical CTR in the 400-1000 nm range [179], and bunches as short as 1.8 fs $r m s$ with a few kilo amperes of peak power have been reported by using controlled optical injection [29, 180]. Optical CTR has also shown that the energy spread at a given slice (in the longitudinal coordinate of the bunch) can be significantly smaller (below the percent level) than the energy spread of the full electron beam [181]. 


\section{Medical and biological applications}

One potential application for the high energy photons generated by laser wakefield accelerators is in medicine and biology. Radiation use in medicine in general is divided into two main areas; diagnostic radiology and radiotherapy. Diagnostic radiology is the use of radiation to enable determination of physiology and body function and for the diagnosis of disease. Radiotherapy is the use of high energy radiation for treating cancer. Both of these areas may use energetic photon sources to achieve these goals, for example X-rays for imaging or directly applied for radiation therapy, but also the use of $\gamma$-rays for production of radioisotopes.

The most common radiation source for diagnostic radiology is X-rays, which are used extensively in the medical fields. The first use of X-ray radiation for imaging was Wilhelm Roentgen's absorption image of his wife's hand in 1895. Following this discovery, X-ray imaging was adopted for medical use incredibly rapidly, with real-time X-ray imaging using scintillating screens being available within years of the discovery. The invention of computed tomography (CT) scanning techniques in the 1970s has given doctors an extraordinary ability to look noninvasively into a patients body and to study form and function in detail. CT works by constructing a 3D map from a large number of 2D images. CT machines are used extensively, with 150 scans per 1,000 people on average according to the Organisation for Economic Co-operation and Development, but at the cost of significant dose to patients $[182]$.

In X-ray absorption radiography, the standard technique in diagnostic radiology, dense structures such as bone can be easily distinguished from soft tissues through the difference in $\mathrm{X}$-ray absorption. Sometimes a contrast agent, such as a barium meal for the gastrointestinal system, may be used to increase attenuation of X-rays since the absorption of X-rays in soft tissues can be marginal for lower energy X-rays. The attenuation of the transmitted photon number can be expressed as $N=N_{0} \exp [-(\mu / \rho) \rho x]$, where $\mu / \rho$ is the mass attenuation coefficient for material of density $\rho$. As shown in Table 4, lower energy X-rays are effectively attenuated by the body, which contributes to absorbed dose in the body with no imaging benefits. At around the K- $\alpha$ energy of Tungsten, approximately $60 \mathrm{keV}$, there is generally good contrast. However, clearly for all energies absorption differences between e.g. soft tissue and adipose tissue are very small. X-ray tubes for absorption radiography can be produced relatively cheaply (of order $\$ 1000-\$ 100,000$ ) and efficiently by using an electron beam accelerated across a $10-300 \mathrm{kV}$ potential colliding with a high-Z metal anode, such as Tungsten. When the electron impacts it produces a characteristic spectrum that is a combination of bremsstrahlung and $\mathrm{K}-\alpha / \mathrm{K}-\beta$ components. High resolution can be achieved by using a sub-mm scale anode in an X-ray tube, a technology known as $\mu \mathrm{CT}$. The one limitation in this technology is that to generate a small source size for high resolution imaging, the X-ray flux is limited by melting of the anode if the current is too high [94].

Recent developments in X-ray imaging include detector improvements in resolution and sensitivity, energy resolution and monochromatic imaging and fast scanning techniques [184]. An important recent development has been that of X-ray phase contrast imaging [185], which has become a new tool for potential diagnosis in medicine. This involves imaging the interference pattern occurring between X-rays that have accumulated different phase when passing through phase gradients in material and is much more sensitive than absorption 
Table 4: Table of mass attenuation coefficients and relative attenuation through $2 \mathrm{~cm}$ of medically relevant materials for $10 \mathrm{keV}, 60 \mathrm{keV}$ and $200 \mathrm{keV}$ X-rays (data from Ref. [183]).

\begin{tabular}{|c|c|c|c|c|c|c|}
\hline Material & $\begin{array}{c}\mu / \rho\left(\mathrm{cm}^{2} \mathrm{~g}^{-1}\right) \\
@ 10 \mathrm{keV}\end{array}$ & $\begin{array}{c}\mu / \rho\left(\mathrm{cm}^{2} \mathrm{~g}^{-1}\right) \\
@ 60 \mathrm{keV}\end{array}$ & $\begin{array}{c}\mu / \rho\left(\mathrm{cm}^{2} \mathrm{~g}^{-1}\right) \\
@ 200 \mathrm{keV}\end{array}$ & $\begin{array}{c}N / N_{0}(2 \mathrm{~cm}) \\
@ 10 \mathrm{keV}(\%)\end{array}$ & $\begin{array}{c}N / N_{0}(2 \mathrm{~cm}) \\
\text { @ } 60 \mathrm{keV}(\%)\end{array}$ & $\begin{array}{c}N / N_{0}(2 \mathrm{~cm}) \\
@ 200 \mathrm{keV}(\%)\end{array}$ \\
\hline Adipose Tissue & 3.3 & 0.20 & 0.14 & 0.2 & 69 & 78 \\
Soft Tissue & 5.4 & 0.20 & 0.14 & $10^{-3}$ & 65 & 74 \\
Bone & 29 & 0.31 & 0.13 & 0 & 34 & 63 \\
Lead & 130 & 5.0 & 1.0 & 0 & 0 & $10^{-8}$ \\
\hline
\end{tabular}

imaging. For small phase gradients, the angle $\Delta \theta$ that an $\mathrm{X}$-ray propagating in the $z$ direction is deflected by due to a phase gradient in the $x$ direction is [185]

$$
\Delta \theta \approx-\frac{r_{e} \lambda^{2}}{2 \pi} \int \frac{\partial n_{e}}{\partial x} d z
$$

where $\lambda$ is the X-ray wavelength and $r_{e}$ is the classical electron radius. Interfaces between tissues are highlighted in particular, since these correspond to stronger phase gradients. This can be very beneficial in imaging of tumors situated within tissue with similar absorption properties. Phase contrast imaging has the additional benefit that harder X-rays may be used such that with the resulting lower absorption, less dose is delivered to a patient.

Phase contrast imaging can also be performed with broadband X-rays [186, 187, 188, 189, 190], since only the spatial coherence of the X-rays is important for forming the first peak of the interference pattern that highlights the edge. One way of achieving spatial coherence without complex X-ray optics is by allowing a point X-ray source to diffract sufficiently at a distance away. This is known as propagation based imaging.

\subsection{Diagnostic radiology using LWFA X-ray sources}

LWFA X-ray sources have some potential for medical applications. Since LWFA betatron Xrays naturally emanate from a small source inside the plasma wakefield (with sources reported as small as $100 \mathrm{~nm}[80]$ ), are bright, high energy and have a degree of tunability, the LWFA source is suitable for single shot, lensless phase contrast imaging. Typical peak energies generated by current LWFA experiments, Table 1, are already close to the ranges needed for various radiographic procedures, as highlighted in Table 5. LWFA sources generate X-rays with a critical energy that is to some degree tunable and there is also potential to develop more monochromatic and significantly higher energy X-ray sources. Moreover, properties such as the short pulse duration could potentially lead to new concepts for advanced imaging in the future. However, it remains to be seen if the cost of such a system would be competitive with mature medical X-ray technologies.

Phase contrast imaging with the LWFA betatron source has typically been propagation based imaging. This technique has been used in LWFA experiments for imaging biological samples. Some examples of phase contrast images of biological objects acquired with betatron $\mathrm{X}$-ray radiation are shown in Fig. 8. Fig. 8A shows a typical set-up for X-ray phase contrast imaging experiments. $\mathrm{B}$ and $\mathrm{C}$ show phase contrast images of insects $[191,192]$ and D 
Table 5: Typical ranges of energies used for various procedures. Adapted from Ref. [184].

\begin{tabular}{|c|c|}
\hline Type & Energy range (keV) \\
\hline Mammography & $17-25$ \\
Angiography & $33 \mathrm{keV}$ (Iodine K edge) \\
Radiography & $40-60$ \\
CT & $60-70$ \\
\hline
\end{tabular}

shows absorption images of bone samples [94]. Studies have demonstrated soft tissue phase contrast imaging [193] and extraction of quantitive information by phase retrieval has also been performed [98]. An inverse Compton source using a conventional accelerator has been used for biological imaging [194] and such monoenergetic and potentially high energy X-ray sources are of great potential benefit [195] since they can be tuned to maximize contrast at an absorption edge, for example. LWFA enabled inverse Compton imaging is expected to be demonstrated in the near future.

\subsection{Nuclear medicine}

Nuclear medicine is a branch of radiology in which radioisotopes are used for either treatment (such as by implantation of a radioactive isotope inside cancerous tissue to destroy the cancer) or diagnosis, as in positron emission tomography (PET), for example. PET is a technique where a $\beta_{+}$radioisotope is attached to a molecule, such as glucose. On annihilation of the emitted positron, two antiparallel gamma rays are generated that are detected and used to pinpoint where the radioisotope is concentrated, in a tumor for example. One of the most widely used procedures using a radioisotope is single photon emission computed tomography (SPECT), which is when a $\gamma$-ray emitting source, an isomeric state, is used in a similar way to PET, but with lower spatial resolution. The main advantage of SPECT is its low cost due to the availability and long life of the radioisotopes. A particularly important isomer is the technetium isomer Tc-99m that is used in over $80 \%$ of procedures. The shortlived technetium isomer is "milked" from a Molybdenum-99 source. The Mo-99 is generally acquired by extraction from the spent fuel of a nuclear reactor. Finding alternative sources to nuclear reactors is desirable, as there have been worries about shortages of these vital radioisotopes [196].

Laser plasma interactions may be a source of important radionuclides in the future [197]. One way of generating isotopes is through photo-nuclear reactions, i.e. a nuclear reaction that causes transmutation or fission to new radioisotopes triggered by an energetic gamma ray. For example, 6-7\%, depending on the photon energy [198], of U-235 photofissions will generate the Mo-99 isotope needed for SPECT through the reaction

$$
\gamma+{ }^{235} \mathrm{U} \rightarrow{ }^{99} \mathrm{Mo}+{ }^{136-X} \mathrm{Sn}+X n
$$

An inverse Compton source could be used to produce a tunable, monochromatic source for photofission medical isotope production [199, 200]. The use of laser wakefield accelerators to generate high energy gamma rays through bremsstrahlung has been demonstrated and used 


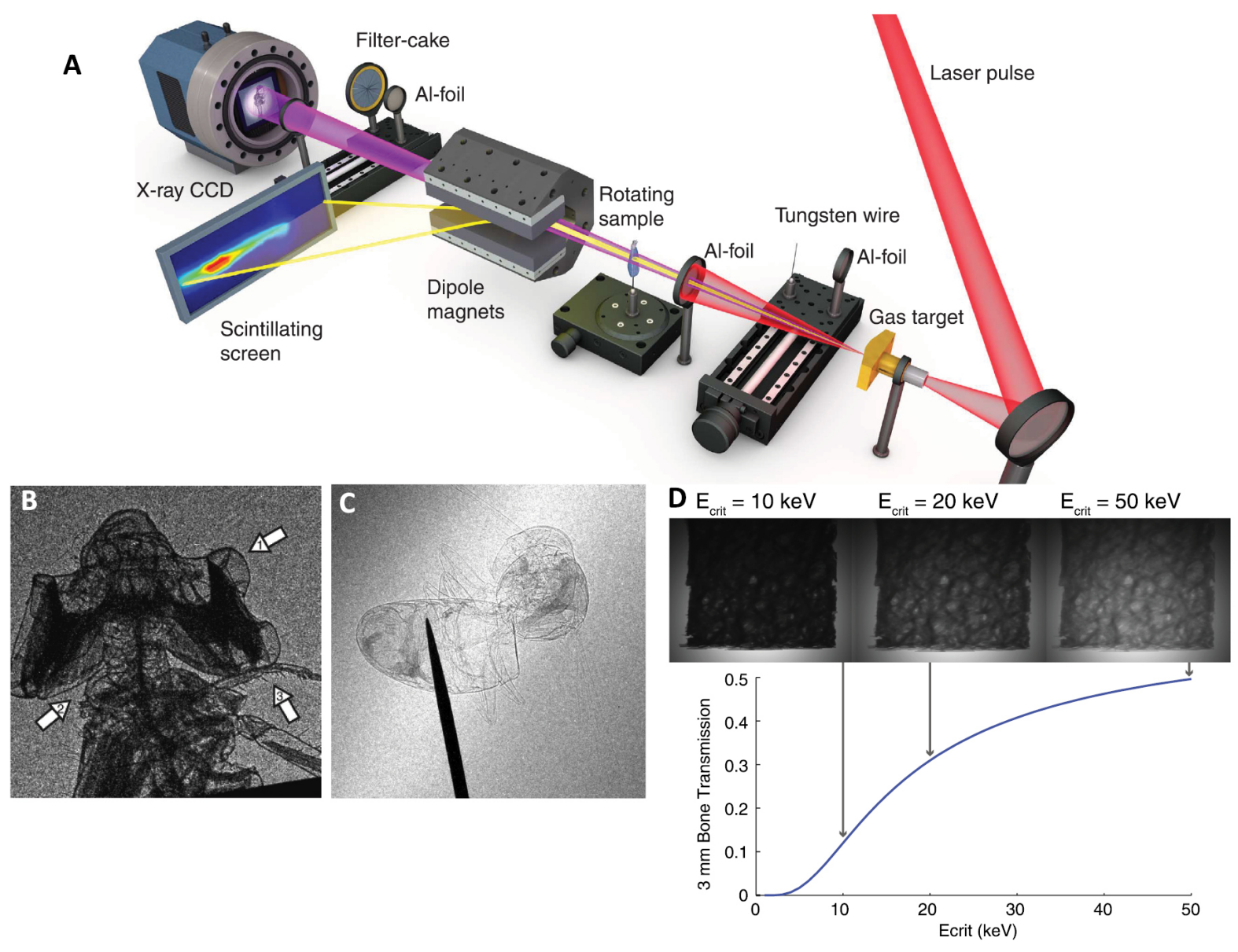

Figure 8: X-ray phase contrast imaging with betatron radiation. (A) Typical experimental setup, from Wenz et al. [98]. (B) Single shot phase contrast image of the head of a damselfly recorded using a betatron source with a critical energy $E_{c}=10 \mathrm{keV}$. From Kneip et al. [192] (C) Single shot phase contrast image of a bee recorded using a betatron source with a critical energy $E_{c}=12 \mathrm{keV}$ and source size of $1.7 \mu \mathrm{m}$. From Fourmaux et al. [191]. (D) Human trabecular hip bone sample imaged with a betatron source at three different critical energies, and corresponding transmission curve through $3 \mathrm{~mm}$ of bone sample, from Cole et al [94]. 
for photofission of natural Uranium [201] and for activation by $(n, \gamma)$ reactions [201, 202, 203]. In these studies, laser wakefield accelerated electrons of energies from $25 \mathrm{MeV}$ up to 500 $\mathrm{MeV}$ interacted with a high-Z target to generate bremsstrahlung photons with energies in a broad spectrum up to the electron energy, as in section 2.5. The yields were measured using high purity Ge detectors and scintillators. Photofission yields of up $10^{5}$ per shot were demonstrated [201].

\subsection{Biomedical applications of $\mathrm{THz}$ radiation}

$\mathrm{THz}$ radiation can also be used for biomedical applications, by making use of the low frequency modes of biologically relevant molecules. In particular, water has a strong absorption peak for $\mathrm{THz}$ radiation and therefore $\mathrm{THz}$ can be used for identifying tumors in epithelial tissue by differences in water content, for example [204]. THz radiation is non ionizing and can penetrate deeper than optical radiation because of reduced scattering. Imaging and computed tomographic imaging [205] with $\mathrm{THz}$ radiation are other biomedically relevant applications. Preliminary imaging measurements have been performed using the $\mathrm{THz}$ emitted when a LWFA electron bunch emerges from the plasma-vacuum interface [178].

\section{Military, defense and industrial applications}

\subsection{High resolution gamma-ray radiography}

Gamma-ray radiography is widely used for non destructive evaluation [206] in a number of applications such as the inspection of cargo containers or welded structures (pipes, vessels, tanks) produced by large industries. Compton scattering and bremsstrahlung from LWFAs present several advantages that are desired for this application: high energy photons (MeV) to penetrate dense objects, a low dose for safety, and a small source size (a few $\mu \mathrm{m}$ ) for good spatial resolution.

Short pulse laser/solid interactions can directly produce gamma-rays suitable for radiography. High intensity laser pulses $\left(>10^{18} \mathrm{~W} / \mathrm{cm}^{2}\right)$ are absorbed into hot electrons with a temperature on the order of the ponderomotive potential [207]. These hot electrons, observed in early experiments [208, 209], can produce copious amounts of gamma-rays with solid, high-Z targets such as Gold or Tungsten [210, 211]. At intensities of $10^{19} \mathrm{~W} / \mathrm{cm}^{2}, 10^{4}$ photons/eV/Sr have been reported, and were used to radiograph a high area density object (up to $85 \mathrm{~g} / \mathrm{cm}^{3}$ ) [211]. The drawback is the rather large source size, about $400 \mu \mathrm{m}$, which limits the spatial resolution.

A better resolution can be achieved with bremsstrahlung produced by the interaction of LWFA electrons (in the self-modulated [165] and blowout [166] regime) with a solid target. The resolution in the first blowout regime experiment was $320 \mu \mathrm{m}$ [166], which was well improved to $30 \mu \mathrm{m}$ with optimization of the electron beam parameters [167, 212]. The results of some of these gamma-ray imaging experiments are presented in Figure 9.

Compton scattered X-rays and gamma-rays are also considered as a promising source for this application. Gamma ray photons with energies up to $10 \mathrm{MeV}$ and a spectral bandwidth of $10 \%$ have been measured for potential radiography applications [213]. In this experiment, 

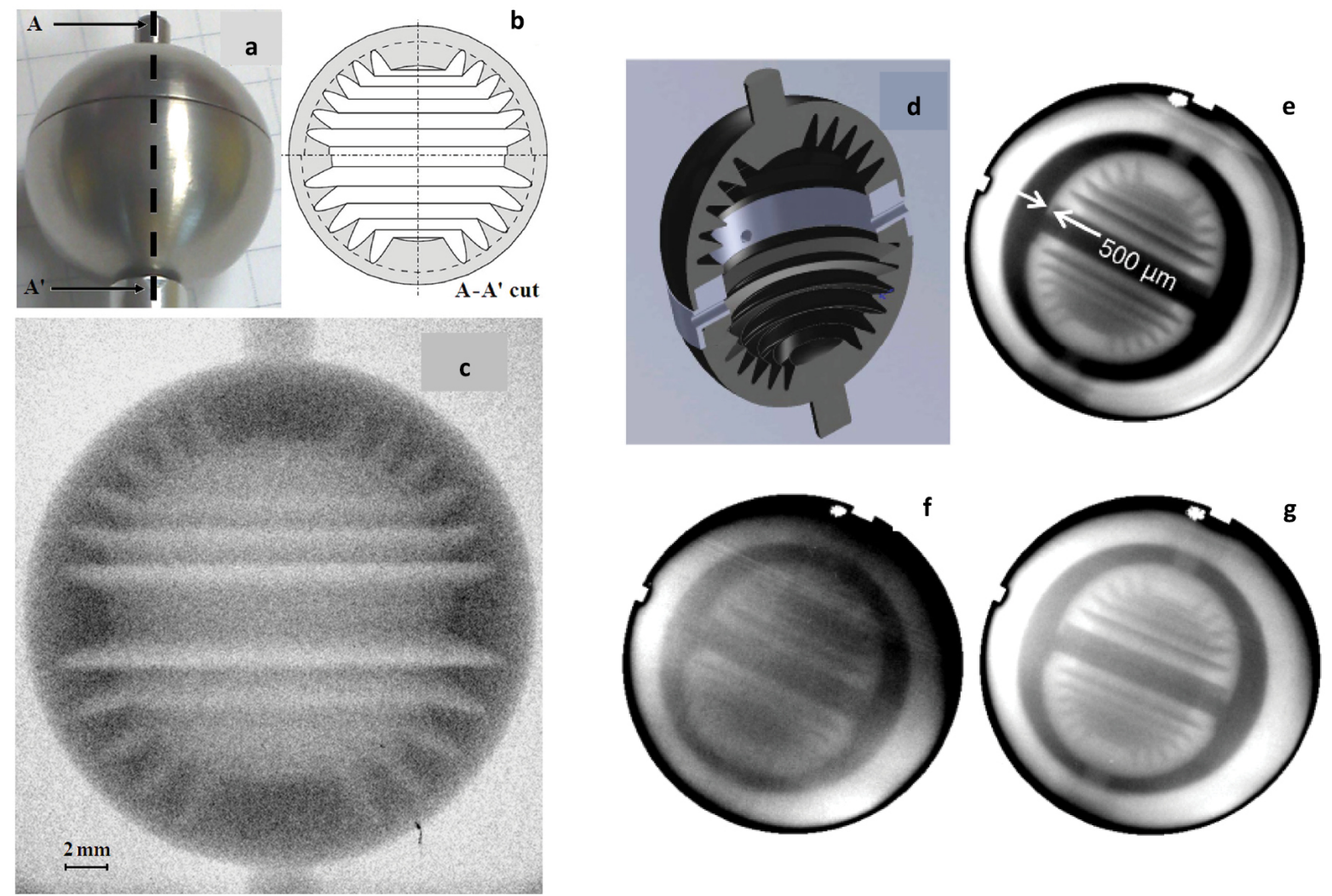

Figure 9: Gamma ray radiographs of a test object recorded with two different sources. (a) Photo of a $20 \mathrm{~mm}$ diameter Tungsten object, (b) a schematic A-A $\mathbf{A}^{\prime}$ cut, and (c) the resulting radiograph with a LWFA driven bremsstrahlung gamma-ray source. From Ben-Ismail et al. [167] (d) CAD section view of another similar, $20 \mathrm{~mm}$ diameter test object, and radiography images, obtained with bremsstrahlung from direct laser-solid interaction through (e) $66 \mathrm{~g} / \mathrm{cm}^{2}$, (f) $102 \mathrm{~g} / \mathrm{cm}^{2}$, and (g) $85 \mathrm{~g} / \mathrm{cm}^{2}$ areal densities. From Courtois et al. [211]

using a driver for the electron beam and a scattering beam, the X-ray source size was measured to be $5 \mu \mathrm{m}$ using a cross-correlation technique in which the scattering pulse is scanned. In addition, the narrow divergence of the beam is well suited for long standoff imaging. A USB flash drive was radiographed with a $1.5 \mu \mathrm{m}, 100 \mathrm{keV}$ Compton scattering X-ray source [111], with observation of very small features.

\subsection{Backscattered X-ray and gamma-ray inspection}

Backscattered X-rays and gamma-rays are a tool for the long-range identification of concealed objects. They are also useful for the detection of explosive devices and land mines [214]. For homeland security inspection, a few requirements are necessary, and have to allow detection from long distances (more than several meters), which is not possible with standard X-ray tubes since the backscattered signal is very weak. LWFA-driven sources deliver a low dose of bright X-rays and gamma-rays within a sub-picosecond pulse that can be backscattered by an object of interest. The relation between the scattered X-ray energy $E_{x}^{\prime}$ and the incident 
X-ray energy $E_{x}$ is given by:

$$
E_{x}^{\prime}=\frac{E_{x}}{1+\frac{E_{x}}{E_{0}}(1-\operatorname{Cos} \theta)},
$$

where $E_{0}=0.511 \mathrm{MeV}$ is the electron rest energy and $\theta$ the angle between the incident and scattered beam. The energy of the scattered radiation depends on the angle of observation, and the amount depends on the electron density and thickness of the material under investigation. This technique is typically more sensitive to X-ray energies of a few $100 \mathrm{keV}$, and it is commonly used to measure spectra from MeV-class sources [215]. Another method directly employs the LWFA electron beams to generate backscattered x-rays by the sample itself [?].

Several experiments have attempted to use laser-produced X-ray sources for this application, with relatively good success. A 1.2 TW Ti:Sapphire laser was focused onto a $0.5 \mathrm{~mm}$ Al foil to produce bremsstrahlung X-rays that were used to detect acrylic, Copper and lead blocks inside an Aluminum container [216]. In this experiment, the energy of the backscattered X-rays was within 70-200 keV and the object was placed about 1 meter from the source. The authors estimated that a $3 \mathrm{mrad}$ beam containing $10^{6}$ photons/pulse in this energy range would be sufficient to detect an object at a distance of 10 meters with this technique.

\subsection{Isotope-specific detection with nuclear resonance fluorescence}

Being able to detect specific isotopes with a minimal dose of radiation is of importance for homeland security, stockpile stewardship, or nuclear waste assay. Isotopes, which have the same number of electrons and protons, but differ by their number of neutrons, have exactly the same chemical signatures. For example, Uranium has two well known isotopes $\left({ }^{238} \mathrm{U}\right.$ and ${ }^{235} \mathrm{U}$ ) with very different usage and reactivity in a number of applications. Isotopes have distinct nuclear energy levels that can be used to specifically detect them, and that induce nuclear resonance fluorescence (NRF) [217], a process illustrated in Figure $10 \mathrm{~d}$. Here, an incident gamma-ray photon (typically $>0.5 \mathrm{MeV}$ ), is absorbed by the nucleus of a specific isotope. When this isotope relaxes back to equilibrium, it isotropically reemits photons at a slightly lower energy (due to recoil). For example, the absorption energy of ${ }^{7} \mathrm{Li}$ is $0.477629 \mathrm{MeV}$ and the corresponding $\mathrm{NRF}$ line emission energy is $0.477595 \mathrm{MeV}$. In principle, NRF lines are characterized by a very strong cross section and a narrow linewidth. Several approaches to calculate the NRF cross sections exist in the literature, and include different broadening mechanisms. For example, Metzger [218] includes thermal broadening and the effective NRF absorption near the resonant energy $E_{R}$ is:

$$
\sigma(E)=\sigma_{0} \frac{\pi^{1 / 2}}{2} \frac{\Gamma}{\Delta} \exp \left[-\left(\frac{E-E_{R}}{\Delta}\right)^{2}\right]
$$

Here, $\Gamma$ is the total width of the excited state and

$$
\sigma_{0}=\left(\frac{\lambda_{r}^{2}}{2 \pi}\right)\left(\frac{2 J_{1}+1}{2 J_{0}+1}\right)
$$


where $J_{1}$ and $J_{0}$ are the total angular momenta of the excited state and the ground state, respectively, and $\lambda_{r}$ the corresponding wavelength of the resonant energy. The actual NRF absorption lines are much wider than their natural linewidths because of Doppler-broadening. If for a nucleus at rest the NRF radiation has the energy $E$, then the energy $E^{\prime}$ for a nucleus moving towards the source with a certain velocity $v$ is going to be shifted according to $E^{\prime} \sim E(1+v / c)$, where $c$ is the speed of light. If the velocities of the nuclei are distributed according to a Maxwellian distribution function, then the Doppler width of the NRF line is:

$$
\Delta=E_{R}\left(\frac{2 k T_{e f f}}{M c^{2}}\right)^{1 / 2},
$$

where $M$ the mass of the nucleus, $k$ the Boltzmann constant, and $T_{\text {eff }}$ the effective temperature of the material. This model is valid as long as $\Gamma+\Delta>>2 k T_{D}$ where $T_{D}$ is the Debye temperature. Because in most cases $\Gamma<<1 \mathrm{eV}$, the total width is just determined by the thermal motion of the atoms and for the majority of gamma-ray transitions $\Delta / \Gamma>100$. Despite Doppler-broadening, the NRF lines exhibit a relative energy width of $\Delta E / E \sim 10^{-6}$, which is well below the energy resolution of standard germanium-based detectors. Therefore it is desirable to use a gamma-ray source with a narrow energy linewidth to efficiently excite and detect NRF lines. One could argue that the best solution is to excite the NRF line of a given isotope with the same isotope as the source of exciting radiation. However, recoil energy losses upon emission and absorption prevent us from doing this. Indeed, the recoil yields a shift toward lower energies and the line emitted is off resonance by:

$$
\Delta E_{R}=E^{2} / M c^{2}
$$

In the case of ${ }^{7} \mathrm{Li}$, which has an $\mathrm{NRF}$ line at $0.478 \mathrm{MeV}$ and a nucleus of atomic weight 7 , $\Delta E_{R} \sim 35 \mathrm{eV}$. It is larger than the natural linewidth and than the Doppler width at room temperature $(\sim 1.33 \mathrm{eV})$.

Based on these theoretical considerations, narrow bandwidth gamma-ray sources offer the best alternative to efficiently excite and detect NRF transitions. NRF detection has been demonstrated with broadband bremsstrahlung sources [219, 220], but the best prospect for this application appears to be Compton scattering sources, which can be tunable and spectrally narrow if well designed. Several Compton X-ray and gamma-ray sources with rf accelerator technology have been used to perform NRF detection in Japan [221], Duke University [222], and Lawrence Livermore National Laboratory [133, 215]. In principle, the electron beam parameters (emittance, energy spread) are easier to control in a rf accelerator. New projects are investigating LWFA-driven Compton scattering sources as a driver for, among other applications, NRF detection. Recent work that begun at the Lawrence Berkeley National Laboratory shows that with appropriate phasing of the injection of the electron beam and plasma lensing and guiding, bandwidths on the order of $1 \%$ are realistic [130, 223].

\subsection{Nuclear waste treatment and photo transmutation}

Gamma-rays from LWFA electrons (bremsstrahlung or Compton scattering) can induce nu-

clear reactions through transmutation, which can be applied to nuclear waste disposal and 

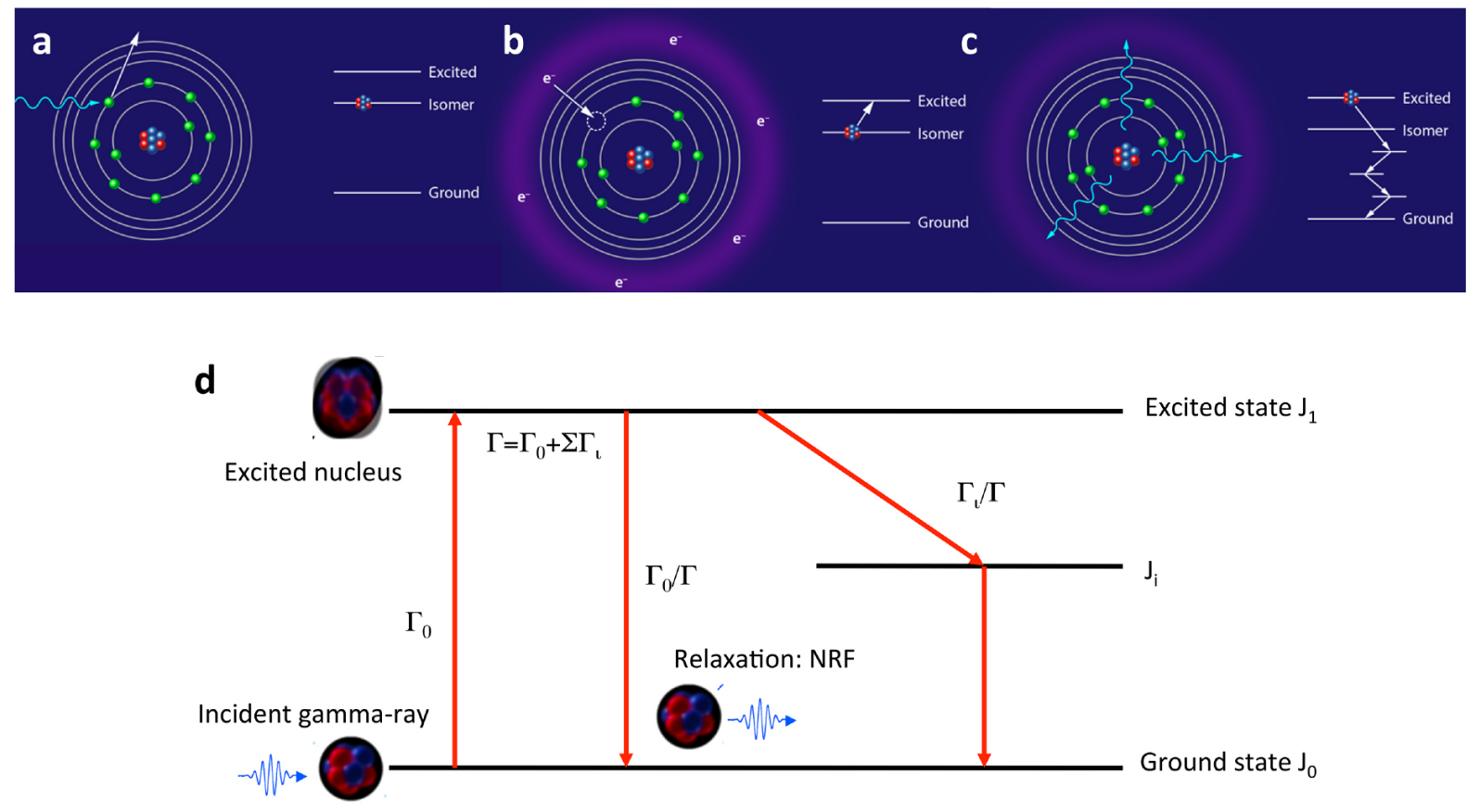

e

Incident gamma-ray

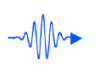

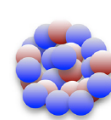
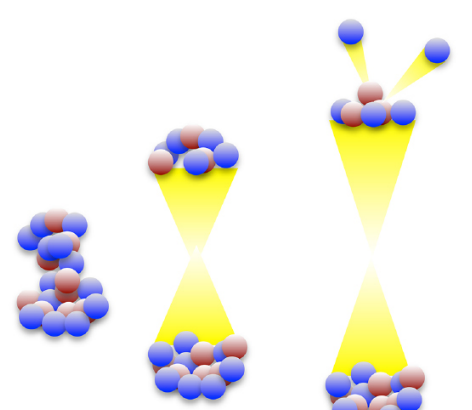

Figure 10: Illustration of nuclear processes that could be triggered by LWFA-driven X-ray and gamma ray sources. (a-c): NEEC. In (a), X-ray photons knock electrons out of inner atomic shells, and the nucleus remains in the metastable isomeric state. (b) electrons from the plasma are captured in the inner-shell vacancies, their excess energy excites the nucleus to a higher, less stable, energy state. (c) the nucleus relaxes to its ground state, releasing photons in a cascade. (d): NRF. An incident gamma-ray excites a nucleus from the ground state (angular momentum $J_{0}$ ) to the excited state (angular momentum $J_{1}$ ). $\Gamma_{0}$ is the partial width for the direct gamma-ray transition to the ground state. The nucleus relaxes back to the ground state by emitting a photon of characteristic energy. (e) photofission. An incident gamma-ray is absorbed by a high-Z nucleus, which breaks into two smaller nuclei and releases neutrons. 
active interrogation. Transmutation is the changing of a nucleus. Artificially, this is triggered by particles (electrons, protons, neutrons), which we will not discuss here, or by gamma rays.

The byproducts of nuclear power plants are long-lived radioactive isotopes. Examples include ${ }^{129} \mathrm{I}\left(T_{1 / 2}=1.57 \times 10^{7}\right.$ years $),{ }^{99} \mathrm{Tc}\left(T_{1 / 2}=2.1 \times 10^{5}\right.$ years $)$ and ${ }^{126} \mathrm{Sn}\left(T_{1 / 2}=2.3 \times 10^{5}\right.$ years). Long-term storage and disposal of these isotopes is a real problem, since they need to be buried deep underground. One solution is to transmute them into smaller, short-lived isotopes with gamma-rays from high intensity lasers [224].

Photofission reactions produce prompt and delayed fission gamma-rays and neutrons and can be used as an active interrogation tool [225]. An illustrative example is shown in Figure 10 e. The photon yield needs to be large enough to produce signals, but below the photoneutron threshold to reduce dose and background. It is not trivial, because photofission cross sections are smaller than neutron cross sections, and neutrons are better shielded with low Z material while gamma-rays are better shielded with high Z material. Photofission of ${ }^{238} \mathrm{U}$ has actually been demonstrated with direct laser-driven bremsstrahlung sources, for laser intensities on target of about $10^{20} \mathrm{~W} / \mathrm{cm}^{2}$ [226], as well as with LWFA-driven bremsstrahlung [201]. However, bremsstrahlung sources, whether they are produced by electrons from a conventional accelerator or from a LWFA, are impractical for this application because of their large spectral width. For photofission of ${ }^{235} \mathrm{U}$, approximately $10^{9}$ photons are needed within a $10 \%$ bandwidth, and the photon energy has to be larger than $\sim 5$ $\mathrm{MeV}$ [223]. Currently, the best performance of a LWFA-driven Compton scattering source is $10^{6}-10^{7}$ photons within a $10 \%$ bandwidth at $13 \mathrm{MeV}$ [213], which makes this application within reach.

\subsection{Energy storage: nuclear excitation by electron capture}

As opposed to isotopes having a different number of neutrons, a nuclear isomer refers to different states of a nucleus, but with the same number of neutrons and protons [227, 228]. Typical half-lives of nuclear excited states are on the order of 1 picosecond, whereas some isomeric states last several hours. They can be forced to relax back to equilibrium with different excitation processes that involve X-rays. For example, the interaction of X-rays with one of the isomeric states of Molybdenum, ${ }^{93 m} \mathrm{Mo}$, forces the nucleus to relax back to its ground state via two different mechanisms. ${ }^{93 m} \mathrm{Mo}$, initially at a $2.5 \mathrm{MeV}$ excitation energy, can be further excited with $4.85 \mathrm{keV}$ radiation before decaying back to a ground state. This direct photoexcitation process is rather inefficient and requires the X-rays to be resonantly tuned (within a few eV) to the nuclear resonance. However there is a concurrent, more efficient process enabling the same energy release from the isomer. In this case, called nuclear excitation by electron capture, or NEEC (Figure $10 \mathrm{a}-\mathrm{c}$ ), the interaction of the Xrays with the target produces plasmas with temperatures approaching a few $100 \mathrm{eV}$ if the intensity is sufficient [229]. A free electron from the plasma is captured into one of the inner-shells of the Mo atom, which in turn excites the nucleus from its initial isomeric state. This mechanism is obviously less sensitive to the resonance condition, and is six orders of magnitude more efficient than direct photoexcitation [230].

At present, only an XFEL like LCLS produces X-ray intensities sufficient to enable NEEC. Nuclear isomers are of interest for a number of applications in nuclear medicine [199], where they can be used for therapeutic (endo-radiotherapy) or diagnostic (nuclear imaging) pur- 
poses. For example, with ${ }^{93 m} \mathrm{Mo}$, a $1 \mathrm{MeV}$ photon is emitted in the decay cascade from the triggering level to the ground state, and it can potentially be detected by modern imaging systems or target a specific tumor. As for energy storage, the excess isomer energy that can be released through the interaction with the XFEL opens the way toward a clean, controlled nuclear energy source. Given the kilometer-size and availability of XFELs worldwide, it seems unlikely that this will be a practical energy-storage device, unless compact LWFA-driven XFELs can be demonstrated.

\subsection{Detection of explosives and drugs with $\mathrm{THz}$ spectroscopy}

Other dangerous materials, such as explosives and drugs of similar density and composition, are not easily identified with X-rays and gamma-rays. The prospect of coherent, LWFAdriven $\mathrm{THz}$ sources opens numerous possibilities in this domain [231]. Several materials have characteristic spectral features around a few $\mathrm{THz}$, which are attributed to intra- or intermolecular vibrational modes. As a result, $\mathrm{THz}$ spectroscopy can provide chemical and structural information. In addition, $\mathrm{THz}$ radiation is easily transmitted through paper, cardboard and plastic, which makes this technique very useful to detect drugs or explosives in a mail package. Many drugs have been investigated with $\mathrm{THz}$ time-domain spectroscopy. Examples include cocaine, ecstasy, amphetamine, and opiates, which all have clear spectral distinction over $0.2-2.5 \mathrm{THz}$ frequencies, which is typically the range of LWFA-driven $\mathrm{THz}$ sources [172].

Because of the low photon energy of $\mathrm{THz}$ radiation in comparison with X-rays, full body millimeter and $\mathrm{THz}$ wave scanners are now used at airports to detect metallic objects concealed behind paper or clothing. The typical frequency of these devices is around 100 $\mathrm{GHz}[232]$. Since the radiation is transmitted by antennas that rotate around the body, the small source size of LWFA-driven $\mathrm{THz}$ sources would probably be impractical for this application.

\subsection{Properties of semiconductors investigated with $\mathrm{THz}$ radiation}

Although not directly used in industry or security applications, some noteworthy properties of semiconductors are investigated with $\mathrm{THz}$ radiation. The few examples we describe in this section could potentially be investigated with LWFA-driven $\mathrm{THz}$ sources. In general, $\mathrm{THz}$ spectroscopy is a remarkable tool for probing low-energy excitations in semiconductors with ultrafast temporal resolution [233]. In semiconductors, the dynamics of free carriers is characterized by several parameters: the conductivity (frequency dependent), the plasma frequency $\omega_{p}$, and the carrier damping rate $\Gamma$ (the inverse of the carrier collision time). $\Gamma$ and $\omega_{p}$ typically have $\mathrm{THz}$ frequencies. Ultrafast $\mathrm{THz}$ Time-domain spectroscopy has been used to measure the complex conductivity of doped Silicon from low frequencies to frequencies higher than the plasma frequency and the carrier damping rate [234].

The absorption spectrum of a bulk semiconductor subjected to a strong electrical field develops an exponential tail below the band gap and oscillations above it, which is known as the Franz-Keldysh effect $[235,236]$. It can modify the band structure of the semiconductor at equilibrium. This effect has been observed by applying a strong THz field to a GaAs sample and by probing it with femtosecond white light [236]. In this experiment, the single-cycle 
THz pulses, with energies of $2 \mu \mathrm{J}$ and field strengths exceeding $100 \mathrm{kV} / \mathrm{cm}$, were generated by optical rectification in LiNbO3, and the probe beam was obtained by focusing a femtosecond laser pulse onto a thick sapphire plate. The THz pulse energies reported here are consistent with those measured in LWFA-driven THz sources [172]. In another experiment, THz pulses were used to observe stimulated $\mathrm{THz}$ emission from internal transitions of excitons in a $\mathrm{Cu} 2 \mathrm{O}$ semiconductor [237]. Broadband $\mathrm{THz}$ pulses, also produced by optical rectification, monitored the electromagnetic response of the sample after photo excitation.

\section{Condensed matter and high energy density science}

Transient phenomena are found in many states of matter and can only be investigated with pump-probe techniques, where radiation excites a sample of interest to a particular state, while another beam probes its evolution thereafter. In this section, we address some physics related to three distinct states: condensed matter (low temperatures), plasma (high temperatures), and warm dense matter (medium temperatures). These states of matter are illustrated in Figure 11, where we have indicated the conditions of relevant physical phenomena and experiments.

The field of high energy density (HED) science [6] is relatively young. One of its grand challenges is to understand the relationships between temperature, pressure, and density in extreme environments. For example, it studies fundamental questions regarding the evolution of the Sun, the formation of Jupiter, how to harness the power of stars to provide a limitless source of energy, or the mechanisms of stellar explosions. Scientists are now able to recreate in the laboratory HED conditions that were thought to be attainable only in the interiors of stars and planets. Although we know that lasers can be used to drive matter to extreme states of temperature and pressure, it is usually difficult to probe these laser-driven HED plasmas because most of the time they are in a non-equilibrium state and transient in nature.

Techniques that use external sources of particles and photons to probe these environments are advantageous because scientists can directly measure the effects that their interaction with an HED plasma creates. Unlike visible light, X-ray photons can pass through dense plasmas, and absorption of the X-rays can be directly measured, via spectroscopy or imaging. In this context, X-rays are one of the most versatile tools physicists can use to probe HED plasmas. Because of their remarkable spectral, spatial and temporal properties, light sources from LWFAs could have a tremendous potential for applications in HED science. Here we review some solid state and HED physics problems where LWFA light sources could have a big impact, and then discuss whether it would be an advantage to use them for specific experimental techniques.

\subsection{Condensed matter physics}

In condensed matter physics, scientists are interested by how atoms rearrange during phase transitions between different states (amorphous, liquid or crystalline). For most processes governed by atomic motion, the timescale of interest corresponds to one vibrational period, about $100 \mathrm{fs,}$ which is why LWFA-driven light sources can be potentially attractive for probing such a state of matter. For a very comprehensive review on this topic, which 


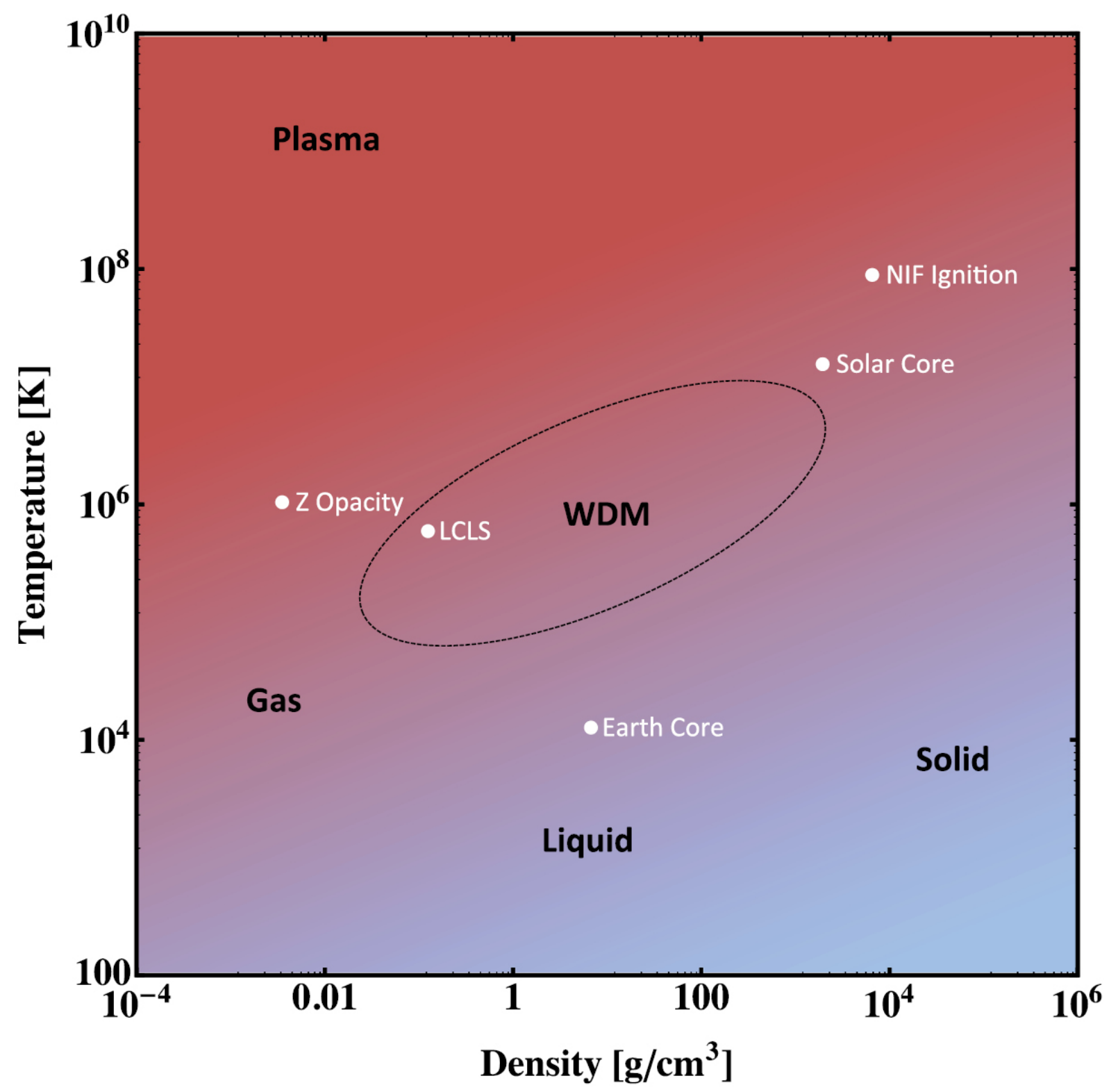

Figure 11: Temperature/density parameter space in HED science with conditions of interest for LWFA-driven X-ray and gamma-ray probes. We have indicated recent plasma conditions created at LCLS (Vinko et al [229]), the Sandia Z machine (Bailey et al [238]) and NIF (Lindl et al [239]) that could be probed with betatron X-rays or Compton scattered gamma-rays. 
goes beyond the scope of this paper, and on the type of sources that are commonly used for applications, we refer the reader to another review paper [240]. Structural changes in materials have been widely investigated with femtosecond crystallography [241, 242, 243] and usually require bright, monochromatic X-ray sources such as XFELs or line emission. More recent examples include the amorphous to crystalline phase transition in carbon [244] driven by XFEL pulses, the lattice dynamics of materials in which superconductivity is transiently induced by $\mathrm{THz}$ radiation [245], changes in long-range order during ultrafast melting in a perovskite manganite [246], or the spin dynamics in iron complexes [247].

\subsection{Laser-driven shocks}

Laser driven shocks are a reliable tool to compress materials, and their uniformity, steadiness and preheating can be well controlled [248, 249]. Pressures up to five terapascals, corresponding to conditions found at the interior of Jupiter, can be attained with ramp compression on lasers like the National Ignition Facility (NIF) [250]. Shocks are important in fusion energy research. For example, in the indirect-drive approach to inertial confinement fusion (ICF), the implosion is accomplished by compressing the fuel and capsule by a series of shocks [239]. The velocity of shocks in HED matter is currently measured with VISAR [251], however it is a surface diagnostic that does not provide detailed information about the shock front structure. High-energy X-ray backlighting and radiography have both undergone significant development in the past decade, and are now used to observe shocks in laser-driven HED experiments $[252,253,254]$. The spatial resolution of these diagnostics continues to be refined, and they can now address small-scale structure in HED plasmas. However, the temporal resolution of these measurements still needs to be improved. Third-generation synchrotron light sources have recently been used to provide single-shot, X-ray phase-contrast imaging of dynamic shocks; however, they are limited to temporal resolutions of 100 ps [255]. Femtosecond X-ray phase contrast imaging of shocks is now done at the Linac Coherent Light Source (LCLS) [256], but this capability cannot be replicated at other HED science facilities such as the National Ignition Facility (NIF) or OMEGA. Both betatron radiation and Compton scattering from LWFAs could provide a remarkable alternative to this problem.

\subsection{Electron-ion thermalization in warm dense matter}

When matter is driven off equilibrium by intense radiation, energy is first coupled to the electrons, and then the ions. This thermalization process between electrons and ions varies depending on the driver, but it happens within a few picoseconds or less. There are three types of driver that can produce warm dense matter states: nanosecond (ns) optical lasers, femtosecond (fs) optical lasers, and X-ray free electron lasers.

High energy, ns laser pulses drive matter to both high pressure and high temperature states, and subsequent electron-ion thermalization is observed. To obtain such conditions, long ns pulses are used, with large focal spot diameters (typically more than $100 \mu \mathrm{m}$ to avoid edge effects), and intensities around $10^{14} \mathrm{~W} / \mathrm{cm}^{2}$. The typical target is composed of an ablator of plastic (a few microns of $\mathrm{CH}$ ) followed by the material under study and a tamped layer (also $\mathrm{CH}$ or diamond) used to maintain the material at high density. The shock is first launched in the ablator to then propagate in the material under study. When it 
reaches the material-CH interface, the impedance mismatch can induce a shock transmitted in the $\mathrm{CH}$ and a reflected shock returning in the material, which lies in a reshocked state until the return of the unloading wave.

Optical, fs laser pulses deposit their energy faster than the electron-phonon coupling timescale $(<1 \mathrm{ps})$. For semi-conductors such as $\mathrm{SiO}_{2}$, the laser photon energy (1.5 eV for a $800 \mathrm{~nm}$ titanium:sapphire laser) is smaller than the bandgap. Consequently, the sample is transparent at low intensity (below $10^{13} \mathrm{~W} / \mathrm{cm}^{2}$ ), but at intensities sufficient to induce nonlinear effects, absorption occurs through multiphoton ionization. In turn, the excited valence electrons interact with the ionized sample to bring it to temperatures of a few $\mathrm{eV}$. This process is known as non-thermal melting, because the electronic structure is lost before heating and melting of the sample. It was observed for the first time at a picosecond scale on germanium [257]. The sample was irradiated with a $100 \mathrm{fs}$ laser near $10^{13} \mathrm{~W} / \mathrm{cm}^{2}$ and probed with diffraction, using an $8 \mathrm{keV}$ laser-driven Copper K- $\alpha$ X-ray source. Non thermal melting was later observed with 100 fs resolution on InSb [258], where a Silicon K- $\alpha$ (1.8 $\mathrm{keV}$ ) source was used. The same experiment was replicated with betatron radiation [93] to demonstrate the ultrafast nature of the source, but the technique was not shown to be superior than with $\mathrm{K}-\alpha$ radiation.

Finally, XFEL fs X-ray pulses also drive matter to high temperatures in a unique way [229, 259, 260, 261, 262]. The short XFEL pulse ( $<100 \mathrm{fs})$ deposits its energy into the sample before hydrodynamic expansion can occur, but because of the much higher photon energies involved, XFEL light is primarily absorbed through photoionization of the atom's inner shells. The absorption depth (typically on the order of a few microns for mid-Z materials and for photon energies available at LCLS), permits isochoric heating of larger volumes than with optical radiation. Within a few femtoseconds, the core holes of the inner shells are refilled by electron transitions from the outer shells, through fluorescence or Auger decay. In the latter case, electrons with energies of a few hundred $\mathrm{eV}$ are emitted. Within a picosecond, the free electrons heat the lattice through phonon-electron coupling, leading to the production of warm dense matter. Local thermodynamic equilibrium (LTE) is achieved $\sim 1$ ps after irradiation, when electrons and ions have thermalized.

To study these electron-ion equilibration processes in warm dense matter, scientists use X-ray absorption spectroscopy techniques, such as X-ray absorption near edge structure (XANES), and extended X-ray absorption fine structure (EXAFS) [263, 264]. EXAFS has been developed on the OMEGA laser to diagnose iron up to $560 \mathrm{GPa}$ [265], and XANES at LULI-2000 to diagnose Mott nonmetal transitions [266] and the electronic structure of highly compressed $\mathrm{Al}$ [267]. XANES has also been used on table-top systems to characterize the phase transitions of $\mathrm{Al}$ up to a few $\mathrm{eV}$ [268, 269]. Models still need improvements in order to describe in detail the changes in the XANES spectra, mainly because the time resolution of these experiments was intrinsically limited by the X-ray probe duration. Ultrafast X-ray absorption experiments done at the Advanced Light Source synchrotron radiation facility have unraveled the electronic structure of warm dense Copper and Silicon dioxide [270, 271], but they required specific techniques to reduce the synchrotron pulse duration that are not practical or efficient. XANES experiments using the XFEL beam as a probe for shocked compressed matter were performed at LCLS-MEC near the Molybdenum LIII edge (2.520 $\mathrm{keV}$ ) [272], and the iron K-edge [273]. However, the stochastic nature of the LCLS SASE FEL spectrum makes this type of measurement extremely challenging, and since LCLS is 
used as a probe, it cannot be used as the pump, even in the two-color scheme, where pulses cannot be separated by more than 150 fs.

\subsection{Opacity in hot dense plasmas}

Opacity is the rate at which radiation is absorbed by matter. The structure and evolution of the Sun and stars depend on opacity, making it a cornerstone in HED physics. Although opacity has been studied for many decades [274, 275, 276, 277, 278, 279, 280], it is still among the most important issues in HED science because it affects nearly every HED experiment involving radiation. Although experiments can now recreate conditions that are similar in density and temperature to the conditions in the Sun's interior, they usually do not directly measure opacity over a broad X-ray energy range. They either measure the emission properties of the heated plasma and assume that emissivity and opacity are in equilibrium [281], or use non-tunable X-ray lasers [282]. Recent seminal experiments at Sandia's Z machine [238] measured the iron opacity at solar interior conditions higher than predicted at energies around $1 \mathrm{keV}$, which could explain discrepancies between solar models and helioseismic observations. This experiment used a broadband X-ray source that is sufficiently bright to overcome the thermal emission of the plasma itself. However, the rapid rate of ionization predicted by numerical modeling means that ideally the X-ray source for an opacity experiment should be a few tens of femtoseconds in duration to provide time-resolved information [283]. Once again, betatron X-ray radiation would be an ideal source for this application.

\subsection{Experimental techniques enabled by LWFA-driven sources}

We have discussed some physics where we believe LWFA light sources could have a significant impact. In the interest of applications, in this section we review whether LWFA light sources can be competitive for specific experimental techniques, or if progress needs to be achieved. Most of these applications require more photons than LWFA sources can presently provide, but we present some prospects on developing LWFA-driven light sources for three different techniques: imaging, absorption, and diffraction/scattering, which are illustrated in Figure 12 .

\subsubsection{Radiography and X-ray phase contrast imaging}

Betatron radiation and Compton scattering sources from LWFAs have both been used in proof-of-principle experiments for static X-ray phase contrast imaging (XPCI) of insects [192, 191], X-ray radiography of bone structure [94], and gamma-ray radiography of microchips [111].

The critical aspect of radiographing laser-driven shocks in HED or ICF relevant conditions $\left(10^{21}-10^{26} \mathrm{~cm}^{-3}\right.$ electron densities) is to have (i) high energy photons to penetrate dense plasmas, and (ii) enough photons to overcome the self emission of the plasma itself. At the NIF, 2D radiographic imaging of imploding capsules [254] is achieved with mid-Z backlighters (such as Zinc or Germanium). About $32 \mathrm{~kJ}$ or laser light is focused onto a foil, and typically conversion efficiencies on the order of $1 \%$ are obtained [285], which corresponds to $10^{13}-10^{14}$ photons $/ \mathrm{eV} / \mathrm{Sr}$ at $10 \mathrm{keV}$. The peak brightness of LWFA driven betatron and Compton 
a
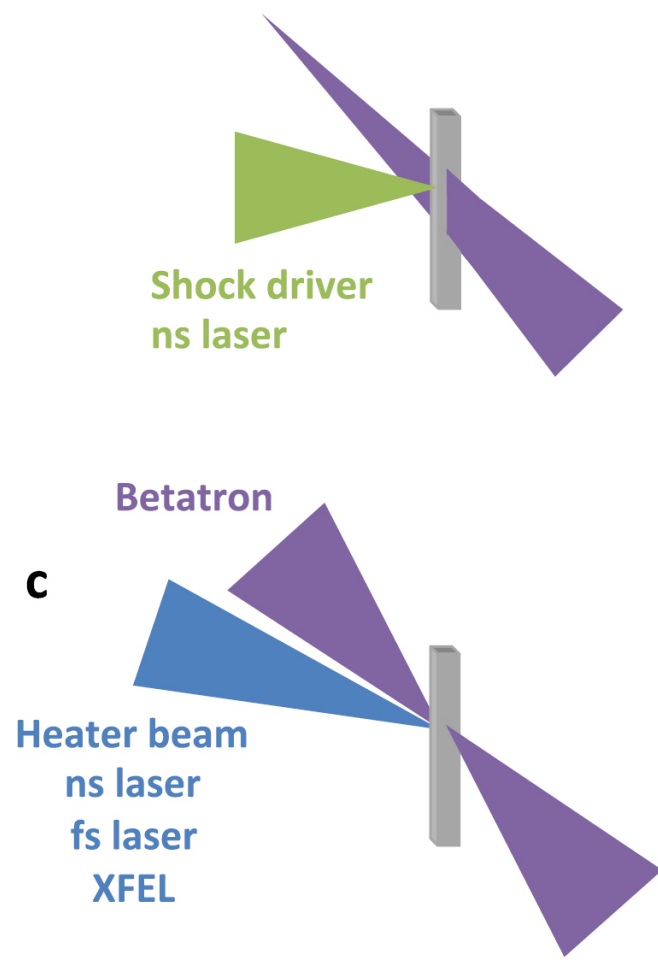

Heater beam

ns laser

fs laser

XFEL b
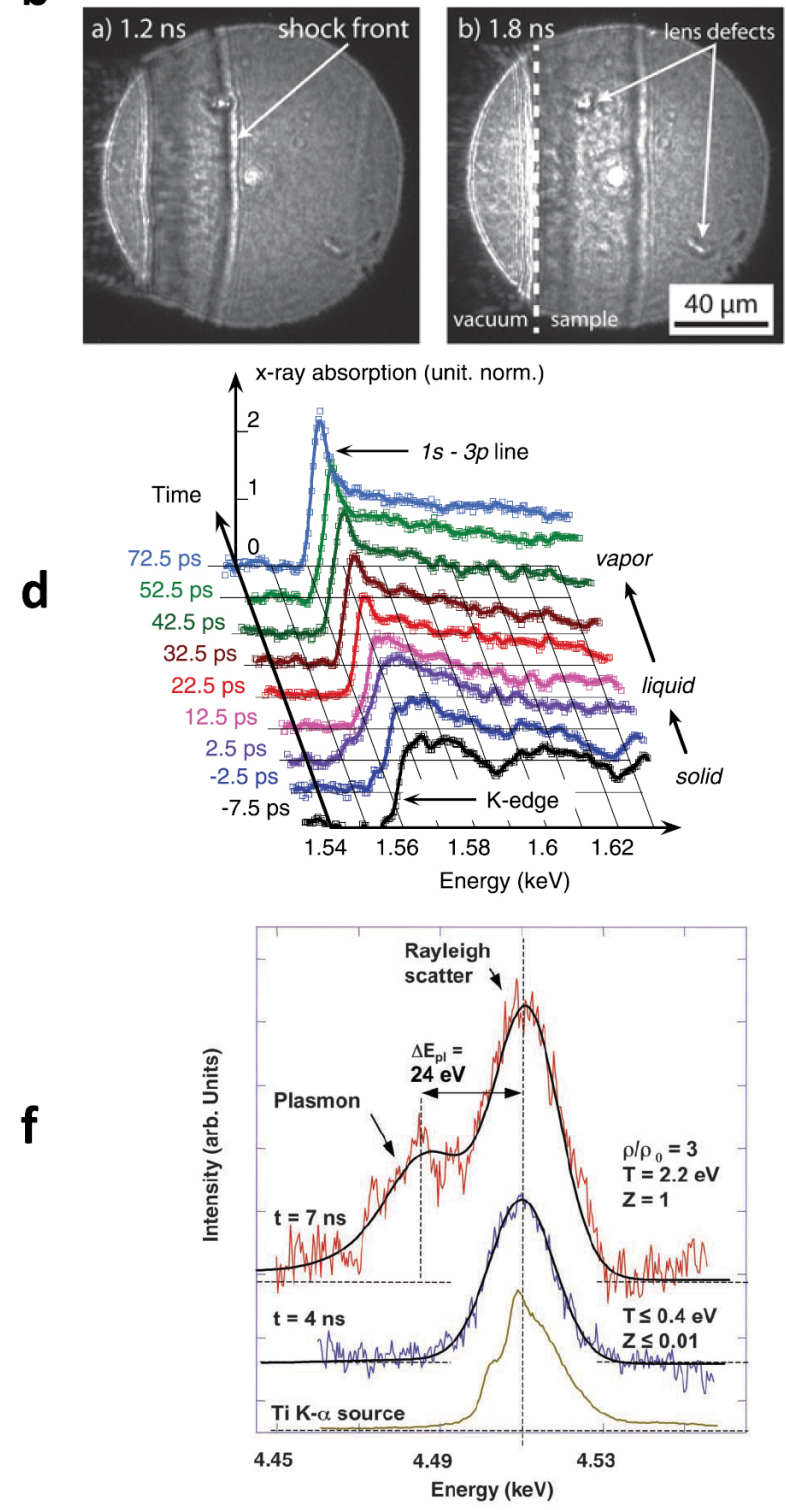

Figure 12: Illustration of possible $\mathrm{X}$-ray techniques using betatron radiation and Compton scattering for HED science. (a) radiography and phase contrast imaging of laser driven shocks, with examples of images (b) of laser-shocked diamond recently taken at the LCLS with the XFEL (from Schropp et al [256]). (c) X-ray absorption spectroscopy, with spectra (d) recorded at the $\mathrm{Al} \mathrm{K}$-edge $(1.5 \mathrm{keV})$ as a function of time after heating by a ps optical laser pulse, from Dorchies et al. [268] (e) X-ray diffraction and scattering, with the thomson scattering spectrum (f) of shock-compressed LiH, showing Rayleigh and inelastic plasmon scattering features. The probe is a Ti-K- $\alpha$ X-ray source. From Kritcher et al. [284] 
sources is 6 orders of magnitude higher than conventional backlighters because of their shorter duration (femtosecond vs. nanosecond). The current flux (photons/eV/Sr) of the source is sufficient to perform static single shot x-ray phase contrast imaging experiments, as suggested by recent experiments [191, 94, 192, 98]. Since the peak flux (photons/eV/s/Sr) of conventional and LWFA-driven backlighters are similar, the latter become a better option to look at transient $(<1 \mathrm{ps})$ phenomena.

XPCI, a topic we described in more details in a recent review paper [286], and also explained in Section 3, records the modifications of the phase of an X-ray beam as it passes through a material, as opposed to its amplitude recorded with conventional X-ray radiography techniques. It means that it is more sensitive to small density variations, and offers better contrast than conventional radiography. Although it is more desirable to have a monochromatic source for XPCI, the betatron source (in the blowout regime) is sufficiently small (a few microns) for this method. For a source size of $2 \mu \mathrm{m}$ and a critical energy of 8 $\mathrm{keV}$, the transverse coherence length of betatron radiation was measured to be $3 \mu \mathrm{m}, 5 \mathrm{~cm}$ away from the source, which is sufficient to observe Fresnel diffraction fringes [89]. XPCI appears to be a promising technique to radiograph implosion plasmas in ICF experiments [287], in order to obtain electron density information at the interfaces of materials such as DT ice/shell of a capsule. The requirement is to have a source sufficiently small $(<10 \mu \mathrm{m})$ to achieve good contrast.

\subsubsection{X-ray absorption spectroscopy}

A typical absorption spectrum of a material exhibits sharp edges, followed by EXAFS oscillations that are generally separated by a few eV and extend up to $\sim 200 \mathrm{eV}$ after the edge. Ideally, a XANES or EXAFS experiment should have a spectral resolution better than $2 \mathrm{eV}$. In general, the oscillation amplitude of the EXAFS signal is on the order of a few $\%$ of the total absorption signal (the edge step). Ideally, the random statistical noise, $S N=1 / \sqrt{N_{P h}}$, where $N_{P h}$ is the number of X-ray photons in the energy band of interest, should be 1/1000 of the EXAFS signal. This means that the condition $N_{P h}>10^{6} / \mathrm{eV}$ must be fulfilled to realize an EXAFS experiment with good statistics. Table 6 confirms these needs and reviews photon requirements for recent XANES and EXAFS time resolved experiments. Currently, state-of-the-art betatron radiation sources produce on the order of $10^{8}$ photons (in the full spectrum), and numbers of $10^{4}-10^{5}$ photons/eV have been reported around the $1.56 \mathrm{keV} \mathrm{Al} \mathrm{K-edge} \mathrm{[79]} \mathrm{and} \mathrm{measured} \mathrm{with} \mathrm{a} \mathrm{crystal} \mathrm{focusing} \mathrm{X-ray} \mathrm{spectrome-}$ ter. In view of Table 6 requirements, they are marginally competitive to execute EXAFS or XANES experiments, and should benefit from increased repetition rate laser systems. In addition to the quality of the X-ray source, instrumentation techniques (focusing, spectroscopy) will need to be specifically adapted for betatron X-ray radiation in order to achieve good spectral resolution. Recent experiments composed of Kirkpatrick-Baez (KB) mirrors and a flat KAP crystal (for betatron focusing and dispersion) have demonstrated the detection of about $10 \mathrm{X}$-ray photons within a $24 \mathrm{eV}$ window [288], which can be improved for practical applications. A gold-coated toroidal mirror with a grazing incidence angle of 2 degrees (70 $\%$ reflectivity at $1.5 \mathrm{keV}$ ) and an acceptance angle of $20 \mathrm{mrad}$ would, in principle, allow for about $10^{4}$ photons/eV near the $\mathrm{Al} \mathrm{K}$-edge to be refocused onto the target. This does not include filters that have to be placed in the beam path, or the efficiency of the (crystal or 
Table 6: Recent HED science XANES and EXAFS experiments and their photometrics

$\begin{array}{llllll}\text { Experiment } & \text { Edge } & \begin{array}{l}\text { Photon } \\ {[\mathrm{keV}]}\end{array} & \text { Energy } & \text { Resolution }[\mathbf{p s}] & \text { Photons } \\ \text { Saes 2003 [263] } & \mathrm{Ru}, \mathrm{L} 3, \mathrm{~L} 2 & 2.841,2.851 & 100 & 10^{6} / 0.1 \% \mathrm{BW} \\ \text { Ping 2013 [265] } & \mathrm{Fe}, \mathrm{K} & 7.1 & 120 & 10^{11} / \mathrm{eV} / \mathrm{Sr} \\ \text { Levy 2012 [266] } & \mathrm{Al}, \mathrm{K} & 1.56 & 3.5 & 10^{7} / \mathrm{eV} / \mathrm{Sr} \\ \text { Cho 2011 [270] } & \mathrm{Cu}, \mathrm{L} 3, & 0.932 & 2 & 10^{4} / \mathrm{eV} \\ \text { Harmand 2015[273] } & \mathrm{Fe}, \mathrm{K} & 7.1 & <1 & 10^{12} / \mathrm{eV}\end{array}$

grating) spectrometer used thereafter, which is typically a few percent.

Other features of absorption spectra that are of interest for HED science conditions are electronic shell absorption lines, which constitutes a first step toward benchmarking opacity models. The ionization state of a hot $(>100 \mathrm{eV})$ plasma can be determined by measuring its emission spectrum [229]. However, this technique does not provide time-resolved information on the ionization state as the electron and ion temperatures evolve. It could be achieved by looking at the transmission of betatron X-rays through the sample at different delays between the pump and the probe and at energies corresponding to a given electronic shell absorption lines. Since the plasma can reach very high temperatures, self emission can also be a problem for the measurement. In recent experiments, $\mathrm{K}$ shell emission from a $100 \mathrm{eV}$ Aluminum plasma produced $10^{6}-10^{7}$ photons $/ \mathrm{eV} / \mathrm{Sr}$ around $1.5 \mathrm{keV}$, which is slightly below the yield of current betatron X-ray sources at this energy. Once again, although the current source performance makes this application within reach, progress needs to be made on the instrumentation. Recent work shows that betatron X-rays can be focused with KirkpatrickBaez (KB) mirrors down to a $50 \mu \mathrm{m}$ size onto an Aluminum sample [288]. The configuration of this particular experiment would require several hundreds of shots to observe meaningful K-shell absorption features.

\subsubsection{X-ray diffraction and scattering}

$\mathrm{X}$-ray Thomson scattering is a widely used technique in HED experiments to measure the temperature, density and ionization states of a plasma [289, 284]. In X-ray Thomson scattering, a bright X-ray radiation with incident vector $\overrightarrow{k_{0}}$ irradiates a plasma and gets scattered (vector $\vec{k}_{s}$ ) at an angle $\theta$ with respect to $\overrightarrow{k_{0}}$. The angle $\theta$ determines the type of information that can be extracted. For example, the backscattered spectrum accesses the non-collective Compton scattering regime, while the forward scattered spectrum is sensitive to collective plasmon oscillations. For dense plasmas encountered in astrophysical or ICF conditions $\left(10^{21}-10^{26} \mathrm{~cm}^{-3}\right.$ electron densities), probes with energies above $10 \mathrm{keV}$ are needed. Because the scattering cross section $\left(6.65 \times 10^{-25} \mathrm{~cm}^{2}\right)$ is very small, a high number of photons is required. A minimum of $10^{12}$ photons at the sample is typical, which is at least 4 orders of magnitude above what betatron or Compton scattered X-rays from LWFAs can produce now. Additionally, a narrow bandwidth source is desired to be able to observe sharp features in the scattered spectrum. With this in mind, future LWFA-driven XFELs could be employed for this type of application.

X-ray diffraction is used in HED experiments because it can yield data on a crystal strain as a function of applied pressure (either static or laser-driven). The material strength prop- 
erties can be deduced from these measurements. The challenging aspect of such experiments is to obtain sufficiently bright X-ray backlighters with energies above $25 \mathrm{keV}$ to study high $\mathrm{Z}$ materials. White light (or Laue) X-ray diffraction uses a broadband X-ray source because the Bragg condition can be satisfied in multiple crystallographic planes in a single shot. Each plane results in a characteristic Laue spot, giving rise to a complex diffraction pattern. For dynamic compression experiments an implosion capsule X-ray source is typically used $[290,291]$, as well as layers of high Z-materials irradiated at intensities up to $10^{15} \mathrm{~W} / \mathrm{cm}^{2}$ [292]. In these experiments at least $10^{12}$ photons $/ \mathrm{eV} / \mathrm{Sr}$ is required. This number is also 3-4 orders of magnitude higher than the current performance of betatron X-ray sources.

\section{Conclusion and outlook}

In this paper, we have discussed potential applications of light sources driven by laserwakefield accelerators. We presented five sources: betatron X-ray radiation, Compton scattering radiation, bremsstrahlung, undulator radiation, and $\mathrm{THz}$ radiation. We have seen that they can enable significant applications in medicine, industry and defense, and high energy density science.

In this section, we summarize the performances of the sources, and their application space. A recent review paper presents a table of the relevant theoretical scaling parameters for betatron, Compton and undulator radiation, which is very useful to design an experiment for an application with particular requirements [5]. Here, in Table 7 we list the actual source parameters that have been experimentally reported (with best outcome for each source). We refer the reader to Section 2 for a description of each experiment and for theoretical properties and scaling laws of each source. Figure 13 shows the peak brightness of betatron, Compton and bremsstrahlung radiation from LWFA, and compares it with other conventional sources in the same energy range. In Table 8, we report all the applications listed in this paper, and indicate the most appropriate source for each. Betatron X-ray radiation, the most mature source, is emitted by electrons accelerated and wiggled by the wakefield, and is very similar to synchrotron radiation, with the following features: a broadband continuous spectrum (1$100 \mathrm{keV})$, a narrow divergence, a small source size $(\mu \mathrm{m})$, a short pulse duration (fs) and a perfect synchronization with the laser that produces it.

Applications that have already been demonstrated include X-ray phase contrast imaging of biological objects [191, 192, 98, 94] or HED plasmas, and time-resolved X-ray absorption spectroscopy. These should likely become routine applications for this source in a near future, where it can be coupled to high power and free electron lasers capable of driving matter to extreme states. Other techniques, such as scattering or diffraction in HED plasmas, will require at least 3 orders of magnitude more photons. Compton scattering is produced when relativistic LWFA electrons are wiggled in the field of a second laser pulse and emit Dopplerupshifted radiation. This mechanism possesses the same features as betatron radiation but, despite a small cross section, will be more efficient to produce photons beyond $100 \mathrm{keV}$. The highest photon energy reported to date in a LWFA-driven experiment is $18 \mathrm{MeV}$ [126]. It is also easier to tune than betatron radiation and can have a narrower bandwidth (provided the electron energy spread is small and the source operates in the linear regime where the 
scattering laser has a normalized vector potential $a_{0}<<1$ ). Hence, applications are naturally more geared toward gamma-ray radiography, photofission, and possibly nuclear resonance fluorescence. These have yet to be demonstrated.

For even higher photon energies, beyond $100 \mathrm{MeV}$, generation by bremsstrahlung becomes attractive. It is produced when LWFA electrons are converted to high energy photons in a high-Z material. Its source size ( typically $\sim 100 \mu \mathrm{m}$ ) is not yet as small as for Compton scattering ( potentially $\sim 1 \mu \mathrm{m}$ ), and thus improvements can be made (as discussed, 30 $\mu \mathrm{m}$ is reachable with current LWFA electron beams). This source has a sufficient number of photons to perform high resolution gamma-ray radiography [166], trigger giant dipole resonances [168], and photofission in natural Uranium [201].

An X-ray free electron laser based on LWFA has not yet been demonstrated, due to the stringent requirements on electron beam quality (emittance and energy spread) to achieve this. As of today, there has only been one demonstration of undulator radiation (with a conventional magnetic undulator) from a LWFA beam in the soft X-ray regime [161]. More details are given in Reference [5] on various schemes, scalings and designs. Projects are underway [162, 163], and if successful, this will be quite revolutionary, as the applications could be identical to a linac-based XFEL, but with a much more compact footprint. For example, the science realized during the first five years of operations at LCLS spans the domains of atomic, molecular, and optical physics; condensed matter physics; matter in extreme conditions; chemistry and soft matter, and biology [293]. Highlights include the first realization of an inner shell atomic x-ray laser [294], studies of protein structures [295, 296], lattice dynamics in compressed matter [297], surface bond breaking [298], and some of the hottest solid density plasmas created in the laboratory [229].

In a very different spectral range (about 5 orders of magnitude smaller in photon energy than X-rays), THz radiation from laser-wakefield accelerators has been demonstrated with sufficient energy per pulse [172] for possible applications in biomedical imaging, drug and explosives detection via spectroscopy, and ultrafast semiconductor studies. Currently, it is mostly used as a diagnostic of LWFA electron beams.

For cost and convenience, LWFA driven sources become especially attractive for short wavelengths. The construction cost of a large scale facility such as the APS synchrotron in the US (\$470 Millions), the LCLS XFEL in the US (\$380 Millions, with $\$ 300$ Millions saved by using the last kilometer of the existing linear accelerator), and the ELI-NP Compton gamma-ray source in Romania ( $\sim 300$ Millions) are prohibitive for universities or small laboratories. These facilities are accessible by users through regular calls for proposals, but the demand is usually very high. In comparison, a table-top setup for a betatron or Compton scattering source (including a $200 \mathrm{TW}$ commercial laser) costs on the order of $\$ 10$ Millions. $\mathrm{X}$-ray tubes, used for medical or industrial imaging, although much cheaper (up to $\$ 50 \mathrm{~K}$ for a ceramic tube), are not competitive for time-resolved studies. For longer wavelength sources such as THz radiation, LWFA sources may not be competitive. For example, a millimeter wave scanner used at airports costs about $\$ 180 \mathrm{~K}$. Most $\mathrm{THz}$ sources are already driven by visible or near infrared lasers, and the advent of $\mathrm{THz}$ quantum cascade lasers [299] have made $\mathrm{THz}$ sources a commercial, portable product.

In summary, we have given a comprehensive review of practical applications of light sources driven by laser-wakefield accelerators and discussed their feasibility, whether in the near future or with significant improvements. Most of the applications we have identified have 
Table 7: Summary of experimental X-ray and gamma-ray sources performance, with best achievements to date. These numbers are not necessarily reflective of the scaling laws, as each source was produced under different experimental conditions (laser parameters, electron density and energy). We refer to Section 2 for a detailed description of the theoretical and experimental properties of each source.

\begin{tabular}{|l|l|l|l|l|}
\hline & Betatron & Compton & Bremsstrahlung & FEL/Undulator \\
\hline Energy range (eV) & $10^{3}-10^{4}$ & $10^{3}-10^{7}$ & $10^{3}-10^{8}$ & 170 \\
Bandwidth (\%) & 100 & 50 & 100 & 22 \\
Number of Photons & $10^{9}$ & $10^{7}-10^{8}$ & $10^{8}$ & $7 \times 10^{4}$ \\
Brightness & $10^{24}$ & $10^{21}$ & $10^{17}-10^{21}$ & $10^{17}$ \\
Photons/eV & $10^{4}-10^{5}$ & $10^{2}-10^{3}$ & $10^{4}-10^{6}$ & $3 \times 10^{4}$ \\
Photons/eV/Sr & $2 \times 10^{9}$ & $10^{5}-10^{6}$ & $10^{2}-10^{4}$ & $10^{10}$ \\
Source size ( $\mu \mathrm{m})$ & 1.8 & 1.5 & 320 & 270 \\
$\begin{array}{l}\text { Duration (fs) } \\
\text { Collimation (mrad, }\end{array}$ & 2.5 & $<30$ & $<30$ & $<30$ \\
fwhm) & 10 & 50 & 0.18 \\
$\begin{array}{l}\text { Repetition rate } \\
(\mathrm{Hz})\end{array}$ & 10 & 10 & 10 & 10 \\
\hline
\end{tabular}

to be demonstrated, which we believe may be possible within 5 years for nuclear medicine, biomedical imaging ( $\mathrm{THz}$ ), backscattered inspection, photofission and opacity measurements in high energy density plasmas. Other applications, such as diffraction and scattering of HED plasmas, require a larger number of photons. As we discussed elsewhere [286], one current limit of such sources (compared to conventional accelerator technology) is their lower average flux, and their lack of reproducibility from shot to shot. These achievements, along with overcoming technological difficulties, should be made possible in the future thanks to developments in high repetition rate laser, diagnostic and target technology, to the emergence of new, petawatt-class short pulse laser facilities around the world [300] and higher repetition rate fiber laser technology [301].

\section{Acknowledgements}

Part of this work was performed under the auspices of the US Department of Energy under contract DE-AC52-07NA27344 at LLNL and supported by the Laboratory Directed Research and Development (LDRD) Program under tracking code 16-ERD-024 and 16-ERD-041. F.A. acknowledges support from the DOE Office of Fusion Energy Sciences under SCW1461. A.G.R.T acknowledges support from the NSF under CAREER Grant No. 1054164 and the Air Force Office of Scientific Research Young Investigator Program under award number FA9550-12-1-0310. 


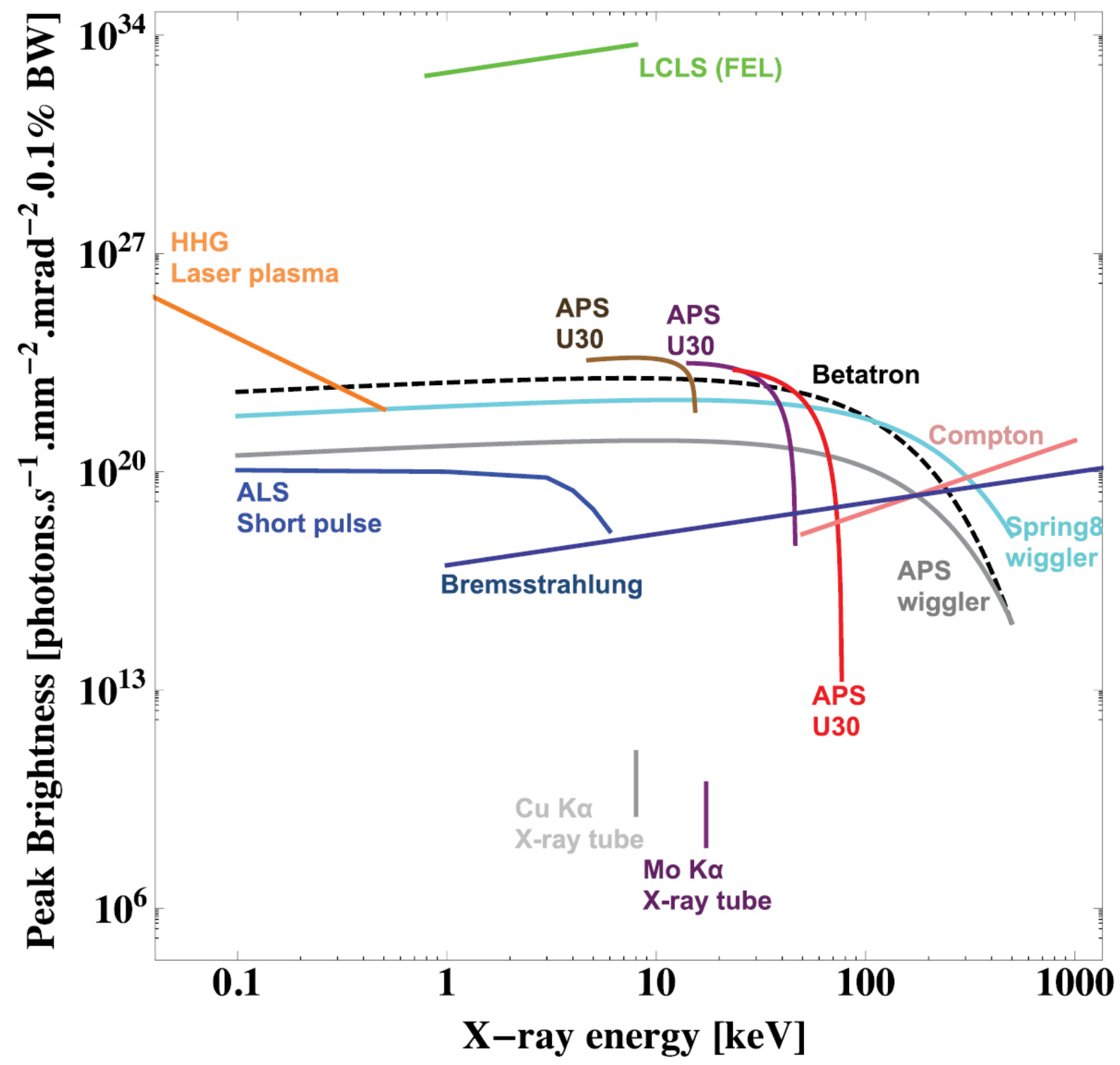

Figure 13: Peak brightness of betatron, Compton and bremsstrahlung radiation from LWFA compared to other types of sources in the same energy range. Sources included in this plot are: The APS synchrotron U30 undulator for harmonics 1, 3 and 5 (Argonne National Laboratory, USA), the ALS synchrotron (Lawrence Berkeley National Laboratory, USA), the Spring8 synchrotron (RIKEN, Japan), X-ray tubes (Copper and Molybdenum $\mathbf{K}_{\alpha}$ ), the LCLS free electron laser (SLAC, USA), and high harmonics generation from laser-produced plasmas. 
Table 8: Summary table for applications of light sources driven by laser wakefield accelerators. For each application discussed in this paper, we indicate whether the five LWFA light sources are a suitable choice. Demonstrated means that experiments have been performed, possible means that the current performance of the source allows for proof-of-principle demonstration. When needed, comments indicate what needs to be done in order to make the application possible, or if intrinsic properties of a given source are a disadvantage for the application.

\begin{tabular}{|c|c|c|c|c|c|}
\hline & Betatron & Compton & Bremsstrahlung & FEL/Undulator & $\mathrm{THz}$ \\
\hline $\begin{array}{l}\text { Diagnostic radiol- } \\
\text { ogy }\end{array}$ & $\begin{array}{l}\text { Demonstrated } \\
{[191,192,98,94]}\end{array}$ & Possible & $\begin{array}{l}\text { Less precise than } \\
\text { Compton and } \\
\text { Betatron (larger } \\
\text { spectral width } \\
\text { and source size) }\end{array}$ & $\begin{array}{l}\text { Possible if source } \\
\text { is demonstrated }\end{array}$ & No \\
\hline Nuclear medicine & No & Possible & Possible & No & No \\
\hline Biomedical/THz & No & No & No & No & Possible \\
\hline $\begin{array}{l}\text { Gamma-ray radio- } \\
\text { graphy }\end{array}$ & No & $\begin{array}{l}\text { Demonstrated } \\
{[111]}\end{array}$ & $\begin{array}{l}\text { Demonstrated } \\
{[166]}\end{array}$ & No & No \\
\hline $\begin{array}{l}\text { Backscattered in- } \\
\text { spection }\end{array}$ & Possible & Possible & $\begin{array}{l}\text { Less precise than } \\
\text { Compton and } \\
\text { Betatron (larger } \\
\text { spectral width) }\end{array}$ & $\begin{array}{l}\text { Possible if source } \\
\text { is demonstrated }\end{array}$ & No \\
\hline NRF & No & $\begin{array}{l}\text { Possible, but } \\
\text { need spectral } \\
\text { width }<10 \%\end{array}$ & $\begin{array}{l}\text { Likely impracti- } \\
\text { cal due to } 100 \% \\
\text { spectral width } \\
\text { and low LWFA } \\
\text { beam integrated } \\
\text { charge }\end{array}$ & No & No \\
\hline Photofission & No & Possible & $\begin{array}{l}\text { Demonstrated } \\
{[201]}\end{array}$ & No & No \\
\hline NEEC & No & No & No & $\begin{array}{l}\text { Unlikely in the } \\
\text { near future }\end{array}$ & No \\
\hline $\begin{array}{l}\text { Explosives and } \\
\text { drugs detection }\end{array}$ & No & No & No & & Possible \\
\hline $\begin{array}{l}\text { THz semiconduc- } \\
\text { tors study }\end{array}$ & No & No & No & No & Possible \\
\hline $\begin{array}{l}\text { XPCI/Radiography } \\
\text { of HED plasmas }\end{array}$ & Demonstrated $^{1}$ & Possible & Possible & $\begin{array}{l}\text { Possible if source } \\
\text { is demonstrated }\end{array}$ & \\
\hline XANES/EXAFS & Demonstrated $^{2}$ & Possible & No & $\begin{array}{l}\text { Too narrow spec- } \\
\text { tral width }\end{array}$ & No \\
\hline Opacity & $\begin{array}{l}\text { Possible, source } \\
\text { yield needs to be } \\
<\text { plasma emis- } \\
\text { sion }\end{array}$ & $\begin{array}{l}\text { Possible, source } \\
\text { yield needs to be } \\
<\text { plasma emis- } \\
\text { sion }\end{array}$ & $\begin{array}{l}\text { Possible, source } \\
\text { yield needs to } \\
\text { be < plasma } \\
\text { emission }\end{array}$ & $\begin{array}{l}\text { Limited band- } \\
\text { width }\end{array}$ & No \\
\hline $\begin{array}{l}\text { X-ray diffrac- } \\
\text { tion/scattering of } \\
\text { HED plasmas }\end{array}$ & $\begin{array}{l}3-4 \text { orders of } \\
\text { magnitude more } \\
\text { photons needed }\end{array}$ & $\begin{array}{l}3-4 \text { orders of } \\
\text { magnitude more } \\
\text { photons needed }\end{array}$ & $\begin{array}{l}\text { Not likely to be } \\
\text { more efficient } \\
\text { than } \mathrm{K}_{\alpha} \text { and di- } \\
\text { rect laser-driven } \\
\text { bremsstrahlung }\end{array}$ & No & No \\
\hline
\end{tabular}




\section{References}

[1] C. Chiu, M. Fomytskyi, F. Grigsby, F. Raischel, M. Downer, and T. Tajima. Laser electron accelerators for radiation medicine: a feasibility study. Medical Physics, 2003.

[2] S. V. Bulanov and V. S. Khoroshkov. Feasibility of using laser ion accelerators in proton therapy. Plasma Physics Reports, 28(5):453-456, 2002.

[3] P. Emma, R. Akre, J. Arthur, R. Bionta, C. Bostedt, J. Bozek, A. Brachmann, P. Bucksbaum, R. Coffee, F.-J. Decker, Y. Ding, D. Dowell, S. Edstrom, A. Fisher, J. Frisch, S. Gilevich, J. Hastings, G. Hays, Ph. Hering, Z. Huang, R. Iverson, H. Loos, M. Messerschmidt, A. Miahnahri, S. Moeller, H.-D. Nuhn, G. Pile, D. Ratner, J. Rzepiela, D. Schultz, T. Smith, P. Stefan, H. Tompkins, J. Turner, J. Welch, W. White, J. Wu, G. Yocky, and J. Galayda. First lasing and operation of an angstromwavelength free-electron laser. Nat. Photon., 4:641-647, 2011.

[4] E. Esarey, C.B. Schroeder, and W. P. Leemans. Physics of laser-driven plasma-based electron accelerators. Rev. Mod. Phys., 81:1229, 2009.

[5] S. Corde, K. Ta Phuoc, G. Lambert, R. Fitour, V. Malka, and A. Rousse. Femtosecond x rays from laser-plasma accelerators. Rev. Mod. Phys., 85:1-47, 2013.

[6] R. Paul Drake. High Energy Density Physics: Fundamentals, Inertial Fusion, and Experimental Astrophysics. 2006.

[7] T. Tajima and J. M. Dawson. Laser electron accelerator. Phys. Rev. Lett., 43:267-270, 1979.

[8] Donna Strickland and Gerard Mourou. Compression of amplified chirped optical pulses. Optics Communications, 56:219, 1985.

[9] C. E. Clayton, K.-C. Tzeng, D. Gordon, P. Muggli, W. B. Mori, C. Joshi, V. Malka, Z. Najmudin, A. Modena, D. Neely, and A. E. Dangor. Plasma wave generation in a self-focused channel of a relativistically intense laser pulse. Phys. Rev. Lett., 81:100, 1998.

[10] D. Gordon, K. C. Tzeng, C. E. Clayton, A. E. Dangor, V. Malka, K. A. Marsh, A. Modena, W. B. Mori, P. Muggli, Z. Najmudin, D. Neely, C. Danson, and C. Joshi. Observation of electron energies beyond the linear dephasing limit from a laser-excited relativistic plasma wave. Phys. Rev. Lett., 80:2133, 1998.

[11] A. Modena, Z. Najmudin, A. E. Dangor, C. E. Clayton, K. A. Marsh, C. Joshi, V. Malka, C. B. Darrow, C. Danson, D. Neely, and F. N. Walsh. Electron acceleration from the breaking of relativistic plasma waves. Nature, 377:606, 1995.

[12] M. I. K. Santala, Z. Najmudin, E. L. Clark, M. Tatarakis, K. Krushelnick, A. E. Dangor, V. Malka, J. Faure, R. Allott, and R. J. Clarke. Observation of a hot highcurrent electron beam from a self-modulated laser wakefield accelerator. Phys. Rev. Lett., 86:1277, 2001. 
[13] W.B. Mori. The physics of the nonlinear optics of plasmas at relativistic intensities for short-pulse lasers. Quantum Electronics, IEEE Journal of, 33(11):1942-1953, Nov 1997.

[14] C. Joshi, T. Tajima, J. M. Dawson, H. A. Baldis, and N. A. Ebrahim. Forward raman instability and electron acceleration. Phys. Rev. Lett., 47:1285-1288, Nov 1981.

[15] N. E. Andreev, V. I. Kirsanov, and L. M. Gorbunov. Stimulated processes and selfmodulation of a short intense laser pulse in the laser wakefield accelerator. Physics of Plasmas, 2(6), 1995.

[16] A. Ting, K. Krushelnick, C. I. Moore, H. R. Burris, E. Esarey, J. Krall, and P. Sprangle. Temporal evolution of self-modulated laser wakefields measured by coherent thomson scattering. Phys. Rev. Lett., 77:5377-5380, Dec 1996.

[17] R. Wagner, S.-Y. Chen, A. Maksimchuk, and D. Umstadter. Electron acceleration by a laser wakefield in a relativistically self-guided channel. Phys. Rev. Lett., 78:3125-3128, Apr 1997.

[18] K. Nakajima, D. Fisher, T. Kawakubo, H. Nakanishi, A. Ogata, Y. Kato, Y. Kitagawa, R. Kodama, K. Mima, H. Shiraga, K. Suzuki, K. Yamakawa, T. Zhang, Y. Sakawa, T. Shoji, Y. Nishida, N. Yugami, M. Downer, and T. Tajima. Observation of ultrahigh gradient electron acceleration by a self-modulated intense short laser pulse. Phys. Rev. Lett., 74:4428-4431, May 1995.

[19] C. A. Coverdale, C. B. Darrow, C. D. Decker, W. B. Mori, K-C. Tzeng, K. A. Marsh, C. E. Clayton, and C. Joshi. Propagation of intense subpicosecond laser pulses through underdense plasmas. Phys. Rev. Lett., 74:4659-4662, Jun 1995.

[20] Z. Najmudin, K. Krushelnick, E. L. Clark, S. P. D. Mangles, B. Walton, A. E. Dangor, S. Fritzler, V. Malka, E. Lefebvre, D. Gordon, F. S. Tsung, and C. Joshi. Selfmodulated wakefield and forced laser wakefield acceleration of electrons. Physics of Plasmas, 10(5), 2003.

[21] C. Gahn, G. D. Tsakiris, A. Pukhov, J. Meyer-ter Vehn, G. Pretzler, P. Thirolf, D. Habs, and K. J. Witte. Multi-mev electron beam generation by direct laser acceleration in high-density plasma channels. Phys. Rev. Lett., 83:4772-4775, Dec 1999.

[22] S. P. D. Mangles, B. R. Walton, M. Tzoufras, Z. Najmudin, R. J. Clarke, A. E. Dangor, R. G. Evans, S. Fritzler, A. Gopal, C. Hernandez-Gomez, W. B. Mori, W. Rozmus, M. Tatarakis, A. G. R. Thomas, F. S. Tsung, M. S. Wei, and K. Krushelnick. Electron acceleration in cavitated channels formed by a petawatt laser in low-density plasma. Phys. Rev. Lett., 94:245001, Jun 2005.

[23] A. Pukhov, Z.-M. Sheng, and J. Meyer-ter Vehn. Particle acceleration in relativistic laser channels. Physics of Plasmas (1994-present), 6(7), 1999. 
[24] Alexey V. Arefiev, Boris N. Breizman, Marius Schollmeier, and Vladimir N. Khudik. Parametric amplification of laser-driven electron acceleration in underdense plasma. Phys. Rev. Lett., 108:145004, Apr 2012.

[25] S. Kneip, S. R. Nagel, C. Bellei, N. Bourgeois, A. E. Dangor, A. Gopal, R. Heathcote, S. P. D. Mangles, J. R. Marquès, A. Maksimchuk, P. M. Nilson, K. Ta Phuoc, S. Reed, M. Tzoufras, F. S. Tsung, L. Willingale, W. B. Mori, A. Rousse, K. Krushelnick, and Z. Najmudin. Observation of synchrotron radiation from electrons accelerated in a petawatt-laser-generated plasma cavity. Phys. Rev. Lett., 100:105006, Mar 2008.

[26] S. P. D. Mangles, C. D. Murphy, Z. Najmudin, A. G. R. Thomas, J. L. Collier, A. E. Dangor, E. J. Divall, P. S. Foster, J. G. Gallacher, C. J. Hooker, D. A. Jaroszynski, A. J. Langley, W. B. Mori, P. A. Norreys, F. S. Tsung, R. Viskup, B. R. Walton, and K. Krushelnick. Monoenergetic beams of relativistic electrons from intense laserplasma interactions. Nature, 431:535-538, 2004.

[27] J. Faure, Y. Glinec, A. Pukhov, S. Kiselev, S. Gordienko, E. Lefebvre, J.-P. Rousseau, F. Burgy, and V. Malka. A laser-plasma accelerator producing monoenergetic electron beams. Nature, 431:541-544, 2004.

[28] C. G. R. Geddes, Cs. Toth, J. Van Tilborg, E. Esarey, C. B. Schroeder, D. Bruhwiler, C. Nieter, J. Cary, and W. P. Leemans. High-quality electron beams from a laser wakefield accelerator using plasma-channel guiding. Nature, 431:538-541, 2004.

[29] J. Faure, C. Rechatin, A. Norlin, A. Lifschitz, Y. Glinec, and V. Malka. Controlled injection and acceleration of electrons in plasma wakefields by colliding laser pulses. Nature, 444:737, 2006.

[30] S. P. D. Mangles, G. Genoud, M. S. Bloom, M. Burza, Z. Najmudin, A. Persson, K. Svensson, A. G. R. Thomas, and C.-G. Wahlström. Self-injection threshold in self-guided laser wakefield accelerators. Phys. Rev. ST Accel. Beams, 15:011302, Jan 2012.

[31] John M. Dawson. Particle simulation of plasmas. Rev. Mod. Phys., 55:403-447, Apr 1983.

[32] W. P. Leemans, B. Nagler, A. J. Gonsalves, Cs. Tth, K. Nakamura, C. G. R. Geddes, E. Esarey, C. B. Schroeder, and S. M. Hooker. Gev electron beams from a centimetrescale accelerator. Nat. Phys., 2:696, 2006.

[33] Nasr A. M. Hafz, Tae Moon Jeong, Il Woo Choi, Seong Ku Lee, Ki Hong Pae, Victor V. Kulagin, Jae Hee Sung, Tae Jun Yu, Kyung-Han Hong, Tomonao Hosokai, John R. Cary, Do-Kyeong Ko, and Jongmin Lee. Stable generation of gev-class electron beams from self-guided laserplasma channels. Nature Photonics, 2:571, 2008.

[34] C. E. Clayton, J. E. Ralph, F. Albert, R. A. Fonseca, S. H. Glenzer, C. Joshi, W. Lu, K. A. Marsh, S. F. Martins, W. B. Mori, A. Pak, F. S. Tsung, B. B. Pollock, J. S. Ross, L. O. Silva, and D. H. Froula. Self-guided laser wakefield acceleration beyond 1 gev using ionization-induced injection. Phys. Rev. Lett., 105:105003, 2010. 
[35] Hyung Taek Kim, Ki Hong Pae, Hyuk Jin Cha, I Jong Kim, Tae Jun Yu, Jae Hee Sung, Seong Ku Lee, Tae Moon Jeong, and Jongmin Leer. Enhancement of electron energy to the multi-gev regime by a dual-stage laser-wakefield accelerator pumped by petawatt laser pulses. Phys. Rev. Lett., 111:165002, 2013.

[36] X. Wang, R. Zgadzaj, N. Fazel, Z. Li, S. A. Yi, X. Zhang, W. Henderson, Y.-Y. Chang, R. Korzekwa, H.-E. Tsai, C.-H. Pai, H. Quevedo, G. Dyer, E. Gaul, M. Martinez, A. C. Bernstein, T. Borger, M. Spinks, M. Donovan, V. Khudik, G. Shvets, T. Ditmire, and M. C. Downer. Quasi-monoenergetic laser-plasma acceleration of electrons to 2 gev. Nat. Commun., doi:10.1038/ncomms2988, 2013.

[37] P. Leemans, W. J. Gonsalves, A. H.-S. Mao, K. Nakamura, C. Benedetti, B. Schroeder, C. Cs. Tóth, J. Daniels, E. Mittelberger, D. S. Bulanov, S. J.-L. Vay, G. $\tilde{R}$. Geddes, C. and E. Esarey. Multi-gev electron beams from capillary-discharge-guided subpetawatt laser pulses in the self-trapping regime. Phys. Rev. Lett., 113:245002, Dec 2014.

[38] A. Pukhov and J. Meyer-ter Vehn. Relativistic laser-plasma interaction by multidimensional particle-in-cell simulations. Physics of Plasmas (1994-present), 5(5), 1998.

[39] S. Gordienko and A. Pukhov. Scalings for ultrarelativistic laser plasmas and quasimonoenergetic electrons. Phys. Plasmas, 12(043109), 2005.

[40] W. Lu, M. Tzoufras, C. Joshi, F. S. Tsung, W. B. Mori, J. Vieira, R. A. Fonseca, and L. O. Silva. Generating multi-gev electron bunches using single stage laser wakefield acceleration in a 3d nonlinear regime. Phys. Rev. ST Acc. Beams, 10:061301, 2007.

[41] S P D Mangles, A G R Thomas, M C Kaluza, O Lundh, F Lindau, A Persson, Z Najmudin, C-G Wahlstr?m, C D Murphy, C Kamperidis, K L Lancaster, E Divall, and K Krushelnick. Effect of laser contrast ratio on electron beam stability in laser wakefield acceleration experiments. Plasma Physics and Controlled Fusion, 48(12B):B83, 2006.

[42] C. McGuffey, T. Matsuoka, S. Kneip, W. Schumaker, F. Dollar, C. Zulick, V. Chvykov, G. Kalintchenko, V. Yanovsky, A. Maksimchuk, A. G. R. Thomas, K. Krushelnick, and Z. Najmudin. Experimental laser wakefield acceleration scalings exceeding 100tw. Physics of Plasmas, 19(6), 2012.

[43] A. G. Khachatryan, F. A. van Goor, K. J. Boller, A. J. W. Reitsma, and D. A. Jaroszynski. Extremely short relativistic-electron-bunch generation in the laser wakefield via novel bunch injection scheme. Phys. Rev. Special Topics-Accelerators and Beams, 7(12), 2004.

[44] I. Kostyukov, E. Nerush, A. Pukhov, and V. Seredov. Electron self-injection in multidimensional relativistic-plasma wake fields. Phys. Rev. Lett., 103:175003, Oct 2009.

[45] D. H. Froula, C. E. Clayton, T. Döppner, K. A. Marsh, C. P. J. Barty, L. Divol, R. A. Fonseca, S. H. Glenzer, C. Joshi, W. Lu, S. F. Martins, P. Michel, W. B. Mori, J. P. Palastro, B. B. Pollock, A. Pak, J. E. Ralph, J. S. Ross, C. W. Siders, L. O. Silva, and 
T. Wang. Measurements of the critical power for self-injection of electrons in a laser wakefield accelerator. Phys. Rev. Lett., 103:215006, Nov 2009.

[46] A. G. R. Thomas. Scalings for radiation from plasma bubbles. Phys. Plasmas, 17(5):056708, 2010.

[47] C. Benedetti, C. B. Schroeder, E. Esarey, and W. P. Leemans. Plasma wakefields driven by an incoherent combination of laser pulses: A path towards high-average power laser-plasma acceleratorsa). Physics of Plasmas (1994-present), 21(5):-, 2014.

[48] S. Kalmykov, S. A. Yi, V. Khudik, and G. Shvets. Electron self-injection and trapping into an evolving plasma bubble. Phys. Rev. Lett., 103:135004, Sep 2009.

[49] C. B. Schroeder, E. Esarey, and B. A. Shadwick. Warm wave breaking of nonlinear plasma waves with arbitrary phase velocities. Phys. Rev. E, 72:055401, Nov 2005.

[50] E. Esarey, R. F. Hubbard, W. P. Leemans, A. Ting, and P. Sprangle. Electron injection into plasma wake fields by colliding laser pulses. Phys. Rev. Lett., 79:2682, 1997.

[51] D. Umstadter, J. K. Kim, and E. Dodd. Laser injection of ultrashort electron pulses into wakefield plasma waves. Phys. Rev. Lett., 76:2073-2076, Mar 1996.

[52] H. Kotaki, S. Masuda, M. Kando, J. K. Koga, and K. Nakajima. Head-on injection of a high quality electron beam by the interaction of two laser pulses. Physics of Plasmas (1994-present), 11(6), 2004.

[53] D. Kaganovich, A. Ting, D. F. Gordon, R. F. Hubbard, T. G. Jones, A. Zigler, and P. Sprangle. First demonstration of a staged all-optical laser wakefield acceleration. Physics of Plasmas (1994-present), 12(10):-, 2005.

[54] A. G. R. Thomas, C. D. Murphy, S. P. D. Mangles, A. E. Dangor, P. Foster, J. G. Gallacher, D. A. Jaroszynski, C. Kamperidis, K. L. Lancaster, P. A. Norreys, R. Viskup, K. Krushelnick, and Z. Najmudin. Monoenergetic electronic beam production using dual collinear laser pulses. Phys. Rev. Lett., 100:255002, Jun 2008.

[55] X. Davoine, E. Lefebvre, C. Rechatin, J. Faure, and V. Malka. Cold optical injection producing monoenergetic, multi-gev electron bunches. Phys. Rev. Lett., 102:065001, Feb 2009.

[56] R. Lehe, A. F. Lifschitz, X. Davoine, C. Thaury, and V. Malka. Optical transverse injection in laser-plasma acceleration. Phys. Rev. Lett., 111:085005, Aug 2013.

[57] J. Vieira, S. F. Martins, V. B. Pathak, R. A. Fonseca, W. B. Mori, and L. O. Silva. Magnetic control of particle injection in plasma based accelerators. Phys. Rev. Lett., 106:225001, May 2011.

[58] S. Bulanov, N. Naumova, F. Pegoraro, and J. Sakai. Particle injection into the wave acceleration phase due to nonlinear wake wave breaking. Phys. Rev. E, 58:R5257R5260, Nov 1998. 
[59] C. G. R. Geddes, K. Nakamura, G. R. Plateau, Cs. Toth, E. Cormier-Michel, E. Esarey, C. B. Schroeder, J. R. Cary, and W. P. Leemans. Plasma-density-gradient injection of low absolute-momentum-spread electron bunches. Phys. Rev. Lett., 100:215004, May 2008.

[60] K. Schmid, A. Buck, C. M. S. Sears, J. M. Mikhailova, R. Tautz, D. Herrmann, M. Geissler, F. Krausz, and L. Veisz. Density-transition based electron injector for laser driven wakefield accelerators. Phys. Rev. ST Accel. Beams, 13:091301, Sep 2010.

[61] A. J. Gonsalves, K. Nakamura, C. Lin, D. Panasenko, S. Shiraishi, T. Sokollik, C. Benedetti, C. B. Schroeder, C. G. R. Geddes, J. van Tilborg, J. Osterhoff, E. Esarey, C. Toth, and W. P. Leemans. Tunable laser plasma accelerator based on longitudinal density tailoring. Nat. Phys., 7:862, 2011.

[62] Z-H He, J A Nees, B Hou, K Krushelnick, and A G R Thomas. Enhancement of plasma wakefield generation and self-compression of femtosecond laser pulses by ionization gradients. Plasma Physics and Controlled Fusion, 56(8):084010, 2014.

[63] A. Buck, J. Wenz, J. Xu, K. Khrennikov, K. Schmid, M. Heigoldt, J. M. Mikhailova, M. Geissler, B. Shen, F. Krausz, S. Karsch, and L. Veisz. Shock-front injector for high-quality laser-plasma acceleration. Phys. Rev. Lett., 110:185006, May 2013.

[64] Min Chen, Zheng-Ming Sheng, Yan-Yun Ma, and Jie Zhang. Electron injection and trapping in a laser wakefield by field ionization to high-charge states of gases. Journal of Applied Physics, 99(5), 2006.

[65] A. Pak, K. A. Marsh, S. F. Martins, W. Lu, W. B. Mori, and C. Joshi. Injection and trapping of tunnel-ionized electrons into laser-produced wakes. Phys. Rev. Lett., 104:025003, 2010.

[66] C. McGuffey, A. G. R. Thomas, W. Schumaker, T. Matsuoka, V. Chvykov, F. J. Dollar, G. Kalintchenko, V. Yanovsky, A. Maksimchuk, K. Krushelnick, V. Yu. Bychenkov, I. V. Glazyrin, and A. V. Karpeev. Ionization induced trapping in a laser wakefield accelerator. Phys. Rev. Lett., 104:025003, 2010.

[67] B. Hidding, G. Pretzler, J. B. Rosenzweig, T. Königstein, D. Schiller, and D. L. Bruhwiler. Ultracold electron bunch generation via plasma photocathode emission and acceleration in a beam-driven plasma blowout. Phys. Rev. Lett., 108:035001, Jan 2012.

[68] B. B. Pollock, C. E. Clayton, J. E. Ralph, F. Albert, A. Davidson, L. Divol, C. Filip, S. H. Glenzer, K. Herpoldt, W. Lu, K. A. Marsh, J. Meinecke, W. B. Mori, A. Pak, T. C. Rensink, J. S. Ross, J. Shaw, G. R. Tynan, C. Joshi, and D. H. Froula. Demonstration of a narrow energy spread, 0,5 gev electron beam from a two-stage laser wakefield accelerator. Phys. Rev. Lett., 107:045001, 2011.

[69] J. S. Liu, C. Q. Xia, W. T. Wang, H. Y. Lu, Ch. Wang, A. H. Deng, W. T. Li, H. Zhang, X. Y. Liang, Y. X. Leng, X. M. Lu, C. Wang, J. Z. Wang, K. Nakajima, R. X. Li, and Z. Z. Xu. All-optical cascaded laser wakefield accelerator using ionization-induced injection. Phys. Rev. Lett., 107:035001, Jul 2011. 
[70] M. Chen, E. Esarey, C. B. Schroeder, C. G. R. Geddes, and W. P. Leemans. Theory of ionization-induced trapping in laser-plasma accelerators. Physics of Plasmas (1994present), 19(3):-, 2012.

[71] M. Vargas, W. Schumaker, Z.-H. He, Z. Zhao, K. Behm, V. Chvykov, B. Hou, K. Krushelnick, A. Maksimchuk, V. Yanovsky, and A. G. R. Thomas. Improvements to laser wakefield accelerated electron beam stability, divergence, and energy spread using three-dimensional printed two-stage gas cell targets. Applied Physics Letters, 104(17), 2014.

[72] S. Wang, C. E. Clayton, B. E. Blue, E. S. Dodd, K. A. Marsh, W. B. Mori, C. Joshi, S. Lee, P. Muggli, T. Katsouleas, F. J. Decker, M. J. Hogan, R. H. Iverson, P. Raimondi, D. Walz, R. Siemann, and R. Assmann. X-ray emission from betatron motion in a plasma wiggler. Phys. Rev. Lett., 88:135004, 2002.

[73] Antoine Rousse, Kim Ta Phuoc, Rahul Shah, Alexander Pukhov, Eric Lefebvre, Victor Malka, Sergey Kiselev, Frederic Burgy, Jean-Philippe Rousseau, Donald Umstadter, and Daniele Hulin. Production of a kev x-ray beam from synchrotron radiation in relativistic laser-plasma interaction. Phys. Rev. Lett., 93:135005, 2004.

[74] E. Esarey, B. A. Shadwick, P. Catravas, and W. P. Leemans. Synchrotron radiation from electron beams in plasma-focusing channels. Phys. Rev. E, 65:056505, 2002.

[75] I. Kostyukov, A. Pukhov, and S. Kiselev. Phenomenological theory of laser-plasma interaction in bubble regime. Physics of Plasmas, 11(11), 2004.

[76] W. Lu, C. Huang, M. Zhou, M. Tzoufras, F. S. Tsung, W. B. Mori, and T. Katsouleas. A nonlinear theory for multidimensional relativistic plasma wave wakefieldsa). Physics of Plasmas, 13(5), 2006.

[77] J.D. Jackson. Classical Electrodynamics. 1998.

[78] Kim Ta Phuoc, Sebastien Corde, Rahul Shah, Felicie Albert, Romuald Fitour, JeanPhilippe Rousseau, Frederic Burgy, Brigitte Mercier, and Antoine Rousse. Imaging electron trajectories in a laser-wakefield cavity using betatron x-ray radiation. Phys. Rev. Lett., 97:225002, 2006.

[79] Felicie Albert, Rahul Shah, Kim Ta Phuoc, Romuald Fitour, Frderic Burgy, JeanPhilippe Rousseau, Amar Tafzi, Denis Douillet, Thierry Lefrou, and Antoine Rousse. Betatron oscillations of electrons accelerated in laser wakefields characterized by spectral x-ray analysis. Phys. Rev. E, 77:056402, 2008.

[80] G. R. Plateau, C. G. R. Geddes, D. B. Thorn, M. Chen, C. Benedetti, E. Esarey, A. J. Gonsalves, N. H. Matlis, K. Nakamura, C. B. Schroeder, S. Shiraishi, T. Sokollik, J. Van Tilborg, Cs. Toth, S. Trotsenko, T. S. Kim, M. Battaglia, Th. Stohlker, and W. P. Leemans. Low-emittance electron bunches from a laser-plasma accelerator measured using single-shot x-ray spectroscopy. Phys. Rev. Lett., 109:064802, 2012. 
[81] I Kostyukov, S. Kiselev, and A. Pukhov. X-ray generation in an ion channel. Phys. Plasmas, 10:4818, 2003.

[82] Károly Németh, Baifei Shen, Yuelin Li, Hairong Shang, Robert Crowell, Katherine C. Harkay, and John R. Cary. Laser-driven coherent betatron oscillation in a laserwakefield cavity. Phys. Rev. Lett., 100:095002, Mar 2008.

[83] S. Kiselev, A. Pukhov, and I. Kostyukov. X-ray generation in strongly nonlinear plasma waves. Phys. Rev. Lett., 93:135004, Sep 2004.

[84] F. Albert, B.B. Pollock, J.L Shaw, K. A. Marsh, J. E. Ralph, Y. H. Chen, D. Alessi, A. Pak, C. E. Clayton, S. H. Glenzer, and C. Joshi. Angular dependence of betatron x-ray spectra from a laser wakefield accelerator. Phys. Rev. Lett., 111:235004, 2013.

[85] A. G. R. Thomas and K. Krushelnick. Betatron x-ray generation from electrons accelerated in a plasma cavity in the presence of laser fields. Phys. Plasmas, 16(10):103103, 2009.

[86] J.L. Martins, S.F. Martins, R.A. Fonseca, and L.O. Silva. Radiation post-processing in pic codes. In Antoine Rousse Dino A. Jaroszynski, editor, Harnessing Relativistic Plasma waves as Novel Radiation Sources from Terahertz to X-rays and Beyond, volume 7359 of Proc. SPIE, pages 73590V-1-73590V-8, 2009.

[87] J. L. Martins, S. F. Martins, R. A. Fonseca, and L. O. Silva. Radiation in 1.5 gev and 12 gev laser wakefield acceleration stages from pic simulations. AIP Conference Proceedings, 1299(1), 2010.

[88] J.L. Martins, S.F. Martins, R.A. Fonseca, and L.O. Silva. X-ray modeling in laserwakefield accelerators. Plasma Science, IEEE Transactions on, 39(11):2826-2827, Nov 2011.

[89] S. Kneip, C. McGuffey, J. L. Martins, S. F. Martins, C. Bellei, V. Chvykov, F. Dollar, R. Fonseca, C. Huntington, G. Kalintchenko, A. Maksimchuk, S. P. D. Mangles, T. Matsuoka, S. R. Nagel, C. A. J. Palmer, J. Schreiber, K. Ta Phuoc, A. G. R. Thomas, V. Yanovsky, L. O. Silva, K. Krushelnick, and Z. Najmudin. Bright spatially coherent synchrotron x-rays from a table-top source. Nat. Phys., 6:980-983, 2010.

[90] S. Fourmaux, S. Corde, K. Ta Phuoc, P. M. Leguay, S. Payeur, P. Lassonde, S. Gnedyuk, G. Lebrun, C. Fourment, V. Malka, S. Sebban, A. Rousse, and J. C. Kieffer. Demonstration of the synchrotron-type spectrum of laser-produced betatron radiation. New J. Phys., 13:033017, 2011.

[91] M. Schnell, A. Savert, I. Uschmann, M. Reuter, M. Nicolai, T. Kampfer, B. Landgraf, O. Jackel, O. Jansen, A. Pukhov, M. C. Kaluza, and C. Spielmann. Optical control of hard x-ray polarization by electron injection in a laser wakefield accelerator. Nat. Commun, 4:2421, 2013. 
[92] Silvia Cipiccia, Mohammad R. Islam, Bernhard Ersfeld, Richard P. Shanks, Enrico Brunetti, Gregory Vieux, Xue Yang, Riju C. Issac, Samuel M.Wiggins, Gregor H.Welsh, Maria-Pia Anania, Dzmitry Maneuski, Rachel Montgomery, Gary Smith, Matthias Hoek, David J. Hamilton, Nuno R. C. Lemos, Dan Symes, Pattathil P. Rajeev, Val O. Shea, Joo M. Dias, and Dino A. Jaroszynski. Gamma-rays from harmonically resonant betatron oscillations in a plasma wake. Nat. Phys., 7:867, 2011.

[93] K. Ta Phuoc, R. Fitour, A. Tafzi, T. Garl, N. Artemiev, R. Shah, F. Albert, D. Boschetto, A. Rousse, D-E. Kim, A. Pukhov, V. Seredov, and I. Kostyukov. Demonstration of the ultrafast nature of laser produced betatron radiation. Phys. Plasmas, 14:080701, 2007.

[94] J. M. Cole, J. C. Wood, N. C. Lopes, K. Poder, R. L. Abel, S. Alatabi, J. S. J. Bryant, A. Jin, S. Kneip, K. Mecseki, D. R. Symes, S. P. D. Mangles, and Z. Najmudin. Laserwakefield accelerators as hard x-ray sources for $3 \mathrm{~d}$ medical imaging of human bone. Scientific Reports, 5:13244 EP -, 082015.

[95] M. Schnell, A Savert, B. Landgraf, M. Reuter, M. Nicolai, O. Jackel, C. Peth, T. Thiele, O. Jansen, A. Pukhov, O. Willi, M. C. Kaluza, and C. Spielmann. Deducing the electron-beam diameter in a laser-plasma accelerator using x-ray betatron radiation. Phys. Rev. Lett., 108:075001, 2012.

[96] J. Ju, K. Svensson, A. Dpp, H. E. Ferrari, K. Cassou, O. Neveu, G. Genoud, F. Wojda, M. Burza, A. Persson, O. Lundh, C.-G. Wahlstrm, and B. Cros. Enhancement of xrays generated by a guided laser wakefield accelerator inside capillary tubes. Applied Physics Letters, 100(19), 2012.

[97] S. Chen, N. D. Powers, I. Ghebregziabher, C. M. Maharjan, C. Liu, G. Golovin, S. Banerjee, J. Zhang, N. Cunningham, A. Moorti, S. Clarke, S. Pozzi, and D. P. Umstadter. Mev-energy x rays from inverse compton scattering with laser-wakefield accelerated electrons. Phys. Rev, Lett., 110:155003, 2013.

[98] J. Wenz, S. Schleede, K. Khrennikov, M. Bech, P. Thibault, M. Heigoldt, F. Pfeiffer, and S. Karsch. Quantitative x-ray phase-contrast microtomography from a compact laser-driven betatron source. Nat Commun, 6, 072015.

[99] D. B. Thorn, C. G. R. Geddes, N. H. Matlis, G. R. Plateau, E. H. Esarey, M. Battaglia, C. B. Schroeder, S. Shiraishi, Th. Sthlker, C. Tth, and W. P. Leemans. Spectroscopy of betatron radiation emitted from laser-produced wakefield accelerated electronsa). Review of Scientific Instruments, 81(10), 2010.

[100] C. Thaury, E. Guillaume, S. Corde, R. Lehe, M. Le Bouteiller, K. Ta Phuoc, X. Davoine, J. M. Rax, A. Rousse, and V. Malka. Angular-momentum evolution in laser-plasma accelerators. Phys. Rev. Lett., 111:135002, 2013.

[101] F Albert, B B Pollock, J L Shaw, K A Marsh, J E Ralph, Y-H Chen, D Alessi, A Pak, C E Clayton, S H Glenzer, and C Joshi. Measuring the angular dependence of betatron 
x-ray spectra in a laser-wakefield accelerator. Plasma Physics and Controlled Fusion, 56(8):084016, 2014.

[102] S. Corde, C. Thaury, A. Lifschitz, G. Lambert, K. Ta Phuoc, X. Davoine, R. Lehe, D. Douillet, A. Rousse, and V. Malka. Observation of longitudinal and transverse self-injections in laser-plasma accelerators. Nat. Comm., 4:1501, 2013.

[103] R. C. Shah, F. Albert, K. Ta Phuoc, O. Shevchenko, D. Boschetto, A. Pukhov, S. Kiselev, F. Burgy, J.-P. Rousseau, and A. Rousse. Coherence-based transverse measurement of synchrotron x-ray radiation from relativistic laser-plasma interaction and laseraccelerated electrons. Phys. Rev. E, 74:045401(R), 2006.

[104] S. Kneip, C. McGuffey, J. L. Martins, M. S. Bloom, V. Chvykov, F. Dollar, R. Fonseca, S. Jolly, G. Kalintchenko, K. Krushelnick, A. Maksimchuk, S. P. D. Mangles, Z. Najmudin, C. A. J. Palmer, K. Ta Phuoc, W. Schumaker, L. O. Silva, J. Vieira, V. Yanovsky, and A. G. R. Thomas. Characterization of transverse beam emittance of electrons from a laser-plasma wakefield accelerator in the bubble regime using betatron x-ray radiation. Phys. Rev. ST Accel. Beams, 15:021302, 2012.

[105] S. Corde, C. Thaury, K. Ta Phuoc, A. Lifschitz, G. Lambert, J. Faure, O. Lundh, E. Benveniste, A. Ben-Ismail, L. Arantchuk, A. Marciniak, A. Stordeur, P. Brijesh, A. Rousse, A. Specka, and V. Malka. Mapping the x-ray emission region in a laserplasma accelerator. Phys. Rev. Lett., 107:215004, Nov 2011.

[106] J Vieira, S F Martins, F Fiuza, C K Huang, W B Mori, S P D Mangles, S Kneip, $\mathrm{S}$ Nagel, Z Najmudin, and L O Silva. Influence of realistic parameters on state-of-theart laser wakefield accelerator experiments. Plasma Physics and Controlled Fusion, 54(5):055010, 2012.

[107] S. Corde, K. Ta Phuoc, R. Fitour, J. Faure, A. Tafzi, J. P. Goddet, V. Malka, and A. Rousse. Controlled betatron x-ray radiation from tunable optically injected electrons. Phys. Rev. Lett., 107:255003, Dec 2011.

[108] A. Popp, J. Vieira, J. Osterhoff, Zs. Major, R. Hrlein, M. Fuchs, R. Weingartner, T. P. Rowlands-Rees, M. Marti, R. A. Fonseca, S. F. Martins, L. O. Silva, S. M. Hooker, F. Krausz, F. Gruner, and S. Karsch. All-optical steering of laser-wakefield-accelerated electron beams. Phys. Rev. Lett., 105:215001, 2010.

[109] S. P. D. Mangles, G. Genoud, S. Kneip, M. Burza, K. Cassou, B. Cros, N. P. Dover, C. Kamperidis, Z. Najmudin, A. Persson, J. Schreiber, F. Wojda, and C.-G. Wahlstrm. Controlling the spectrum of x-rays generated in a laser-plasma accelerator by tailoring the laser wavefront. Applied Physics Letters, 95(18):-, 2009.

[110] A. H. Compton. Phys. Rev., 21:483, 1923.

[111] K. Ta Phuoc, S. Corde, C. Thaury, V. Malka, A. Tafzi, J. P. Goddet, R. C. Shah, S. Sebban, and A. Rousse. All-optical compton gamma-ray source. Nature Photonics, 6:308-311, 2012. 
[112] Eric Esarey, Sally K. Ride, and Phillip Sprangle. Nonlinear thomson scattering of intense laser pulses from beams and plasmas. Phys. Rev. E, 48:3003-3021, Oct 1993.

[113] F. Mackenroth and A. Di Piazza. Nonlinear compton scattering in ultrashort laser pulses. Phys. Rev. A, 83:032106, Mar 2011.

[114] Y. Y. Lau, F He, D. P. Umstadter, and R. Kowalczyk. Nonlinear thomson scattering: A tutorial. Phys. Plasmas, 10(5, Part 2):2155-2162, 2003.

[115] D. Seipt and B. Kämpfer. Nonlinear compton scattering of ultrashort intense laser pulses. Phys. Rev. A, 83:022101, Feb 2011.

[116] F. V. Hartemann and A. K. Kerman. Classical theory of nonlinear compton scattering. Phys. Rev. Lett., 76:624-627, Jan 1996.

[117] F. Albert, S. G. Anderson, D. J. Gibson, R. A. Marsh, C. W. Siders, C. P. J. Barty, and F. V. Hartemann. Three-dimensional theory of weakly nonlinear compton scattering. Physics of Plasmas (1994-present), 18(1):-, 2011.

[118] F. V. Hartemann, D. J. Gibson, W. J. Brown, A. Rousse, K. Ta Phuoc, V. Mallka, J. Faure, and A. Pukhov. Compton scattering x-ray sources driven by laser wakefield acceleration. Phys. Rev. ST Accel. Beams, 10:011301, Jan 2007.

[119] Isaac Ghebregziabher, B. A. Shadwick, and Donald Umstadter. Spectral bandwidth reduction of thomson scattered light by pulse chirping. Phys. Rev. ST Accel. Beams, 16:030705, Mar 2013.

[120] A. G. R Thomas, C. P. Ridgers, S. S. Bulanov, B. J. Griffin, and S. P. D. Mangles. Strong radiation-damping effects in a gamma-ray source generated by the interaction of a high-intensity laser with a wakefield-accelerated electron beam. Phys. Rev. X, 2:041004, 2012.

[121] H. Schwoerer, B. Liesfeld, H.-P. Schlenvoigt, K.-U. Amthor, and R. Sauerbrey. Thomson-backscattered $\mathrm{x}$ rays from laser-accelerated electrons. Phys. Rev. Lett., 96:014802, Jan 2006.

[122] Hai-En Tsai, Xiaoming Wang, Joseph M. Shaw, Zhengyan Li, Alexey V. Arefiev, Xi Zhang, Rafal Zgadzaj, Watson Henderson, V. Khudik, G. Shvets, and M. C. Downer. Compact tunable compton x-ray source from laser-plasma accelerator and plasma mirror. Physics of Plasmas, 22(2), 2015.

[123] N. D. Powers, I. Ghebregziabher, G. Golovin, C. Liu, S. Chen, S. Banerjee, J. Zhang, and D. P. Umstadter. Nat. Photon., 10.1038/nphoton.2013.314, 2013.

[124] Cheng Liu, Grigory Golovin, Shouyuan Chen, Jun Zhang, Baozhen Zhao, Daniel Haden, Sudeep Banerjee, Jack Silano, Hugon Karwowski, and Donald Umstadter. Generation of 9 mev $\gamma$-rays by all-laser-driven compton scattering with second-harmonic laser light. Opt. Lett., 39(14):4132-4135, Jul 2014. 
[125] K. Khrennikov, J. Wenz, A. Buck, J. Xu, M. Heigoldt, L. Veisz, and S. Karsch. Tunable all-optical quasimonochromatic thomson x-ray source in the nonlinear regime. Phys. Rev. Lett., 114:195003, May 2015.

[126] G. Sarri, J. Corvan, D. W. Schumaker, M. Cole, J. A. Di Piazza, H. Ahmed, C. Harvey, H. Keitel, C. K. Krushelnick, P.D̃. Mangles, S. Z. Najmudin, D. Symes, G.R. Thomas, A. M. Yeung, Z. Zhao, and M. Zepf. Ultrahigh brilliance multi-mev $\gamma$-ray beams from nonlinear relativistic thomson scattering. Phys. Rev. Lett., 113:224801, Nov 2014.

[127] J. Ballam, G. B. Chadwick, R. Gearhart, Z. G. T. Guiragossian, P. R. Klein, A. Levy, M. Menke, J. J. Murray, P. Seyboth, G. Wolf, C. K. Sinclair, H. H. Bingham, W. B. Fretter, K. C. Moffeit, W. J. Podolsky, M. S. Rabin, A. H. Rosenfeld, and R. Windmolders. Total and partial photoproduction cross sections at 1.44, 2.8, and 4.7 gev. Phys. Rev. Lett., 23:498-501, Sep 1969.

[128] L. Casano, A. Marino, G. Matone, M. Roccella, C. Schaerf, and A. Tranquilli. Production of a Beam of Polarized and Monochromatic gamma Rays by Compton Scattering of Laser Light Against High-Energy Electrons. Laser and Unconventional Optics J., 1974.

[129] Eli nuclear physics. http://www.eli-np.ro.

[130] Cameron G.R. Geddes, Sergey Rykovanov, Nicholas H. Matlis, Sven Steinke, Jean-Luc Vay, Eric H. Esarey, Bernhard Ludewigt, Kei Nakamura, Brian J. Quiter, Carl B. Schroeder, Csaba Toth, and Wim P. Leemans. Compact quasi-monoenergetic photon sources from laser-plasma accelerators for nuclear detection and characterization. $\mathrm{Nu}$ clear Instruments and Methods in Physics Research Section B: Beam Interactions with Materials and Atoms, 350:116 - 121, 2015.

[131] Henry R. Weller, Mohammad W. Ahmed, Haiyan Gao, Werner Tornow, Ying K. Wu, Moshe Gai, and Rory Miskimen. Research opportunities at the upgraded HIgammaS facility. Prog. Part. Nucl. Phys., 62:257-303, 2009.

[132] David J. Gibson, Scott G. Anderson, Christopher P. J. Barty, Shawn M. Betts, Rex Booth, Winthrop J. Brown, John K. Crane, Robert R. Cross, David N. Fittinghoff, Fred V. Hartemann, Jaroslav Kuba, Gregory P. Le Sage, Dennis R. Slaughter, Aaron M. Tremaine, Alan J. Wootton, Edward P. Hartouni, Paul T. Springer, and James B. Rosenzweig. Pleiades: A picosecond compton scattering x-ray source for advanced backlighting and time-resolved material studies. Physics of Plasmas, 11(5), 2004.

[133] F. Albert, S.G. Anderson, G.A. Anderson, S.M. Betts, D.G. Gibson, C.A. Hagmann, J. Hall, M.S. Johnson, M.J. Messerly, V.A. Semenov, M.Y. Shverdin, A.M. Tremaine, F.V. Hartemann, C.W. Siders, D.P. McNabb, and C.P.J. Barty. Isotope-specific detection of low-density materials with laser-based monoenergetic gamma-rays. Optics Letters, 35:354, 2010. 
[134] K Aoki, K Hosono, T Hadame, H Munenaga, K Kinoshita, M Toda, S Amano, S Miyamoto, T Mochizuki, M Aoki, and D Li. High-energy photon beam production with laser-compton backscattering. Nuclear Instruments and Methods in Physics Research Section A: Accelerators, Spectrometers, Detectors and Associated Equipment, 516(23):228 - 236, 2004.

[135] A. Variola. The thomx project status. In Proceedings of IPAC2014, Dresden, Germany, 2014.

[136] A. Jochmann, A. Irman, M. Bussmann, J. P. Couperus, T. E. Cowan, A. D. Debus, M. Kuntzsch, K. W. D. Ledingham, U. Lehnert, R. Sauerbrey, H. P. Schlenvoigt, D. Seipt, Th. Stöhlker, D. B. Thorn, S. Trotsenko, A. Wagner, and U. Schramm. High resolution energy-angle correlation measurement of hard $\mathrm{x}$ rays from laser-thomson backscattering. Phys. Rev. Lett., 111:114803, Sep 2013.

[137] A. Di Piazza, C. Müller, K. Z. Hatsagortsyan, and C. H. Keitel. Extremely highintensity laser interactions with fundamental quantum systems. Rev. Mod. Phys., 84:1177-1228, Aug 2012.

[138] Richard T. Hammond. Radiation reaction at ultrahigh intensities. Phys. Rev. A, 81:062104, Jun 2010.

[139] L. D. Landau and E. M. Lifshitz. The Classical Theory of Fields. Pergamon, Oxford, 1975.

[140] A. Di Piazza, K. Z. Hatsagortsyan, and C. H. Keitel. Quantum radiation reaction effects in multiphoton compton scattering. Phys. Rev. Lett., 105:220403, Nov 2010.

[141] Igor V. Sokolov, Natalia M. Naumova, John A. Nees, and Gérard A. Mourou. Pair creation in qed-strong pulsed laser fields interacting with electron beams. Phys. Rev. Lett., 105:195005, Nov 2010.

[142] J Koga, T. Z. Esirkepov, and S. V. Bulanov. Nonlinear thomson scattering in the strong radiation damping regime. Phys. Plasmas, 12(9), 2005.

[143] A. Di Piazza, K. Z. Hatsagortsyan, and C. H. Keitel. Strong signatures of radiation reaction below the radiation-dominated regime. Phys. Rev. Lett., 102:254802, Jun 2009.

[144] M. Chen, E. Esarey, C. G. R. Geddes, C. B. Schroeder, G. R. Plateau, S. S. Bulanov, S. Rykovanov, and W. P. Leemans. Modeling classical and quantum radiation from laser-plasma accelerators. Phys. Rev. ST Accel. Beams, 16:030701, Mar 2013.

[145] S. S. Bulanov, C. B. Schroeder, E. Esarey, and W. P. Leemans. Electromagnetic cascade in high-energy electron, positron, and photon interactions with intense laser pulses. Phys. Rev. A, 87:062110, Jun 2013.

[146] N. Neitz and A. Di Piazza. Stochasticity effects in quantum radiation reaction. Phys. Rev. Lett., 111:054802, Aug 2013. 
[147] M. Vranic, L. Martins, J. J. Vieira, A. Fonseca, R. and O. Silva, L. All-optical radiation reaction at $10^{21} \mathrm{~W} / \mathrm{cm}^{2}$. Phys. Rev. Lett., 113:134801, Sep 2014.

[148] A. Zhidkov, S. Masuda, S. Bulanov, S. J. Koga, T. Hosokai, and R. Kodama. Radiation reaction effects in cascade scattering of intense, tightly focused laser pulses by relativistic electrons: Classical approach. Phys. Rev. ST Accel. Beams, 17:054001, May 2014.

[149] Jian-Xing Li, Karen Z. Hatsagortsyan, and Christoph H. Keitel. Robust signatures of quantum radiation reaction in focused ultrashort laser pulses. Phys. Rev. Lett., 113:044801, Jul 2014.

[150] T. G. Blackburn, C. P. Ridgers, J. G. Kirk, and A. R. Bell. Quantum radiation reaction in laser electron-beam collisions. Phys. Rev. Lett., 112:015001, Jan 2014.

[151] DL Burke, RC Field, G HortonSmith, JE Spencer, D Walz, SC Berridge, WM Bugg, K Shmakov, AW Weidemann, C Bula, KT McDonald, EJ Prebys, C Bamber, SJ Boege, T Koffas, T Kotseroglou, AC Melissinos, DD Meyerhofer, DA Reis, and W Raggk. Positron production in multiphoton light-by-light scattering. Phys. Rev. Lett., 79(9):1626-1629, 1997.

[152] F. V. Hartemann, C. W. Siders, and C. P. J. Barty. Compton scattering in ignited thermonuclear plasmas. Phys. Rev. Lett., 100:125001, Mar 2008.

[153] Zhirong Huang and Kwang-Je Kim. Review of x-ray free-electron laser theory. Phys. Rev. ST Accel. Beams, 10:034801, Mar 2007.

[154] A..G. R. Thomas. Algorithm for calculating spectral intensity due to charged particles in arbitrary motion. Phys. Rev. ST Accel. Beams, 13(2):020702, 2010.

[155] Zhirong Huang, Yuantao Ding, and Carl B. Schroeder. Compact x-ray free-electron laser from a laser-plasma accelerator using a transverse-gradient undulator. Phys. Rev. Lett., 109:204801, Nov 2012.

[156] A. R. Maier, A. Meseck, S. Reiche, C. B. Schroeder, T. Seggebrock, and F. Grüner. Demonstration scheme for a laser-plasma-driven free-electron laser. Phys. Rev. X, 2:031019, Sep 2012.

[157] T. Seggebrock, A. R. Maier, I. Dornmair, and F. Grüner. Bunch decompression for laser-plasma driven free-electron laser demonstration schemes. Phys. Rev. ST Accel. Beams, 16:070703, Jul 2013.

[158] F. J. Grüner, C. B. Schroeder, A. R. Maier, S. Becker, and J. M. Mikhailova. Spacecharge effects in ultrahigh current electron bunches generated by laser-plasma accelerators. Phys. Rev. ST Accel. Beams, 12:020701, Feb 2009.

[159] C. B. Schroeder, E. Esarey, and W. P. Leemans. Electron-beam conditioning by thomson scattering. Phys. Rev. Lett., 93:194801, Nov 2004. 
[160] H. P. Schlenvoigt, K. Haupt, A. Debus, F. Budde, O. Jackel, S. Pfotenhauer, H. Schwoerer, E. Rohwer, J. G. Gallacher, E. Brunetti, R. P. Shanks, S. M. Wiggins, and D. A. Jaroszynski. A compact synchrotron radiation source driven by a laser-plasma wakefield accelerator. Nat Phys, 4(2):130-133, 022008.

[161] Matthias Fuchs, Raphael Weingartner, Antonia Popp, Zsuzsanna Major, Stefan Becker, Jens Osterhoff, Isabella Cortrie, Benno Zeitler, Rainer Horlein, George D. Tsakiris, Ulrich Schramm, Tom P. Rowlands-Rees, Simon M. Hooker, Dietrich Habs, Ferenc Krausz, Stefan Karsch, and Florian Gruner. Laser-driven soft-x-ray undulator source. Nat Phys, 5(11):826-829, 112009.

[162] M.E. Courpie. The lunex5 project. In Proceedings of FEL2012, Nara, Japan, 2012.

[163] C. B. Schroeder, C. Benedetti, E. Esarey, Leemans W., J. Van Tilborg, Y. T. Ding, Z. Huang, F. Gruner, and Maier A. R. Applications of laser plasma accelerator beams to free electron lasers. In Proceedings of FEL2012, Nara, Japan, 2012.

[164] H. A. Bethe and L. C. Maximon. Theory of bremsstrahlung and pair production. i. differential cross section. Phys. Rev., 93:768-784, Feb 1954.

[165] R. D. Edwards, M. A. Sinclair, T. J. Goldsack, K. Krushelnick, F. N. Beg, E. L. Clark, A. E. Dangor, Z. Najmudin, M. Tatarakis, B. Walton, M. Zepf, K. W. D. Ledingham, I. Spencer, P. A. Norreys, R. J. Clarke, R. Kodama, Y. Toyama, and M. Tampo. Characterization of a gamma-ray source based on a laser-plasma accelerator with applications to radiography. Applied Physics Letters, 80(12), 2002.

[166] Y. Glinec, J. Faure, L. Le Dain, S. Darbon, T. Hosokai, J. J. Santos, E. Lefebvre, J. P. Rousseau, F. Burgy, B. Mercier, and V. Malka. High-resolution gamma-ray radiography produced by a laser-plasma driven electron source. Phys. Rev. Lett., 94:025003, Jan 2005.

[167] A. Ben-Ismail, J. Faure, and V. Malka. Optimization of gamma-ray beams produced by a laser-plasma accelerator. Nuclear Instruments and Methods in Physics Research Section A: Accelerators, Spectrometers, Detectors and Associated Equipment, 629(1):382 $-386,2011$.

[168] A. Giulietti, N. Bourgeois, T. Ceccotti, X. Davoine, S. Dobosz, P. D’Oliveira, M. Galimberti, J. Galy, A. Gamucci, D. Giulietti, L. A. Gizzi, D. J. Hamilton, E. Lefebvre, L. Labate, J. R. Marquès, P. Monot, H. Popescu, F. Réau, G. Sarri, P. Tomassini, and $\mathrm{P}$. Martin. Intense gamma ray source in the giant-dipole-resonance range driven by 10-tw laser pulses. Phys. Rev. Lett., 101:105002, Sep 2008.

[169] Yuji Oishi, Takuya Nayuki, Alexei Zhidkov, Takashi Fujii, and Koshichi Nemoto. Evaluation of yields of $\gamma$-rays produced by electrons from gas jets irradiated by low-energy laser pulses: Towards virtual radioisotopes. Japanese Journal of Applied Physics, 50(4R):042702, 2011. 
[170] S. Cipiccia, S. M. Wiggins, R. P. Shanks, M. R. Islam, G. Vieux, R. C. Issac, E. Brunetti, B. Ersfeld, G. H. Welsh, M. P. Anania, D. Maneuski, N. R. C. Lemos, R. A. Bendoyro, P. P. Rajeev, P. Foster, N. Bourgeois, T. P. A. Ibbotson, P. A. Walker, V. O. Shea, J. M. Dias, and D. A. Jaroszynski. A tuneable ultra-compact high-power, ultra-short pulsed, bright gamma-ray source based on bremsstrahlung radiation from laser-plasma accelerated electrons. Journal of Applied Physics, 111(6):-, 2012.

[171] C. B. Schroeder, E. Esarey, J. van Tilborg, and W. P. Leemans. Theory of coherent transition radiation generated at a plasma-vacuum interface. Phys. Rev. E, 69:016501, Jan 2004.

[172] W. P. Leemans, C. G. R. Geddes, J. Faure, Cs. Tóth, J. van Tilborg, C. B. Schroeder, E. Esarey, G. Fubiani, D. Auerbach, B. Marcelis, M. A. Carnahan, R. A. Kaindl, J. Byrd, and M. C. Martin. Observation of terahertz emission from a laser-plasma accelerated electron bunch crossing a plasma-vacuum boundary. Phys. Rev. Lett., 91:074802, Aug 2003.

[173] S. I. Bajlekov, M. Heigoldt, A. Popp, J. Wenz, K. Khrennikov, S. Karsch, and S. M. Hooker. Longitudinal electron bunch profile reconstruction by performing phase retrieval on coherent transition radiation spectra. Phys. Rev. ST Accel. Beams, 16:040701, Apr 2013.

[174] A. D. Debus, M. Bussmann, U. Schramm, R. Sauerbrey, C. D. Murphy, Zs. Major, R. Hörlein, L. Veisz, K. Schmid, J. Schreiber, K. Witte, S. P. Jamison, J. G. Gallacher, D. A. Jaroszynski, M. C. Kaluza, B. Hidding, S. Kiselev, R. Heathcote, P. S. Foster, D. Neely, E. J. Divall, C. J. Hooker, J. M. Smith, K. Ertel, A. J. Langley, P. Norreys, J. L. Collier, and S. Karsch. Electron bunch length measurements from laser-accelerated electrons using single-shot thz time-domain interferometry. Phys. Rev. Lett., 104:084802, Feb 2010.

[175] J. van Tilborg, C. B. Schroeder, C. V. Filip, Cs. Tóth, C. G. R. Geddes, G. Fubiani, R. Huber, R. A. Kaindl, E. Esarey, and W. P. Leemans. Temporal characterization of femtosecond laser-plasma-accelerated electron bunches using terahertz radiation. Phys. Rev. Lett., 96:014801, Jan 2006.

[176] J. van Tilborg, C. B. Schroeder, C. V. Filip, Cs. Toth, C. G. R. Geddes, G. Fubiani, E. Esarey, and W. P. Leemans. Terahertz radiation as a bunch diagnostic for laserwakefield-accelerated electron bunches. Physics of Plasmas (1994-present), 13(5):-, 2006.

[177] J. van Tilborg, Cs. Tóth, N. H. Matlis, G. R. Plateau, and W. P. Leemans. Singleshot measurement of the spectral envelope of broad-bandwidth terahertz pulses from femtosecond electron bunches. Opt. Lett., 33(11):1186-1188, Jun 2008.

[178] J. van Tilborg, C. B. Schroeder, Cs. Tóth, C. G. R. Geddes, E. Esarey, and W. P. Leemans. Single-shot spatiotemporal measurements of high-field terahertz pulses. Opt. Lett., 32(3):313-315, Feb 2007. 
[179] Y. Glinec, J. Faure, A. Norlin, A. Pukhov, and V. Malka. Observation of fine structures in laser-driven electron beams using coherent transition radiation. Phys. Rev. Lett., 98:194801, May 2007.

[180] O. Lundh, J. Lim, C. Rechatin, L. Ammoura, A. Ben-Ismal, X. Davoine, G. Gallot, J-P. Goddet, E. Lefebvre, V. Malka, and J. Faure. Few femtosecond, few kiloampere electron bunch produced by a laserplasma accelerator. Nat. Phys., 7:219-222, 2011.

[181] C. Lin, J. van Tilborg, K. Nakamura, A. J. Gonsalves, N. H. Matlis, T. Sokollik, S. Shiraishi, J. Osterhoff, C. Benedetti, C. B. Schroeder, Cs. Tóth, E. Esarey, and W. P. Leemans. Long-range persistence of femtosecond modulations on laser-plasmaaccelerated electron beams. Phys. Rev. Lett., 108:094801, Mar 2012.

[182] Herb Brody. Medical imaging. Nature, 502(7473):S81-S81, 102013.

[183] Nist xcom photon cross section database. http://www.nist.gov/pml/data/xcom/.

[184] Martin Hoheisel. Review of medical imaging with emphasis on x-ray detectors. Nuclear Instruments and Methods in Physics Research Section A: Accelerators, Spectrometers, Detectors and Associated Equipment, 563(1):215 - 224, 2006. Proceedings of the 7th International Workshop on Radiation Imaging DetectorsIWORID 20057th International Workshop on Radiation Imaging Detectors.

[185] TJ Davos, D Gao, TE Gureyev, AW Stevenson, and SW Wilkins. Phase-contrast imaging of weakly absorbing materials using hard x-rays. Nature, 373(6515):595-598, Feb 161995.

[186] S.W. Wilkins, T. E. Gureyev, D. Gao, A. Pogany, and A. W. Stevenson. Phase contrast imaging using polychromatic hard x-rays. Nature, 384:335, 1996.

[187] R A Lewis. Medical phase contrast x-ray imaging: current status and future prospects. Physics in Medicine and Biology, 49(16):3573, 2004.

[188] Atsushi Momose. Recent advances in x-ray phase imaging. Japanese Journal of Applied Physics, 44(9A):6355-6367, 2005.

[189] John J Socha, Mark W Westneat, Jon F Harrison, James S Waters, and Wah-Keat Lee. Real-time phase-contrast x-ray imaging: a new technique for the study of animal form and function. BMC Biology, 5(6):1-15, 2007.

[190] A. Momose. Phase-sensitive imaging and phase tomography using x-ray interferometers. Optics Express, 19(3):2303, 2013.

[191] S. Fourmaux, S. Corde, K. Ta Phuoc, P. Lassonde, G. Lebrun, S. Payeur, F. Martin, S. Sebban, V. Malka, A. Rousse, and J. C. Kieffer. Single shot phase contrast imaging using laser-produced betatron x-ray beams. Optics Letters, 36:2426, 2011. 
[192] S. Kneip, C. McGuffey, F. Dollar, M. S. Bloom, V. Chvykov, G. Kalintchenko, K. Krushelnick, A. Maksimchuk, S. P. D. Mangles, T. Matsuoka, Z. Najmudin, C. A. J. Palmer, J. Schreiber, W. Schumaker, A. G. R. Thomas, and V. Yanovsky. X-ray phase contrast imaging of biological specimens with femtosecond pulses of betatron radiation from a compact laser plasma wakefield accelerator. Appl. Phys. Lett., 99:093701, 2011.

[193] Z. Najmudin, S. Kneip, M. S. Bloom, S. P. D. Mangles, O. Chekhlov, A. E. Dangor, A. Döpp, K. Ertel, S. J. Hawkes, J. Holloway, C. J. Hooker, J. Jiang, N. C. Lopes, H. Nakamura, P. A. Norreys, P. P. Rajeev, C. Russo, M. J. V. Streeter, D. R. Symes, and M. Wing. Compact laser accelerators for x-ray phase-contrast imaging. Philosophical Transactions of the Royal Society of London A: Mathematical, Physical and Engineering Sciences, 372(2010), 2014.

[194] P. Oliva, M. Carpinelli, B. Golosio, P. Delogu, M. Endrizzi, J. Park, I. Pogorelsky, V. Yakimenko, O. Williams, and J. Rosenzweig. Quantitative evaluation of single-shot inline phase contrast imaging using an inverse compton x-ray source. Applied Physics Letters, 97(13), 2010.

[195] Frank E. Carroll. Tunable monochromatic x rays: A new paradigm in medicine. American Journal of Roentgenology, 179(3):583-590, 2014/12/30 2002.

[196] Richard Van Noorden. The medical testing crisis. NATURE, 504(7479):202-204, DEC 122013.

[197] K W D Ledingham, P McKenna, T McCanny, S Shimizu, J M Yang, L Robson, J Zweit, J M Gillies, J Bailey, G N Chimon, R J Clarke, D Neely, P A Norreys, J L Collier, R P Singhal, M S Wei, S P D Mangles, P Nilson, K Krushelnick, and M Zepf. High power laser production of short-lived isotopes for positron emission tomography. Journal of Physics D: Applied Physics, 37(16):2341, 2004.

[198] Roman A. Schmitt and Nathan Sugarman. Uranium photofission yields. Phys. Rev., 95:1260-1267, Sep 1954.

[199] D. Habs and U Koster. Production of medical radioisotopes with high specific activity in photonuclear reactions with gamma-beams of high intensity and large brilliance. Appl. Phys. B, 103:501-519, 2011.

[200] D. Habs, T. Tajima, J. Schreiber, C. P.J. Barty, M. Fujiwara, and P. G. Thirolf. Vision of nuclear physics with photo-nuclear reactions by laser-driven $\gamma$ beams. The European Physical Journal D, 55(2):279-285, 2009.

[201] S. A. Reed, V. Chvykov, G. Kalintchenko, T. Matsuoka, P. Rousseau, V. Yanovsky, C. R. Vane, J. R. Beene, D. Stracener, D. R. Schultz, and A. Maksimchuk. Photonuclear fission with quasimonoenergetic electron beams from laser wakefields. Applied Physics Letters, 89(23), 2006.

[202] W. Schumaker, G. Sarri, M. Vargas, Z. Zhao, K. Behm, V. Chvykov, B. Dromey, B. Hou, A. Maksimchuk, J. Nees, V. Yanovsky, M. Zepf, A. G. R. Thomas, and 
K. Krushelnick. Measurements of high-energy radiation generation from laser-wakefield accelerated electron beams. Physics of Plasmas, 21(5), 2014.

[203] W. P. Leemans, D. Rodgers, P. E. Catravas, C. G. R. Geddes, G. Fubiani, E. Esarey, B. A. Shadwick, R. Donahue, and A. Smith. Gamma-neutron activation experiments using laser wakefield accelerators. Physics of Plasmas, 8(5), 2001.

[204] E Pickwell and V P Wallace. Biomedical applications of terahertz technology. Journal of Physics D: Applied Physics, 39(17):R301, 2006.

[205] Bradley Ferguson, Shaohong Wang, Doug Gray, Derek Abbot, and X.-C. Zhang. T-ray computed tomography. Opt. Lett., 27(15):1312-1314, Aug 2002.

[206] P. Reimers, J. Goebbels, H.-P. Weise, and K. Wilding. Some aspects of industrial non-destructive evaluation by $\mathrm{x}$ - and gamma-ray computed tomography. Nuclear Instruments and Methods in Physics Research, 221(1):201 - 206, 1984. Proceedings of the International Workshop on X- and -Ray Imaging Techniques.

[207] S. C. Wilks, W. L. Kruer, M. Tabak, and A. B. Langdon. Absorption of ultra-intense laser pulses. Phys. Rev. Lett., 69:1383-1386, Aug 1992.

[208] Paul Gibbon. Efficient production of fast electrons from femtosecond laser interaction with solid targets. Phys. Rev. Lett., 73:664-667, Aug 1994.

[209] G. Malka and J. L. Miquel. Experimental confirmation of ponderomotive-force electrons produced by an ultrarelativistic laser pulse on a solid target. Phys. Rev. Lett., 77:75-78, Jul 1996.

[210] M. D. Perry, J. A. Sefcik, T. Cowan, S. Hatchett, A. Hunt, M. Moran, D. Pennington, R. Snavely, and S. C. Wilks. Hard x-ray production from high intensity laser solid interactions (invited). Review of Scientific Instruments, 70(1), 1999.

[211] C. Courtois, R. Edwards, A. Compant La Fontaine, C. Aedy, S. Bazzoli, J. L. Bourgade, J. Gazave, J. M. Lagrange, O. Landoas, L. Le Dain, D. Mastrosimone, N. Pichoff, G. Pien, and C. Stoeckl. Characterisation of a mev bremsstrahlung x-ray source produced from a high intensity laser for high areal density object radiography. Physics of Plasmas, 20(8), 2013.

[212] A. Ben-Ismail, O. Lundh, C. Rechatin, J. K. Lim, J. Faure, S. Corde, and V. Malka. Compact and high-quality gamma-ray source applied to $10 \mathrm{~m}$-range resolution radiography. Applied Physics Letters, 98(26):-, 2011.

[213] Sudeep Banerjee, Shouyuan Chen, Nathan Powers, Daniel Haden, Cheng Liu, G. Golovin, Jun Zhang, Baozhen Zhao, S. Clarke, S. Pozzi, J. Silano, H. Karwowski, and Donald Umstadter. Compact source of narrowband and tunable x-rays for radiography. Nuclear Instruments and Methods in Physics Research Section B: Beam Interactions with Materials and Atoms, 350:106 - 111, 2015. 
[214] G Zentai. X-ray imaging for homeland security. International Journal of Signal and Imaging Systems Engineering, 3(1):13-20, 2010.

[215] F. Albert, S.G. Anderson, G.A. Anderson, S.M. Betts, D.G. Gibson, C.A. Hagmann, J. Hall, M.S. Johnson, M.J. Messerly, V.A. Semenov, M.Y. Shverdin, A.M. Tremaine, F.V. Hartemann, C.W. Siders, D.P. McNabb, and C.P.J. Barty. Characterization and applications of a tunable, laser-based, Mev-class compton-scattering $\gamma$-ray source. Phys. Rev. ST Accel. Beams, 13:070704, 2010.

[216] H. Kuwabara, Y. Mori, and Y. Kitagawa. Standoff detection of hidden objects using backscattered ultra-intense laser-produced x-rays. Journal of Applied Physics, 114(8):, 2013.

[217] U. Kneissl, H.H. Pitz, and A. Zilges. Investigation of nuclear structure by resonance fluorescence scattering. Progress in Particle and Nuclear Physics, 37:349 - 433, 1996.

[218] H. Metzger. ?investigation of nuclear structure by resonance fluorescence scattering. Progress in Nuclear Physics, 7, 1959.

[219] William Bertozzi, Stephen E. Korbly, Robert J. Ledoux, and William Park. Nuclear resonance fluorescence and effective $\mathrm{z}$ determination applied to detection and imaging of special nuclear material, explosives, toxic substances and contraband. Nuclear Instruments and Methods in Physics Research Section B: Beam Interactions with Materials and Atoms, 261(1-2):331 - 336, 2007. The Application of Accelerators in Research and Industry, Proceedings of the Nineteenth International Conference on The Application of Accelerators in Research and Industry: Nineteenth International Conference on The Application of Accelerators in Research and Industry.

[220] W. Bertozzi, J. A. Caggiano, W. K. Hensley, M. S. Johnson, S. E. Korbly, R. J. Ledoux, D. P. McNabb, E. B. Norman, W. H. Park, and G. A. Warren. Nuclear resonance fluorescence excitations near $2 \mathrm{mev}$ in ${ }^{235} \mathrm{U}$ and ${ }^{239} \mathrm{Pu}$. Phys. Rev. C, 78:041601, Oct 2008.

[221] Nobuhiro Kikuzawa, Ryoichi Hajima, Nobuyuki Nishimori, Eisuke Minehara, Takehito Hayakawa, Toshiyuki Shizuma, Hiroyuki Toyokawa, and Hideaki Ohgaki. Nondestructive detection of heavily shielded materials by using nuclear resonance fluorescence with a laser-compton scattering -ray source. Applied Physics Express, 2(3):036502, 2009.

[222] C. A. Hagmann, J. M. Hall, M. S. Johnson, D. P. McNabb, J. H. Kelley, C. Huibregtse, E. Kwan, G. Rusev, and A. P. Tonchev. Transmission-based detection of nuclides with nuclear resonance fluorescence using a quasimonoenergetic photon source. Journal of Applied Physics, 106(8), 2009.

[223] S G Rykovanov, C G R Geddes, J-L Vay, C B Schroeder, E Esarey, and W P Leemans. Quasi-monoenergetic femtosecond photon sources from thomson scattering using laser plasma accelerators and plasma channels. Journal of Physics B: Atomic, Molecular and Optical Physics, 47(23):234013, 2014. 
[224] Ryuta Takashima, Masaru Todoriki, Shuichi Hasegawa, Koshichi Nemoto, and Kazuyuki Kato. Numerical evaluation of nuclide analysis of i129, sr90, and cs137 using bremsstrahlung high energy $\mathrm{x}$ ray generated by ultrashort pulse laser. Journal of Applied Physics, 100(6):-, 2006.

[225] J. Silano, S. Clarke, S. Pozzi, S. Banerjee, D. Haden, G. Golovin, S. Chen, I. Ghebregziabher, C. Liu, C. Petersen, J. Zhang, N. Powers, B. Zhao, K. Brown, J. Mills, and D. Umstadter. Selective activation with all-laser-driven thomson X-rays. In Technologies for Homeland Security (HST), 2013 IEEE International Conference on, pages 429-434, Nov 2013.

[226] T. E. Cowan, A. W. Hunt, T. W. Phillips, S. C. Wilks, M. D. Perry, C. Brown, W. Fountain, S. Hatchett, J. Johnson, M. H. Key, T. Parnell, D. M. Pennington, R. A. Snavely, and Y. Takahashi. Photonuclear fission from high energy electrons from ultraintense laser-solid interactions. Phys. Rev. Lett., 84:903-906, Jan 2000.

[227] Philip M. Walker and James J. Carroll. Ups and downs of nuclear isomers. Physics Today, 58(6), 2005.

[228] Ani Aprahamian and Yang Sun. Nuclear physics: Long live isomer research. Nat Phys, 1(2):81-82, 112005.

[229] S. M. Vinko, O. Ciricosta, B. I. Cho, K. Engelhorn, H.-K. Chung, C. R. D. Brown, T. Burian, J. Chalupsky, R. W. Falcone, C. Graves, V. Hjkov, A. Higginbotham, L. Juha, J. Krzywinski, H. J. Lee, M. Messerschmidt, C. D. Murphy, Y. Ping, A. Scherz, W. Schlotter, S. Toleikis, J. J. Turner, L. Vysin, T. Wang, B. Wu, U. Zastrau, D. Zhu, R. W. Lee, P. A. Heimann, B. Nagler, and J. S. Wark. Nature, 482(Creation and diagnosis of a solid-density plasma with an X-ray free-electron laser):59-62, 2012.

[230] Jonas Gunst, Yuri A. Litvinov, Christoph H. Keitel, and Adriana Pálffy. Dominant secondary nuclear photoexcitation with the x-ray free-electron laser. Phys. Rev. Lett., 112:082501, Feb 2014.

[231] A. Giles Davies, Andrew D. Burnett, Wenhui Fan, Edmund H. Linfield, and John E. Cunningham. Terahertz spectroscopy of explosives and drugs. Materials Today, 11(3):18 - 26, 2008.

[232] R. Appleby and R.N. Anderton. Millimeter-wave and submillimeter-wave imaging for security and surveillance. Proceedings of the IEEE, 95(8):1683-1690, Aug 2007.

[233] R. A. Kaindl, M. A. Carnahan, D. Hagele, R. Lovenich, and D. S. Chemla. Ultrafast terahertz probes of transient conducting and insulating phases in an electron-hole gas. Nature, 423(6941):734-738, 062003.

[234] Tae-In Jeon and D. Grischkowsky. Nature of conduction in doped silicon. Phys. Rev. Lett., 78:1106-1109, Feb 1997. 
[235] K. B. Nordstrom, K. Johnsen, S. J. Allen, A.-P. Jauho, B. Birnir, J. Kono, T. Noda, H. Akiyama, and H. Sakaki. Excitonic dynamical franz-keldysh effect. Phys. Rev. Lett., 81:457-460, Jul 1998.

[236] Fabio Novelli, Daniele Fausti, Francesca Giusti, Fulvio Parmigiani, and Matthias Hoffmann. Mixed regime of light-matter interaction revealed by phase sensitive measurements of the dynamical franz-keldysh effect. Scientific Reports, 3:1227 EP -, 022013.

[237] Rupert Huber, Ben A. Schmid, Y. Ron Shen, Daniel S. Chemla, and Robert A. Kaindl. Stimulated terahertz emission from intraexcitonic transitions in $\mathrm{cu}_{2} \mathrm{O}$. Phys. Rev. Lett., 96:017402, Jan 2006.

[238] J. E. Bailey, T. Nagayama, G. P. Loisel, G. A. Rochau, C. Blancard, J. Colgan, Ph. Cosse, G. Faussurier, C. J. Fontes, F. Gilleron, I. Golovkin, S. B. Hansen, C. A. Iglesias, D. P. Kilcrease, J. J. MacFarlane, R. C. Mancini, S. N. Nahar, C. Orban, J. C. Pain, A. K. Pradhan, M. Sherrill, and B. G. Wilson. A higher-than-predicted measurement of iron opacity at solar interior temperatures. Nature, 517(7532):56-59, 012015.

[239] John Lindl. Development of the indirect drive approach to inertial confinement fusion and the target physics basis for ignition and gain. Physics of Plasmas, 2(11), 1995.

[240] Antoine Rousse, Christian Rischel, and Jean-Claude Gauthier. Femtosecond x-ray crystallography. Rev. Mod. Phys., 73:17-31, Jan 2001.

[241] Christian Rischel, Antoine Rousse, Ingo Uschmann, Pierre-Antoine Albouy, Jean-Paul Geindre, Patrick Audebert, Jean-Claude Gauthier, Eckhart Froster, Jean-Louis Martin, and Andre Antonetti. Femtosecond time-resolved x-ray diffraction from laserheated organic films. Nature, 390(6659):490-492, 121997.

[242] Klaus Sokolowski-Tinten, Christian Blome, Juris Blums, Andrea Cavalleri, Clemens Dietrich, Alexander Tarasevitch, Ingo Uschmann, Eckhard Forster, Martin Kammler, Michael Horn-von Hoegen, and Dietrich von der Linde. Femtosecond x-ray measurement of coherent lattice vibrations near the lindemann stability limit. Nature, 422(6929):287-289, 032003.

[243] Henry N. Chapman, Petra Fromme, Anton Barty, Thomas A. White, Richard A. Kirian, Andrew Aquila, Mark S. Hunter, Joachim Schulz, Daniel P. DePonte, Uwe Weierstall, R. Bruce Doak, Filipe R. N. C. Maia, Andrew V. Martin, Ilme Schlichting, Lukas Lomb, Nicola Coppola, Robert L. Shoeman, Sascha W. Epp, Robert Hartmann, Daniel Rolles, Artem Rudenko, Lutz Foucar, Nils Kimmel, Georg Weidenspointner, Peter Holl, Mengning Liang, Miriam Barthelmess, Carl Caleman, Sebastien Boutet, Michael J. Bogan, Jacek Krzywinski, Christoph Bostedt, Sasa Bajt, Lars Gumprecht, Benedikt Rudek, Benjamin Erk, Carlo Schmidt, Andre Homke, Christian Reich, Daniel Pietschner, Lothar Struder, Gunter Hauser, Hubert Gorke, Joachim Ullrich, Sven Herrmann, Gerhard Schaller, Florian Schopper, Heike Soltau, Kai-Uwe Kuhnel, Marc Messerschmidt, John D. Bozek, Stefan P. Hau-Riege, Matthias Frank, Christina Y. Hampton, Raymond G. Sierra, Dmitri Starodub, Garth J. Williams, Janos Hajdu, 
Nicusor Timneanu, M. Marvin Seibert, Jakob Andreasson, Andrea Rocker, Olof Jonsson, Martin Svenda, Stephan Stern, Karol Nass, Robert Andritschke, Claus-Dieter Schroter, Faton Krasniqi, Mario Bott, Kevin E. Schmidt, Xiaoyu Wang, Ingo Grotjohann, James M. Holton, Thomas R. M. Barends, Richard Neutze, Stefano Marchesini, Raimund Fromme, Sebastian Schorb, Daniela Rupp, Marcus Adolph, Tais Gorkhover, Inger Andersson, Helmut Hirsemann, Guillaume Potdevin, Heinz Graafsma, Bjorn Nilsson, and John C. H. Spence. Femtosecond x-ray protein nanocrystallography. Nature, 470(7332):73-77, 022011.

[244] J. Gaudin, O. Peyrusse, J. Chalupský, M. Toufarová, L. Vyšín, V. Hájková, R. Sobierajski, T. Burian, Sh. Dastjani-Farahani, A. Graf, M. Amati, L. Gregoratti, S. P. Hau-Riege, G. Hoffmann, L. Juha, J. Krzywinski, R. A. London, S. Moeller, H. Sinn, S. Schorb, M. Störmer, Th. Tschentscher, V. Vorlíček, H. Vu, J. Bozek, and C. Bostedt. Amorphous to crystalline phase transition in carbon induced by intense femtosecond x-ray free-electron laser pulses. Phys. Rev. B, 86:024103, Jul 2012.

[245] R. Mankowsky, A. Subedi, M. Forst, S. O. Mariager, M. Chollet, H. T. Lemke, J. S. Robinson, J. M. Glownia, M. P. Minitti, A. Frano, M. Fechner, N. A. Spaldin, T. Loew, B. Keimer, A. Georges, and A. Cavalleri. Nonlinear lattice dynamics as a basis for enhanced superconductivity in yba2cu3o6.5. Nature, 516(7529):71-73, 122014.

[246] P. Beaud, A. Caviezel, S. O. Mariager, L. Rettig, G. Ingold, C. Dornes, S-W. Huang, J. A. Johnson, M. Radovic, T. Huber, T. Kubacka, A. Ferrer, H. T. Lemke, M. Chollet, D. Zhu, J. M. Glownia, M. Sikorski, A. Robert, H. Wadati, M. Nakamura, M. Kawasaki, Y. Tokura, S. L. Johnson, and U. Staub. A time-dependent order parameter for ultrafast photoinduced phase transitions. Nat Mater, 13(10):923-927, 102014.

[247] Wenkai Zhang, Roberto Alonso-Mori, Uwe Bergmann, Christian Bressler, Matthieu Chollet, Andreas Galler, Wojciech Gawelda, Ryan G. Hadt, Robert W. Hartsock, Thomas Kroll, Kasper S. Kjaer, Katharina Kubicek, Henrik T. Lemke, Huiyang W. Liang, Drew A. Meyer, Martin M. Nielsen, Carola Purser, Joseph S. Robinson, Edward I. Solomon, Zheng Sun, Dimosthenis Sokaras, Tim B. van Driel, Gyorgy Vanko, Tsu-Chien Weng, Diling Zhu, and Kelly J. Gaffney. Tracking excited-state charge and spin dynamics in iron coordination complexes. Nature, 509(7500):345-348, 052014.

[248] Yogendra M. Gupta and Surinder M. Sharma. Shocking matter to extreme conditions. Science, 277(5328):909-910, 1997.

[249] M. Koenig, B. Faral, J. M. Boudenne, D. Batani, A. Benuzzi, S. Bossi, C. Rémond, J. P. Perrine, M. Temporal, and S. Atzeni. Relative consistency of equations of state by laser driven shock waves. Phys. Rev. Lett., 74:2260-2263, Mar 1995.

[250] R. F. Smith, J. H. Eggert, R. Jeanloz, T. S. Duffy, D. G. Braun, J. R. Patterson, R. E. Rudd, J. Biener, A. E. Lazicki, A. V. Hamza, J. Wang, T. Braun, L. X. Benedict, P. M. Celliers, and G. W. Collins. Ramp compression of diamond to five terapascals. Nature, 511(7509):330-333, 072014. 
[251] L.M. Barker. Laser interferometry in shock-wave research. Experimental Mechanics, 12(5):209-215, 1972.

[252] F. J. Marshall, P. W. McKenty, J. A. Delettrez, R. Epstein, J. P. Knauer, V. A. Smalyuk, J. A. Frenje, C. K. Li, R. D. Petrasso, F. H. Séguin, and R. C. Mancini. Plasma-density determination from x-ray radiography of laser-driven spherical implosions. Phys. Rev. Lett., 102:185004, May 2009.

[253] D. G. Hicks, N. B. Meezan, E. L. Dewald, A. J. Mackinnon, R. E. Olson, D. A. Callahan, T. Doppner, L. R. Benedetti, D. K. Bradley, P. M. Celliers, D. S. Clark, P. Di Nicola, S. N. Dixit, E. G. Dzenitis, J. E. Eggert, D. R. Farley, J. A. Frenje, S. M. Glenn, S. H. Glenzer, A. V. Hamza, R. F. Heeter, J. P. Holder, N. Izumi, D. H. Kalantar, S. F. Khan, J. L. Kline, J. J. Kroll, G. A. Kyrala, T. Ma, A. G. MacPhee, J. M. McNaney, J. D. Moody, M. J. Moran, B. R. Nathan, A. Nikroo, Y. P. Opachich, R. D. Petrasso, R. R. Prasad, J. E. Ralph, H. F. Robey, H. G. Rinderknecht, J. R. Rygg, J. D. Salmonson, M. B. Schneider, N. Simanovskaia, B. K. Spears, R. Tommasini, K. Widmann, A. B. Zylstra, G. W. Collins, O. L. Landen, J. D. Kilkenny, W. W. Hsing, B. J. MacGowan, L. J. Atherton, and M. J. Edwards. Implosion dynamics measurements at the national ignition facility. Physics of Plasmas, 19(12), 2012.

[254] J. R. Rygg, O. S. Jones, J. E. Field, M. A. Barrios, L. R. Benedetti, G. W. Collins, D. C. Eder, M. J. Edwards, J. L. Kline, J. J. Kroll, O. L. Landen, T. Ma, A. Pak, J. L. Peterson, K. Raman, R. P. J. Town, and D. K. Bradley. 2d x-ray radiography of imploding capsules at the national ignition facility. Phys. Rev. Lett., 112:195001, May 2014.

[255] S. N. Luo, B. J. Jensen, D. E. Hooks, K. Fezzaa, K. J. Ramos, J. D. Yeager, K. Kwiatkowski, and T. Shimada. Gas gun shock experiments with single-pulse Xray phase contrast imaging and diffraction at the advanced photon source. Review of Scientific Instruments, 83(7):-, 2012.

[256] Andreas Schropp, Robert Hoppe, Vivienne Meier, Jens Patommel, Frank Seiboth, Yuan Ping, Damien G. Hicks, Martha A. Beckwith, Gilbert W. Collins, Andrew Higginbotham, Justin S. Wark, Hae Ja Lee, Bob Nagler, Eric C. Galtier, Brice Arnold, Ulf Zastrau, Jerome B. Hastings, and Christian G. Schroer. Imaging shock waves in diamond with both high temporal and spatial resolution at an xfel. Scientific Reports, 5:11089 EP -, 062015.

[257] C. W. Siders, A. Cavalleri, K. Sokolowski-Tinten, Cs. Tth, T. Guo, M. Kammler, M. Horn von Hoegen, K. R. Wilson, D. von der Linde, and C. P. J. Barty. Detection of nonthermal melting by ultrafast x-ray diffraction. Science, 286(5443):1340-1342, 1999.

[258] A. Rousse, C. Rischel, S. Fourmaux, I. Uschmann, S. Sebban, G. Grillon, Ph. Balcou, E. Forster, J. P. Geindre, P. Audebert, J. C. Gauthier, and D. Hulin. Non-thermal melting in semiconductors measured at femtosecond resolution. Nature, 410(6824):6568, 032001. 
[259] A. Levy, P. Audebert, R. Shepherd, J. Dunn, M. Cammarata, O. Ciricosta, F. Deneuville, F. Dorchies, M. Fajardo, C. Fourment, D. Fritz, J. Fuchs, J. Gaudin, M. Gauthier, A. Graf, H. J. Lee, H. Lemke, B. Nagler, J. Park, O. Peyrusse, A. B. Steel, S. M. Vinko, J. S. Wark, G. O. Williams, D. Zhu, and R. W. Lee. The creation of large-volume, gradient-free warm dense matter with an x-ray free-electron laser. Physics of Plasmas, 22(3):-, 2015.

[260] O. Ciricosta, S. M. Vinko, H.-K. Chung, B.-I. Cho, C. R. D. Brown, T. Burian, J. Chalupský, K. Engelhorn, R. W. Falcone, C. Graves, V. Hájková, A. Higginbotham, L. Juha, J. Krzywinski, H. J. Lee, M. Messerschmidt, C. D. Murphy, Y. Ping, D. S. Rackstraw, A. Scherz, W. Schlotter, S. Toleikis, J. J. Turner, L. Vysin, T. Wang, B. Wu, U. Zastrau, D. Zhu, R. W. Lee, P. Heimann, B. Nagler, and J. S. Wark. Direct measurements of the ionization potential depression in a dense plasma. Phys. Rev. Lett., 109:065002, Aug 2012.

[261] B. I. Cho, K. Engelhorn, S. M. Vinko, H.-K. Chung, O. Ciricosta, D. S. Rackstraw, R. W. Falcone, C. R. D. Brown, T. Burian, J. Chalupský, C. Graves, V. Hájková, A. Higginbotham, L. Juha, J. Krzywinski, H. J. Lee, M. Messersmidt, C. Murphy, Y. Ping, N. Rohringer, A. Scherz, W. Schlotter, S. Toleikis, J. J. Turner, L. Vysin, T. Wang, B. Wu, U. Zastrau, D. Zhu, R. W. Lee, B. Nagler, J. S. Wark, and P. A. Heimann. Resonant k $\alpha$ spectroscopy of solid-density aluminum plasmas. Phys. Rev. Lett., 109:245003, Dec 2012.

[262] S. P. Hau-Riege, A. Graf, T. Döppner, R. A. London, J. Krzywinski, C. Fortmann, S. H. Glenzer, M. Frank, K. Sokolowski-Tinten, M. Messerschmidt, C. Bostedt, S. Schorb, J. A. Bradley, A. Lutman, D. Rolles, A. Rudenko, and B. Rudek. Ultrafast transitions from solid to liquid and plasma states of graphite induced by x-ray free-electron laser pulses. Phys. Rev. Lett., 108:217402, May 2012.

[263] Melanie Saes, Christian Bressler, Rafael Abela, Daniel Grolimund, Steven L. Johnson, Philip A. Heinmann, and Majed Chergui. Observing photochemical transients by ultrafast x-ray absorption spectroscopy. Phys. Rev. Lett., 90:047403, 2003.

[264] A. Cavalleri, M. Rini, H. H. W. Chong, S. Fourmaux, T. E. Glover, P. A. Heinmann, J. C. Kieffer, and R. W. Schoenlein. Band-selective measurements of electron dynamics in vo2 using femtosecond near-edge x-ray absorption. Phys. Rev. Lett., 95:067405, 2005.

[265] Y. Ping, F. Coppari, D. G. Hicks, B. Yaakobi, D. E. Fratanduono, S. Hamel, J. H. Eggert, J. R. Rygg, R. F. Smith, D. C. Swift, D. G. Braun, T. R. Boehly, and G. W. Collins. Solid iron compressed up to 560 gpa. Phys. Rev. Lett., 111:065501, 2013.

[266] A. Levy, F. Dorchies, A. Benuzzi-Mounaix, A. Ravasio, F. Festa, V. Recoules, O. Peyrusse, N. Amadou, E. Brambrink, T. Hall, M. Koenig, and S. Mazevet. Xray diagnosis of the pressure induced mott nonmetal-metal transition. Phys. Rev. Lett., 108:055002, 2012. 
[267] A. Benuzzi-Mounaix, F. Dorchies, V. Recoules, F. Festa, O. Peyrusse, A. Levy, A. Ravasio, T. Hall, M. Koenig, N. Amadou, E. Bambrink, and S. Mazevet. Electronic structure investigation of highly compressed aluminum with k-edge absorption spectroscopy. Phys. Rev. Lett., 107:165006, 2011.

[268] F. Dorchies, A. Levy, C. Goyon, P. Combis, D. Descamps, C. Fourment, M. Harmand, S. Hulin, P. M. Leguay, S. Petit, O. Peyrusse, and J. J. Santost. Unraveling the solid-liquid-vapor phase transition dynamics at the atomic level with ultrafast x-ray absorption near-edge spectroscopy. Phys. Rev. Lett., 107:245006, 2011.

[269] F. Dorchies, F. Festa, V. Recoules, O. Peyrusse, A. Benuzzi-Mounaix, E. Brambrink, A. Levy, A. Ravasio, M. Koenig, T. Hall, and S. Mazevet. X-ray absorption $k$ edge as a diagnostic of the electronic temperature in warm dense aluminum. Phys. Rev. B, 92:085117, Aug 2015.

[270] B. I. Cho, K. Engelhorn, A. A. Correa, T. Ogitsu, C. P. Weber, H. J. Lee, J. Feng, P. A. Ni, Y. Ping, A. J. Nelson, D. Prendergast, R. W. Lee, R. W. Falcone, and P. A. Heimann. Electronic structure of warm dense copper studied by ultrafast x-ray absorption spectroscopy. Phys. Rev. Lett., 106:167601, 2011.

[271] K. Engelhorn, V. Recoules, B. I. Cho, B. Barbrel, S. Mazevet, D. M. Krol, R. W. Falcone, and P. A. Heimann. Electronic structure of warm dense silicon dioxide. Phys. Rev. B, 91:214305, Jun 2015.

[272] J. Gaudin, C. Fourment, B. I. Cho, K. Engelhorn, E. Galtier, M. Harmand, P. M. Leguay, H. J. Lee, B. Nagler, M. Nakatsutsumi, C. Ozkan, M. Störmer, S. Toleikis, Th Tschentscher, P. A. Heimann, and F. Dorchies. Towards simultaneous measurements of electronic and structural properties in ultra-fast x-ray free electron laser absorption spectroscopy experiments. Scientific Reports, 4:4724 EP -, 042014.

[273] M. Harmand, A. Ravasio, S. Mazevet, J. Bouchet, A. Denoeud, F. Dorchies, Y. Feng, C. Fourment, E. Galtier, J. Gaudin, F. Guyot, R. Kodama, M. Koenig, H. J. Lee, K. Miyanishi, G. Morard, R. Musella, B. Nagler, M. Nakatsutsumi, N. Ozaki, V. Recoules, S. Toleikis, T. Vinci, U. Zastrau, D. Zhu, and A. Benuzzi-Mounaix. X-ray absorption spectroscopy of iron at multimegabar pressures in laser shock experiments. Phys. Rev. B, 92:024108, Jul 2015.

[274] S. J. Davidson, J. M. Foster, C. C. Smith, K. A. Warburton, and S. J. Rose. Investigation of the opacity of hot, dense aluminum in the region of its k edge. Appl. Phys. Lett., 52:847, 2008.

[275] T. Blenski, G. Loisel, M. Poirier, F. Thais, P. Arnault, T. Caillaud, J. Fariaut, F. Gilleron, J.-C. Pain, Q. Porcherot, C. Reverdin, V. Silvert, B. Villette, S. BastianiCeccotti, S. Turck-Chieze, W. Foelsner, and F. de Gaufridy de Dortan. Opacity of iron, nickel, and copper plasmas in the x-ray wavelength range: Theoretical interpretation of 2p 3d absorption spectra. Phys. Rev. E, 84:036407, 2011. 
[276] Haruhiko Dekura, Taku Tsuchiya, Yasuhiro Kuwayama, and Jun Tsuchiya. Theoretical and experimental evidence for a new post-cotunnite phase of titanium dioxide with significant optical absorption. Phys. Rev. Lett., 107:045701, Jul 2011.

[277] J. C. Adam, A. Héron, G. Laval, and P. Mora. Opacity of an underdense plasma slab due to the parametric instabilities of an ultraintense laser pulse. Phys. Rev. Lett., 84:3598-3601, Apr 2000.

[278] Shinsuke Fujioka, Hiroaki Nishimura, Katsunobu Nishihara, Akira Sasaki, Atsushi Sunahara, Tomoharu Okuno, Nobuyoshi Ueda, Tsuyoshi Ando, Yezheng Tao, Yoshinori Shimada, Kazuhisa Hashimoto, Michiteru Yamaura, Keisuke Shigemori, Mitsuo Nakai, Keiji Nagai, Takayoshi Norimatsu, Takeshi Nishikawa, Noriaki Miyanaga, Yasukazu Izawa, and Kunioki Mima. Opacity effect on extreme ultraviolet radiation from laser-produced tin plasmas. Phys. Rev. Lett., 95:235004, Nov 2005.

[279] O. S. Jones, S. H. Glenzer, L. J. Suter, R. E. Turner, K. M. Campbell, E. L. Dewald, B. A. Hammel, J. H. Hammer, R. L. Kauffman, O. L. Landen, M. D. Rosen, R. J. Wallace, and F. A. Weber. Measurement of the absolute hohlraum-wall albedo under ignition foot drive conditions. Phys. Rev. Lett., 93:065002, Aug 2004.

[280] J. Rosato. Coherence effects on photon absorption in optically thick plasmas. Phys. Rev. Lett., 107:205001, Nov 2011.

[281] D.J. Hoarty, J.W.O. Harris, P. Graham, S.J. Davidson, S.F. James, B.J.B. Crowley, E.L. Clark, C.C. Smith, and L. Upcraft. Measurements of niobium absorption spectra in plasmas with nearly full m-shell configurations. High Energy Density Physics, $3(34): 325-334,2007$.

[282] M. H. Edwards, D. Whittaker, P. Mistry, N. Booth, G. J. Pert, G. J. Tallents, B. Rus, T. Mocek, M. Koslová, C. McKenna, A. Delserieys, C. L. S. Lewis, M. Notley, and D. Neely. Opacity measurements of a hot iron plasma using an x-ray laser. Phys. Rev. Lett., 97:035001, Jul 2006.

[283] J Edwards and S J Rose. Ionization time scales in hot dense plasma. Journal of Physics B: Atomic, Molecular and Optical Physics, 26(16):L523, 1993.

[284] A. L. Kritcher, P. Neumayer, J. Castor, T. Doppner, R. W. Falcone, O. L. Landen, H. J. Lee, R. W. Lee, E. C. Morse, A. Ng, S. Pollaine, D. Price, and S. H. Glenzer. Ultrafast x-ray thomson scattering of shock-compressed matter. Science, 322:69-71, 2008.

[285] M.A. Barrios, K.B. Fournier, S.P. Regan, O. Landen, M. May, Y.P. Opachich, K. Widmann, D.K. Bradley, and G.W. Collins. Backlighter development at the national ignition facility (nif): Zinc to zirconium. High Energy Density Physics, 9(3):626 - 634, 2013. 
[286] F Albert, A G R Thomas, S P D Mangles, S Banerjee, S Corde, A Flacco, M Litos, D Neely, J Vieira, Z Najmudin, R Bingham, C Joshi, and T Katsouleas. Laser wakefield accelerator based light sources: potential applications and requirements. Plasma Physics and Controlled Fusion, 56(8):084015, 2014.

[287] Jeffrey A. Koch, Otto L. Landen, Bernard J. Kozioziemski, Nobuhiko Izumi, Eduard L. Dewald, Jay D. Salmonson, and Bruce A. Hammel. Refraction-enhanced x-ray radiography for inertial confinement fusion and laser-produced plasma applications. Journal of Applied Physics, 105(11), 2009.

[288] M. Z. Mo, Z. Chen, S. Fourmaux, A. Saraf, K. Otani, J. C. Kieffer, Y. Y. Tsui, A. $\mathrm{Ng}$, and R. Fedosejevs. Laser wakefield generated x-ray probe for femtosecond time-resolved measurements of ionization states of warm dense aluminum. Review of Scientific Instruments, 84(12), 2013.

[289] S. H. Glenzer and R. Redmer. X-ray thomson scattering in high energy density plasmas. Rev. Mod. Phys, 81:1625-1663, 2009.

[290] A. J. Comley, B. R. Maddox, R. E. Rudd, S. T. Prisbrey, J. A. Hawreliak, D. A. Orlikowski, S. C. Peterson, J. H. Satcher, A. J. Elsholz, H.-S. Park, B. A. Remington, N. Bazin, J. M. Foster, P. Graham, N. Park, P. A. Rosen, S. R. Rothman, A. Higginbotham, M. Suggit, and J. S. Wark. Strength of shock-loaded single-crystal tantalum [100] determined using In Situ broadband x-ray laue diffraction. Phys. Rev. Lett., 110:115501, Mar 2013.

[291] B. R. Maddox, H. S. Park, B. A. Remington, N. Izumi, S. Chen, C. Chen, G. Kimminau, Z. Ali, M. J. Haugh, and Q. Ma. High-energy x-ray backlighter spectrum measurements using calibrated image plates. Rev. Sc. Instr., 82:023111, 2011.

[292] Matthew Suggit, Giles Kimminau, James Hawreliak, Bruce Remington, Nigel Park, and Justin Wark. Nanosecond x-ray laue diffraction apparatus suitable for laser shock compression experiments. Review of Scientific Instruments, 81(8), 2010.

[293] Christoph Bostedt, Sébastien Boutet, David M. Fritz, Zhirong Huang, Hae Ja Lee, Henrik T. Lemke, Aymeric Robert, William F. Schlotter, Joshua J. Turner, and Garth J. Williams. Linac coherent light source: The first five years. Rev. Mod. Phys., 88:015007, Mar 2016.

[294] N. Rohringer, D. Ryan, R.A. London, M. Purvis, F. Albert, J. Dunn, J.D. Bozek, C. Bostedt, A. Graf, R. Hill, S.P. Hau-Riege, and J.J. Rocca. Nature, 481:488-491, 2012.

[295] Wei Liu, Daniel Wacker, Cornelius Gati, Gye Won Han, Daniel James, Dingjie Wang, Garrett Nelson, Uwe Weierstall, Vsevolod Katritch, Anton Barty, Nadia A. Zatsepin, Dianfan Li, Marc Messerschmidt, Sbastien Boutet, Garth J. Williams, Jason E. Koglin, M. Marvin Seibert, Chong Wang, Syed T. A. Shah, Shibom Basu, Raimund Fromme, Christopher Kupitz, Kimberley N. Rendek, Ingo Grotjohann, Petra Fromme, Richard A. Kirian, Kenneth R. Beyerlein, Thomas A. White, Henry N. Chapman, 
Martin Caffrey, John C. H. Spence, Raymond C. Stevens, and Vadim Cherezov. Serial femtosecond crystallography of g proteincoupled receptors. Science, 342(6165):1521$1524,2013$.

[296] Thomas R. M. Barends, Lutz Foucar, Sabine Botha, R. Bruce Doak, Robert L. Shoeman, Karol Nass, Jason E. Koglin, Garth J. Williams, Sebastien Boutet, Marc Messerschmidt, and Ilme Schlichting. Nature.

[297] D. Milathianaki, S. Boutet, G. J. Williams, A. Higginbotham, D. Ratner, A. E. Gleason, M. Messerschmidt, M. M. Seibert, D. C. Swift, P. Hering, J. Robinson, W. E. White, and J. S. Wark. Femtosecond visualization of lattice dynamics in shockcompressed matter. Science, 342(6155):220-223, 2013.

[298] M. Dell'Angela, T. Anniyev, M. Beye, R. Coffee, A. Fhlisch, J. Gladh, T. Katayama, S. Kaya, O. Krupin, J. LaRue, A. Mgelhj, D. Nordlund, J. K. Nrskov, H. berg, H. Ogasawara, H. strm, L. G. M. Pettersson, W. F. Schlotter, J. A. Sellberg, F. Sorgenfrei, J. J. Turner, M. Wolf, W. Wurth, and A. Nilsson. Real-time observation of surface bond breaking with an x-ray laser. Science, 339(6125):1302-1305, 2013.

[299] Benjamin S. Williams. Terahertz quantum-cascade lasers. Nat Photon, 1(9):517-525, 092007.

[300] International committee on ultra high intensity lasers. http://www.icuil.org/ activities/facilities.html.

[301] Gerard Mourou and Toshiki Tajima. More intense, shorter pulses. Science, 331:41, 2011. 